\title{
ELLIPTIC THERMAL CORRELATION FUNCTIONS AND MODULAR FORMS IN A GLOBALLY CONFORMAL INVARIANT QFT*
}

\author{
NIKOLAY M. NIKOLOV ${ }^{\dagger}$ and IVAN T. TODOROV $\ddagger$ \\ Institute for Nuclear Research and Nuclear Energy, \\ Tsarigradsko Chaussee 72, BG-1784 Sofia, Bulgaria \\ and Institut für Theoretische Physik, Universität Göttingen, \\ Friedrich-Hund-Platz 1, D-37077 Göttingen, Germany
}

October 17, 2018

\begin{abstract}
Global conformal invariance (GCI) of quantum field theory (QFT) in two and higher space-time dimensions implies the Huygens' principle, and hence, rationality of correlation functions of observable fields [29]. The conformal Hamiltonian $H$ has discrete spectrum assumed here to be finitely degenerate. We then prove that thermal expectation values of field products on compactified Minkowski space can be represented as finite linear combinations of basic (doubly periodic) elliptic functions in the conformal time variables (of periods 1 and $\tau$ ) whose coefficients are, in general, formal power series in $q^{\frac{1}{2}}=e^{i \pi \tau}$ involving spherical functions of the "space-like" fields' arguments. As a corollary, if the resulting expansions converge to meromorphic functions, then the finite temperature correlation functions are elliptic. Thermal 2-point functions of free fields are computed and shown to display these features. We also study modular transformation properties of Gibbs energy mean values with respect to the (complex) inverse temperature $\tau\left(\operatorname{Im} \tau=\frac{\beta}{2 \pi}>0\right)$. The results are used to obtain the thermodynamic limit of thermal energy densities and correlation functions.
\end{abstract}

Keywords: 4-dimensional conformal field theory, thermal correlation functions, elliptic functions, modular forms

Mathematics Subject Classification 2000: 81T40, 81R10, 81T10

\section{Contents}

1 Introduction 2

1.1 Conformal invariance in $\mathrm{QFT} \ldots \ldots \ldots \ldots$

1.2 Why thermal correlation functions should be elliptic in the conformal time differences? . . . . . . . . . . . . . . . 4

1.3 Basic (anti)periodic functions. Content of the paper $\ldots \ldots \ldots 7$

* to appear in Rev. Math. Phys.

†mitov@inrne.bas.bg, nikolov@theorie.physik.uni-goe.de

†todorov@inrne.bas.bg, itodorov@theorie.physik.uni-goe.de 


\section{Globally Conformal Invariant QFT on Compactified Minkowski} Space

2.1 Affine coordinate systems on compactified Minkowski space . . . . . 9

2.2 Wightman axioms for conformal field theory in the analytic picture . 12

3 GCI Correlation Functions as Meromorphic Functions $\quad 16$

3.1 Rationality of the vacuum correlation functions . . . . . . . . . . 16

3.2 Ellipticity of the finite temperature correlation functions . . . . . . . 21

4 Free Field Models $\quad 26$

4.1 General properties of thermal correlation functions of free fields . . . 26

4.2 Free scalar fields . . . . . . . . . . . . . . . . . . . . 29

4.2.1 Canonical free massless field in even space time dimension $D \quad 31$

4.2.2 Subcanonical field of dimension $d=1$ for $D=6 \ldots 33$

4.3 The Weyl field . . . . . . . . . . . . . . . . . . . . . . 33

4.4 The Maxwell free field . . . . . . . . . . . . . . . . . . . . . 38

5 The Thermodynamic Limit $\quad 40$

5.1 Compactified Minkowski space as a "finite box" approximation . . . 40

5.2 Infinite volume limit of the thermal correlation functions . . . . . . . 43

6 Concluding Remarks $\quad 46$

$\begin{array}{lr}\text { Appendix A. Basic Elliptic Functions } & 47\end{array}$

Appendix B. Proof of Proposition 3.4 52

\section{Introduction}

The modular group $S L(2, \mathbb{Z})(=: \Gamma(1))$ arises as the symmetry group of an oriented 2-dimensional lattice. Usually - including our case - this is the period lattice of an elliptic function. The factor group $P S L(2, \mathbb{Z})=S L(2, \mathbb{Z}) /_{\mathbb{Z}_{2}}$ of $S L(2, \mathbb{Z})$ with respect to its 2 -element centre $\mathbb{Z}_{2} \equiv \mathbb{Z} / 2 \mathbb{Z}$ acts faithfully by fractional linear transformations on the upper half-plane

$$
\mathfrak{H}:=\{\tau \in \mathbb{C}: \operatorname{Im} \tau>0\}, \quad g(\tau)=\frac{a \tau+b}{c \tau+d} \text { for } g=\left(\begin{array}{ll}
a & b \\
c & d
\end{array}\right) \in S L(2, \mathbb{Z}) .
$$

The modular inversion, the involutive $S$-transformation of $\mathfrak{H}$,

$$
S=\left(\begin{array}{rr}
0 & -1 \\
1 & 0
\end{array}\right): \tau \rightarrow-\frac{1}{\tau} \quad(\operatorname{Im} \tau>0),
$$

which relates high and low temperature behaviour, is the oldest and best studied example of a duality transformation [21] (for a recent reference in the context of elliptic functions that provides a historical review going back to 19th century work - see [10]). It naturally appears in the study of finite temperature correlation 
functions in a conformally invariant field theory (CFT). The case of 2-dimensional (2D) CFT has been thoroughly studied from the point of view of vertex algebras in [38]. The present paper builds on the observation that this analysis can be extended in a straightforward manner to the recently developed GCI QFT (see [29] [27] [28]).

\subsection{Conformal invariance in $Q F T$}

The conformal group $\mathcal{C}$ acts on Minkowski space $M$ by local diffeomorphisms which preserve the conformal class of the metric form, - i.e. multiply it by a positive factor. Unlike the Poincaré group, $\mathcal{C}$ acts, in general, by nonlinear transformations on $M$ which may have singularities on a cone (or on a hyperplane). Furthermore, $\mathcal{C}$ has an infinite sheeted universal cover. In view of these peculiarities, there exist different notions of conformal invariance in QFT. In order to make clear the concept of GCI QFT we shall briefly discuss these notions in the framework of axiomatic QFT ([34]).

The weakest condition of conformal invariance in QFT is the infinitesimal conformal invariance of the Wightman functions. This yields a system of first order differential equations for each Wightman function (displayed in Sect. 2.2). According to the Bargman-Hall-Wightman theorem (see [18] Sect. IV.4-5) the Wightman functions are boundary values of analytic functions, holomorphic in a complex domain, the so called symmetrized extended tube, which contains all non-coinciding euclidean arguments. As a result, the same (complexified) system of differential equations is satisfied by these analytic functions and hence by their (real analytic) euclidean restrictions that define the so called non-coinciding Schwinger (or Euclidean Green) functions which are, therefore, invariant under (Euclidean) infinitesimal conformal transformations.

We thus see that the conditions of infinitesimal conformal invariance for each element in the hierarchy of functions - Wightman distributions, their analytic continuations, and the Schwinger functions - are equivalent. The global, i.e. group, version of conformal invariance is more subtle.

We recall that there exist conformal compactifications of both Minkowski and Euclidean space, such that the local actions of the corresponding conformal groups can be extended to everywhere defined ones. The conformally compactified Euclidean space is just the (simply connected) sphere $\mathbb{S}^{D}$. It follows that the infinitesimal Euclidean conformal invariance is equivalent to invariance under the Euclidean conformal group. Compactified Minkowski space $\bar{M}$, on the other hand, is isomorphic to $\mathbb{S}^{1} \times \mathbb{S}^{D-1} / \mathbb{Z}_{2}$ so that it has an infinite sheeted universal cover, $\widetilde{M}$. One can only integrate, in general, the conditions of infinitesimal invariance to invariance on this infinite sheeted cover, the finite conformal transformations on $\widetilde{M}$ becoming multivalued if projected on $\bar{M}$. Assuming euclidean conformal invariance Lüscher and Mack [23] have thus established invariance of the QFT continued to $\widetilde{M}$ under the infinite sheeted covering of the Minkowski space conformal group and called it "global conformal invariance" on the universal covering space. The above anal- 
ysis shows that it is, in fact, equivalent to infinitesimal conformal invariance in Minkowski space.

By contrast, the GCI condition introduced in $[29]^{1}$, invariance under finite conformal transformations $g$ in Minkowski space $M$ (whenever both $x$ and $g x$ are in $M$ ) is stronger since it allows to continue the Wightman functions to invariant distributions on $\bar{M}$. Combined with locality GCI on $M$ implies the Huygens' principle - the vanishing of field (anti)commutators for non-isotropic separations-since it allows to transform space-like into time like intervals. Under Wightman axioms the Huygens principle is equivalent to strong locality (i.e. the algebraic condition (2.22)). (Note that only in even dimensional space-time the canonical free massless fields and the stress-energy tensor satisfy the Huygens principle.) Strong locality and energy positivity imply the rationality of Wightman functions (cf. Theorem 3.3 below), thus excluding non-integer anomalous field dimensions. In the case of 2D CFT the GCI incorporates the notion of chiral algebra which has served as a starting point for developing the important mathematical concept of a vertex algebra - see [2,3], [19], [11] and further references in the latter two books. Moreover, it also includes non-chiral $2 \mathrm{D}$ fields with rational correlation functions. (The simplest example is given by the energy density of conformal weight $\left(\frac{1}{2}, \frac{1}{2}\right)$ in the vacuum sector of the critical Ising model.) The primary fields, which may well have anomalous dimensions and nontrivial braiding properties (as the magnetization field in the Ising model), appear in this framework as intertwiners between the vacuum and other positive energy representations of the GCI algebra. It is then expected that only the 4-dimensional counterpart of such intertwining primary fields may display anomalous time-like braiding relations of the type discussed in [31].

\subsection{Why thermal correlation functions should be elliptic in the conformal time differences?}

The main idea is simple to explain. A GCI QFT lives on compactified Minkowski space $\bar{M}$ of dimension $D$ which has a natural complex vector parametrization:

$$
\bar{M}=\mathbb{S}^{1} \times \mathbb{S}^{D-1} / \mathbb{Z}_{2}=\left\{z_{\alpha}=e^{2 \pi i \zeta} u_{\alpha}: \zeta \in \mathbb{R}, u^{2}:=\boldsymbol{u}^{2}+u_{D}^{2}=1, u \in \mathbb{R}^{D}\right\},
$$

$\zeta$ being the conformal time variable. The coordinates $z$ in Eq. (1.3) are obtained by a complex conformal transformation introduced in Sect. 2.1 (Eq. (2.3)) of the Cartesian coordinates of Minkowski space generalizing the Cayley transform (inverse stereographic projection) of the chiral (i. e. 1-dimensional) case. They have been first introduced for $D=4$ in [35] using the Cayley $(u(2) \rightarrow U(2))$ compactification map, and were generalized to arbitrary $D$ in [30]; the reader will find a geometric introduction to this and more general systems of charts in [26]. The use

\footnotetext{
${ }^{1}$ A special case of such a condition - in the context of (generalized)free fields - has been displayed earlier in [17]; see also [13] where a condition of this type is discussed in the framework of 2D CFT and [16] for a retrospective view on the subject.
} 
of Euclidean metric in (1.3) does not mean, of course, that we are working within the Euclidean picture of QFT at this point. We recall that the Euclidean rotation group $S O(D)$ is a subgroup of the Minkowski space conformal group $S O(D, 2)$ and that (1.3) (involving the Euclidean unit sphere $\mathbb{S}^{D-1}$ ) does indeed represent compactifed Minkowski space. (This is made clear in Sect. 2.1 by exhibiting its relation to the Dirac projective quadric.)

Transforming the fields in these coordinates we obtain an equivalent representation of the GCI local fields on $\bar{M}$ called (analytic or) $z$-picture. Since the transformation is conformal the vacuum correlation (Wightman) functions do not change their form. For example, the $z$-picture scalar field $\phi(z)$ of (integer) dimension $d$ has rational correlation functions like

$$
\left\langle 0\left|\phi\left(z_{1}\right) \phi\left(z_{2}\right)\right| 0\right\rangle=\left(z_{12}^{2}\right)^{-d}, \quad z_{12}=z_{1}-z_{2},
$$

invariant under $D$-dimensional inhomogeneous complex rotation group. Let us note that we will treat the fields as formal power series in $z$ and $\frac{1}{z^{2}}$ which is shown in [26] to be completely equivalent to the Wightman approach (with GCI) which treats local fields as operator valued distributions.

The conformal Hamiltonian $H$, with respect to which we will consider the thermal correlation functions, gives rise to a multiplication of $z$ by a phase factor (and hence, to a translation of $\zeta$ in Eq. (1.3)). This suggests introducing a real compact picture field $\phi(\zeta, u)$ related to $\phi(z)$ by

$$
\phi(\zeta, u)=e^{2 \pi i d \zeta} \phi\left(e^{2 \pi i \zeta} u\right) .
$$

Then $H$ acts on it by (infinitesimal) translation in $\zeta$ :

$$
e^{2 \pi i t H} \phi(\zeta, u) e^{-2 \pi i t H}=\phi(\zeta+t, u) .
$$

Since $H$ has an integer or half-integer spectrum in the vacuum sector state space it follows that:

$$
\phi(\zeta+1, u)=(-1)^{2 d} \phi(\zeta, u),
$$

i. e., the conformal time evolution is periodic or anti-periodic with period 1 in the compact picture, so that the vacuum and the thermal correlation functions will be also (anti-) periodic.

The second period $\tau$ comes from the statistical quantum physics: there it is pure imaginary and proportional to the inverse absolute temperature. More precisely, for any (real) Bose field $\phi$ with an invariant dense domain $\mathcal{D}$ (common for all fields' and actually coinciding with the finite energy space spanned by eigenvectors of $H$ - see Proposition 3.2), we are going to construct the partition function

$$
Z(\tau)=\operatorname{tr}_{\mathcal{D}}\left(q^{H}\right), \quad q=e^{2 \pi i \tau}, \quad \operatorname{Im} \tau>0 \quad(|q|<1)
$$

and the Gibbs correlation functions

$$
\left\langle\phi\left(\zeta_{1}, u_{1}\right) \ldots \phi\left(\zeta_{n}, u_{n}\right)\right\rangle_{q}:=\frac{1}{Z(\tau)} \operatorname{tr}_{\mathcal{D}}\left\{\phi\left(\zeta_{1}, u_{1}\right) \ldots \phi\left(\zeta_{n}, u_{n}\right) q^{H}\right\}
$$


as meromorphic functions in $\tau, \zeta_{k}$ and $u_{k}(k=1, \ldots, n)$ in a suitable domain of $\mathbb{C}^{n D+1}$. Sure, the existence of Gibbs equilibrium states with the above properties requires additional assumptions extending the notion of classical phase space volume. Such extra assumptions are needed in any axiomatic treatment of thermodynamic properties of local $\mathrm{QFT}^{2}$. Our study of GCI QFT is facilitated by the fact that the conformal Hamiltonian $H$ has a (bounded below) discrete spectrum $\left\{\frac{n}{2}: n=0,1, \ldots\right\}$. The partition function (1.8) exists for any inverse temperature $\beta=2 \pi \operatorname{Im} \tau(>0)$ iff the growth of dimension $d\left(\frac{n}{2}\right)$ of the $n$th eigenspace of $H$ is slower than any exponential $e^{\varepsilon n}(\varepsilon>0)$. Moreover, in the GCI QFT it is sufficient to assume that $H$ has just finitely degenerate spectrum to ensure the existence of thermal correlation functions (1.9) (and the partition function (1.8)) as formal power series in $q^{\frac{1}{2}}=e^{i \pi \tau}$ with coefficients which are symmetric rational functions in $\left(e^{\pi i \zeta_{1}}, u_{1}\right), \ldots,\left(e^{\pi i \zeta_{n}}, u_{n}\right)$, as it is shown in Sect. 2. This allows then to extend the heuristic argument, given in [38], which makes it plausible that the Kubo-Martin-Schwinger (KMS) property $[15]^{3}$

$$
\left\langle\phi\left(\zeta_{1}, u_{1}\right) \ldots \phi\left(\zeta_{n}, u_{n}\right)\right\rangle_{q}=\left\langle\phi\left(\zeta_{2}, u_{2}\right) \ldots \phi\left(\zeta_{n}, u_{n}\right) \phi\left(\zeta_{1}+\tau, u_{1}\right)\right\rangle_{q}
$$

implies that the functions (1.9) are doubly periodic meromorphic functions with periods 1 and $\tau$ in $\zeta_{j k}=\zeta_{j}-\zeta_{k}$; in other words, they are elliptic functions. In Sect. 3 we give a rigorous interpretation of this argument thus proving that the finite temperature correlation functions (1.9) has the form of finite linear combinations of basic (series of) elliptic functions in the conformal time variables whose coefficients are, in general, formal power series in $q^{\frac{1}{2}}=e^{i \pi \tau}$ involving spherical functions of the angular fields' arguments $u_{k}$ (Theorem 3.5). ${ }^{4}$ Let us stress that our main result, Theorem 3.5, takes into account the most general purely algebraic properties of the theory only. As noted above additional hypotheses of topological character are necessary in order to guarantee the existence of the thermal expectation values as meromorphic functions. In this case our analysis tells us that these meromorphic functions are automatically elliptic (Corollary 3.6). We shall demonstrate that this is indeed the case for conformally invariant free fields by computing explicitly their Gibbs 2-point functions.

\footnotetext{
${ }^{2}$ For a general discussion of this point within Haag's operator algebra approach - see Sect. V.5 of [14], where Buchholz nuclearity condition [6] [7] is advocated and reviewed.

${ }^{3}$ For a later discussion combining nuclearity, KMS and Lorentz invariance - see [5].

${ }^{4}$ We note that our results are valid in any space-time dimension $D$ which, in particular, for the $D=1$ case, corresponding to the chiral projection of the 2-dimensional CFT, implies that under the assumptions of convergence of all the traces of products of fields' modes (including the partition function (1.8)), the finite temperature correlation functions are convergent to elliptic functions (since then there are no additional angular variables).
} 


\subsection{Basic (anti)periodic functions. Content of the paper}

An elliptic function is characterized by its poles and their residues (in the fundamental domain). The poles of the thermal correlation functions should be the same as the poles of the operator product expansions (OPE): they only appear at mutually isotropic field arguments. In the compact picture the light cone equation factorizes:

$$
0=z_{12}^{2}=\left(e^{2 \pi i \zeta_{1}} u_{1}-e^{2 \pi i \zeta_{2}} u_{2}\right)^{2} \equiv-4 e^{2 \pi i\left(\zeta_{1}+\zeta_{2}\right)} \sin \pi \zeta_{+} \sin \pi \zeta_{-} ;
$$

here we have introduced the variables

$$
\zeta_{ \pm}=\zeta_{12} \pm \alpha, \text { for } u_{1} \cdot u_{2}=\cos 2 \pi \alpha .
$$

Therefore, the basic elliptic functions occurring in the theory depend on the variables of type (1.12) and have poles on the lattice spanned by the periods 1 and $\tau$.

Taking into account the fermionic case the above statements are modified, the periodicity in both periods 1 and $\tau$ being replaced by antiperiodicity. We are thus led to the set $\left\{p_{k}^{\kappa, \lambda}(\zeta, \tau): k=1,2, \ldots, \kappa, \lambda=0,1\right\}$ of basic (elliptic) functions, uniquely characterized by the conditions:

(i) $p_{k}^{\kappa, \lambda}(\zeta, \tau)$ are meromorphic functions in $(\zeta, \tau) \in \mathbb{C} \times \mathfrak{H}$ with exactly one pole at $\zeta=0$ of order $k$ and residue 1 in the domain $\{\alpha \tau+\beta: \alpha, \beta \in[0,1)\} \subset \mathbb{C}$ for all $\tau \in \mathfrak{H}$ and $k=1,2, \ldots$;

(ii) $p_{k+1}^{\kappa, \lambda}(\zeta, \tau)=-\frac{1}{k} \frac{\partial}{\partial \zeta} p_{k}^{\kappa, \lambda}(\zeta, \tau)$ for $k=1,2, \ldots$;

(iii) $p_{k}^{\kappa, \lambda}(\zeta+1, \tau)=(-1)^{\lambda} p_{k}^{\kappa, \lambda}(\zeta, \tau)$ for $k=1,2, \ldots$;

(iv) $p_{k}^{\kappa, \lambda}(\zeta+\tau, \tau)=(-1)^{\kappa} p_{k}^{\kappa, \lambda}(\zeta, \tau)$ for $k+\kappa+\lambda>1$;

(v) $p_{k}^{\kappa, \lambda}(-\zeta, \tau)=(-1)^{k} p_{k}^{\kappa, \lambda}(\zeta, \tau)$ for $k=1,2, \ldots$

Note that for $k=1$ and $\kappa=\lambda=0$ at most one of conditions (iii) and (iv) can be satisfied and we have chosen the first one. This is a natural choice since the periodicity with period 1 in the conformal time is coupled to the periodicity in the angle $\alpha$. It leads to a difference between our $p_{1}^{00}=: p_{1}$ and $p_{2}^{00}=: p_{2}$-functions, and the Weierstrass $\mathfrak{Z}^{-}$and $\wp$-functions (Eqs. (A.6) and (A.5)), respectively, by linear functions in $\zeta$ (see Eqs. (A.11) and (A.12); the Weierstrass functions have the advantage that they have simple modular transformation laws). In Appendix A (see Proposition A.2) we allow for a more general $U(1)$ character replacing $(-1)^{\kappa}$ in condition $(i v): p_{k}^{\kappa, \lambda}(\zeta+\tau, \tau, \mu)=(-1)^{\kappa} e^{-2 \pi i \mu} p_{k}^{\kappa, \lambda}(\zeta, \tau, \mu)$, where the parameter $\mu$ can be interpreted as chemical potential in physical applications (and $p_{k}^{\kappa, \lambda}(\zeta, \tau, 0)=$ $\left.p_{k}^{\kappa, \lambda}(\zeta, \tau)\right)$.

The $n$-point correlation functions (1.9) are elliptic in $\zeta_{j k}=\zeta_{j}-\zeta_{k}$ with poles at $\pm \alpha_{j k}+m+n \tau(n, m \in \mathbb{Z})$, where $\cos 2 \pi \alpha_{j k}=u_{j} \cdot u_{k}$. One cannot expect, however, that they are homogeneous under modular transformations

$$
g(\zeta, \tau)=\left(\frac{\zeta_{j k}}{c \tau+d}, \frac{a \tau+b}{c \tau+d}\right) \quad \text { for } \quad g=\left(\begin{array}{ll}
a & b \\
c & d
\end{array}\right) \in S L(2, \mathbb{Z})
$$


since $\alpha_{j k}$, playing the role of spherical distances for $D>2$, are not invariant under rescaling $\alpha_{j k} \mapsto \frac{\alpha_{j k}}{c \tau+d}$. One can hope to recover modular covariance for their (always well defined, in Wightman theories - see [4]) 1-dimensional restrictions corresponding to

$$
u_{1}=u_{2}=\ldots=u_{n}, \quad \alpha_{j k}=0 .
$$

It turns out that the restricted 2-point function of a $d=1$ free massless scalar field for $D=4$ indeed transforms homogeneously (of degree 2) under the modular transformations (1.13). The corresponding energy mean value in an equilibrium state,

$$
\langle H\rangle_{q}=\frac{1}{Z(\tau)} \operatorname{tr}_{\mathcal{D}}\left(H q^{H}\right)
$$

is a modular form of weight 4 (and level $\Gamma(1)(\equiv S L(2, \mathbb{Z})))$ - after shifting the vacuum energy (Sect. 4.2.1).

The paper is organized as follows.

In Sect. 2.1 we give a concise review of the basic properties of the conformal group and its Lie algebra, and introduce the basic complex parametrization of Minkowski space which we use throughout this paper. It allows us to formulate in Sect. 2.2 an algebraic counterpart of the Wightman axioms in what we call the analytic $(z)$ picture. We sum up the implications of these axioms in Sect. 3.1 where we also give an introduction to the purely algebraic approach to GCI QFT in terms of formal power series. In Sect. 3.2 we obtain the general form of the thermal correlation functions. In Sect. 4 we calculate the finite temperature correlation functions in the (generalized) free field GCI models starting with their relation to the Wightman functions found in Sect. 4.1. The cases of physical free fields in $D=4$ dimensions: the massless scalar, Weyl and electromagnetic fields are considered in Sects. 4.2, 4.3 and 4.4, respectively. We have also studied examples of subcanonical free fields (for $D=4$ and $D=6$ ). The "thermodynamic limit" in which the compactification radius $R$ goes to infinity (so that $\bar{M}$ is restricted to $M$ and time is no longer cyclic) is considered in Sect. 5 where it is shown that the thermal correlation functions have Minkowski space limits. The results are summed up and discussed in Sect. 6. The reader will find our conventions about elliptic functions and modular forms, used in the text, in Appendix A.

\section{Globally Conformal Invariant QFT on Compactified Minkowski Space}

In the GCI QFT the natural choice of the conformal group $\mathcal{C}$ is the connected spinor group $\operatorname{Spin}(D, 2)(\cong \mathcal{C})$. Then the complexified conformal group will be $\mathcal{C}_{\mathbb{C}} \cong$ $\operatorname{Spin}_{\mathbb{C}}(D+2)$. The conformal Lie algebra will be denoted by $c(\cong \operatorname{spin}(D, 2) \cong$ so $(D, 2))$ and its complexification, by $\mathfrak{c}_{\mathbb{C}}$. We begin this section with recalling some basic facts about the conformal group and its action on compactified Minkowski space. 


\subsection{Affine coordinate systems on compactified Minkowski space}

Let $M$ be the $D$-dimensional Minkowski space, with coordinates $x=\left(x^{0}, \boldsymbol{x}=\right.$ $\left.\left(x^{1}, \ldots, x^{D-1}\right)\right) \in \mathbb{R}^{D}$ and Poincaré invariant interval $x_{12}^{2}=\boldsymbol{x}_{12}^{2}-\left(x_{12}^{0}\right)^{2}, x_{12}=$ $x_{1}-x_{2}, \boldsymbol{x}_{12}^{2}=\sum_{j=1}^{D-1}\left(x_{12}^{j}\right)^{2}$. The group of conformal transformations of $M$ is defined as the group of (local) diffeomorphisms of $M(\ni x \mapsto y)$ preserving the conformal class of the infinitesimal metric $d x^{2}\left(\equiv d x^{\mu} d x_{\mu}\right)$, i.e., mapping $d x^{2}$ on $d y^{2}=\omega^{-2}(x)$ $d x^{2}(\omega(x) \neq 0)$. It is finite $\left(\frac{(D+1)(D+2)}{2}\right)$ dimensional for $D \geqslant 3$, due to the Liouville theorem, and is generated by:

- the Poincaré translations $e^{i a \cdot P}(x)\left(\equiv e^{i a^{\mu} P_{\mu}}(x)\right)=x+a$ (for $\left.x, a \in M\right)$,

- the Lorentz transformations $e^{t \Omega_{\mu \nu}}, 0 \leqslant \mu<\nu \leqslant D-1\left(\Omega_{\nu \mu}=-\Omega_{\mu \nu}\right)$,

- the dilations $x \mapsto \rho x, \rho>0$

- and the special conformal transformations $e^{i a \cdot K}(x)=\frac{x+x^{2} a}{1+2 a \cdot x+a^{2} x^{2}}$ (which has obvious singularities).

The corresponding Lie algebra is isomorphic to the Lie algebra of the pseudoorthogonal group $S O(D, 2)$. Recalling this isomorphism we introduce the basis of infinitesimal (pseudo) rotations $\Omega_{a b}\left(=-\Omega_{b a}\right)$, where the indices $a$ and $b$ take values $-1,0, \ldots, D$, the underlying orthonormal basis $\vec{e}_{a} \in \mathbb{R}^{D, 2}(a=-1,0,1, \ldots$, $D$ ) satisfying $\vec{e}_{\alpha}^{2}=1=-\vec{e}_{-1}^{2}=-\vec{e}_{0}^{2}, \alpha=1, \ldots, D$ (cf. [29] Appendix A). The generators $\Omega_{a b}$ are characterized by the following nontrivial commutation relations

$$
\begin{gathered}
{\left[\Omega_{a \alpha}, \Omega_{b \alpha}\right]=\Omega_{a b}\left(=\left[\Omega_{\alpha a}, \Omega_{\alpha b}\right]\right) \text { for } \alpha=1, \ldots, D,} \\
{\left[\Omega_{\kappa a}, \Omega_{\kappa b}\right]=-\Omega_{a b} \text { for } \kappa=-1,0}
\end{gathered}
$$

$i P_{\mu}, i K_{\mu}$ and the dilations are expressed in terms of them as:

$$
i P_{\mu}=-\Omega_{-1 \mu}-\Omega_{\mu D}, \quad i K_{\mu}=-\Omega_{-1 \mu}+\Omega_{\mu D}, \quad \rho^{-\Omega_{-1 D}}(x)=\rho x \quad(\rho>0) ;
$$

the Lorentz generators $\Omega_{\mu \nu}$ correspond to $0 \leqslant \mu, \nu \leqslant D-1$. In fact, the group $S O(D, 2)$ itself has an action on $M$ by (rational) conformal transformations. It is straightforward to derive this action using the Klein-Dirac construction of compactified Minkowski space $\bar{M}$ (1.3), realized as the projective quadric of $\mathbb{R}^{D, 2}$ ([9], see also [29] Appendix A for a survey adapted to our present purposes and notation). The Minkowski space $M$ is mapped into a dense subset (identified with $M$ ) of $\bar{M}$ thus providing an affine chart in $\bar{M}$.

Other affine charts in $\bar{M}$ can be obtained by applying conformal transformations. In particular, the following chart in the complex compactified Minkowski space $\bar{M}_{\mathbb{C}}$ plays a crucial role in the GCI QFT.

Let $M_{\mathbb{C}}:=M+i M$ be the complexified Minkowski space, with coordinates $\mathcal{z}=\left(\mathcal{z}^{0}, \boldsymbol{z}\right)=x+i y \in \mathbb{C}^{D}, \mathcal{z}_{12}^{2} \equiv\left(\mathcal{z}_{1}-\mathcal{Z}_{2}\right)^{2}=\mathcal{z}_{12}^{2}-\left(\mathcal{z}_{12}^{0}\right)^{2}$ being the Poincaré invariant interval and let $E_{\mathbb{C}}$ be the complex Euclidean $D$-dimensional space with 
coordinates $z=\left(\boldsymbol{z}, z_{D}\right) \in \mathbb{C}^{D}$ and Euclidean invariant interval $z_{12}^{2}=\boldsymbol{z}_{12}^{2}+\left(z_{12}^{D}\right)^{2}$, $z_{12}=z_{1}-z_{2}$. The rational complex coordinate transformation (see [35], [30], [26]):

$$
g_{c}: M_{\mathbb{C}}(\ni \mathcal{Z}) \rightarrow E_{\mathbb{C}}(\ni z), \quad z=\frac{\mathcal{Z}}{\omega(\mathcal{Z})}, \quad z_{D}=\frac{1-\mathcal{Z}^{2}}{2 \omega(\mathcal{Z})}, \quad \omega(\mathcal{Z})=\frac{1+\mathcal{Z}^{2}}{2}-i \mathcal{Z}^{0}
$$

is a complex conformal map (with singularities) such that

$$
z_{12}^{2}=\frac{\mathcal{Z}_{12}^{2}}{\omega\left(\mathcal{Z}_{1}\right) \omega\left(\mathcal{Z}_{2}\right)}, \quad d z^{2}\left(=d z^{2}+d z_{D}^{2}\right)=\frac{d \mathcal{Z}^{2}}{\omega(\mathcal{z})^{2}}
$$

The transformation (2.3) is regular on the real Minkowski space $M$ and on the forward tube domain $\mathfrak{T}_{+}=\left\{\mathcal{Z}=x+i y: y^{0}>|\boldsymbol{y}|\right\}$, and maps them on precompact subsets of $E_{\mathbb{C}}$. The closure $\overline{g_{c}(M)}$ of the image of the real Minkowski space $M$ has the form (1.3) (thus being identified with $\bar{M}$ ) and the image $T_{+}$of $\mathfrak{T}_{+}$under $g_{c}$ is

$$
T_{+}:=\left\{z \in \mathbb{C}^{D}:\left|z^{2}\right|<1, z \cdot \bar{z}=\left|z^{1}\right|^{2}+\ldots+\left|z^{D}\right|^{2}<\frac{1}{2}\left(1+\left|z^{2}\right|^{2}\right)\right\}
$$

The conjugation $*: \bar{M}_{\mathbb{C}} \rightarrow \bar{M}_{\mathbb{C}}$ which leaves invariant the real space $\bar{M}$ is represented in the $z$-coordinates as:

$$
z \mapsto z^{*}:=\frac{\bar{z}}{\bar{z}^{2}} \equiv j_{W}\left(R_{D}(\bar{z})\right)
$$

where

$$
R_{\alpha}(z):=\left(z^{1}, \ldots,-z^{\alpha}, \ldots, z^{D}\right), \quad j_{W}(z):=\frac{R_{D}(z)}{z^{2}}
$$

and $j_{W}$ is a $z$-picture analogue of the Weyl reflection.

Let us introduce the complex Lie algebra generators $T_{\alpha}$ and $C_{\alpha}$ for $\alpha=1, \ldots, D$ of the $z$-translations $e^{w \cdot T}(z)=z+w$ and the $z$-special conformal transformations $e^{w \cdot C}(z)=\frac{z+z^{2} w}{1+2 w \cdot z+w^{2} z^{2}}\left(w, z \in \mathbb{C}^{D}\right)$ which are conjugated by $g_{c}$ to the analogous generators $i P_{\mu}$ and $i K_{\mu}$. This new basis of generators is expressed in terms of $\Omega_{a b}$ as:

$$
T_{\alpha}=i \Omega_{0 \alpha}-\Omega_{-1 \alpha}, C_{\alpha}=-i \Omega_{0 \alpha}-\Omega_{-1 \alpha} \text { for } \alpha=1, \ldots, D .
$$

The set of infinitesimal $z$-rotations is again a subset $\left\{\Omega_{\alpha \beta}\right\}$ of $\left\{\Omega_{a b}\right\}$ corresponding to $1 \leqslant \alpha<\beta \leqslant D$ and the conformal generator which gives rise to the dilation (or, in fact, phase) transformation of the $z$-coordinates (to be interpreted as a conformal time translation) is the conformal Hamiltonian

$$
H=i \Omega_{-10}, \quad e^{i t H}(z)=e^{i t} z
$$

$\left(\left[T_{\alpha}, C_{\beta}\right]=2\left(\delta_{\alpha \beta} H-\Omega_{\alpha \beta}\right),\left[H, C_{\alpha}\right]=-C_{\alpha},\left[H, T_{\alpha}\right]=T_{\alpha}\right)$. The relations $(2.8)-$ (2.9) can be easily obtained in the projective realization of $\bar{M}$ where the transformation $g_{c}$ is represented by a rotation of an angle $\frac{\pi}{2}$ in the plane $\left(i \vec{e}_{0}, \vec{e}_{D}\right)\left(\in \mathbb{C}^{D+2}\right.$, 
see for more detail [26] Appendix A). Note that there is an involutive antilinear automorphism $\star: c_{\mathbb{C}} \rightarrow c_{\mathbb{C}}$ leaving invariant the real algebra $c$, i.e.,

$$
\begin{gathered}
\Omega^{\star \star}=\Omega, \quad\left(\lambda \Omega+\lambda^{\prime} \Omega^{\prime}\right)^{\star}=\bar{\lambda} \Omega^{\star}+\overline{\lambda^{\prime}} \Omega^{\prime \star}, \quad\left[\Omega, \Omega^{\prime}\right]^{\star}=\left[\Omega^{\star}, \Omega^{\prime \star}\right], \\
\Omega_{a b}^{\star}=\Omega_{a b} \quad \Longrightarrow \quad P_{\mu}^{\star}=-P_{\mu}, \quad K_{\mu}^{\star}=-K_{\mu}, \quad H^{\star}=-H, \quad T_{\alpha}^{\star}=C_{\alpha}
\end{gathered}
$$

for $\lambda, \lambda^{\prime} \in \mathbb{C}, \Omega, \Omega^{\prime} \in \mathcal{c}_{\mathbb{C}}, \mu=0, \ldots, D-1$ and $\alpha=1, \ldots, D$. In fact, the real generators $T_{\alpha}, C_{\alpha}, \Omega_{\alpha \beta}, H(\alpha, \beta=1, \ldots, D)$ span an Euclidean real form $(\cong$ $\operatorname{spin}(D+1,1))$ of the complex conformal algebra.

From a group theoretic point of view the compactified Minkowski space $\bar{M}$ is a homogeneous space of the conformal group $\mathcal{C}$ characterized by the stabilizers of the points. For the tip $p_{\infty}$ of the light cone at infinity $K_{\infty}:=\bar{M} \backslash M$ (recall that the isotropy relation extends to a conformally invariant relation on $\bar{M}$ ) the stabilizer is exactly the Weyl group: the Poinaré group with dilations. In more detail, the Lie algebra of the stabilizer of $p_{\infty}$ is spanned by the generators $\left\{i P_{\mu}, \Omega_{\mu \nu}, \Omega_{-1 D}\right\}$, while the Lie algebra of the stabilizer of the origin $p_{0}$ (corresponding to $x=0 \in M$ ) is spanned by $\left\{i K_{\mu}, \Omega_{\mu \nu}, \Omega_{-1 D}\right\}$. Thus every chart in $\bar{M}$ as well as in $\bar{M}_{\mathbb{C}}$ can be uniquely characterized, as a vector space, by a pair $(p, q)$ of mutually nonisotropic points: the origin $p$ of the chart and the tip $q$ of the its light cone complement. For the Minkowski space chart the stabilizer of the pair $(p, q) \equiv\left(p_{0}, p_{\infty}\right)$ is the Cartesian product of the (one-parameter) dilation and the Lorentz subgroups with Lie algebra spanned by $\left\{\Omega_{-1 D}, \Omega_{\mu \nu}\right\}$. The $z$-chart introduced above is characterized by the pair of mutually conjugate points $(p, q)=\left(i e_{0},-i e_{0}\right)\left(e_{0}:=(1, \mathbf{0}) \in M\right.$, so that $\left.p \in \mathfrak{T}_{+} \subset M_{\mathbb{C}}\right)$ and the stabilizer $\mathcal{K}_{\mathbb{C}}$ of this pair is a $\star$-invariant subgroup of $\mathcal{C}_{\mathbb{C}}$. The real part of $\mathcal{K}_{\mathbb{C}}$ coincides with the maximal compact subgroup $\mathcal{K}$ which is generated by two mutually commuting subgroups: the $U(1)$-group $\left\{e^{2 \pi i t H}\right\}$ and the $\operatorname{Spin}(D)$ group acting on $z$ via real (Euclidean) rotations $\left(\mathcal{K} \cong U(1) \times \operatorname{Spin}(D) \mathbb{Z}_{2}\right)$. Since the points $p$ and $q$ are mutually conjugate, $\mathcal{K}$ is also the real part of the stabilizer of the origin in the $z$-chart. In fact, $T_{+}(2.5)$ is isomorphic to the homogeneous space $\mathcal{C} / \mathcal{K}$ of $\mathcal{C}$ (cf. [36]). Note that the complex Lie algebra of the stabilizer of $z=0$ is spanned by the generators $\left\{C_{\alpha}, H, \Omega_{\alpha \beta}\right\}$.

Remark 2.1. In the familiar realization of $\bar{M}$ as the Dirac projective quadric [9],

$$
\bar{M}=Q / \mathbb{R}^{*}, \quad Q=\left\{\vec{\xi} \in \mathbb{R}^{D, 2} \backslash\{0\}: \vec{\xi}^{2}:=\boldsymbol{\xi}^{2}+\xi_{D}^{2}-\xi_{0}^{2}-\xi_{-1}^{2}\left(=\xi^{a} \eta_{a b} \xi^{b}\right)=0\right\} .
$$

the Minkowski space coordinates $x$ and the complex coordinates $z$ of (1.3) are expressed by

$$
x^{\mu}=\frac{\xi^{\mu}}{\xi^{D}+\xi^{-1}}, \quad z^{\alpha}=\frac{\xi^{\alpha}}{\xi^{-1}-i \xi^{0}} .
$$

Remark 2.2. Only Lorentz types of signatures $(D-1,1)$ or $(1, D-1)$ possess the remarkable property that there exist affine charts covering the corresponding conformally compactified real space ([26] Proposition A.1). Moreover, every such chart is characterized by the condition that the tip $q$ of the light cone complement 
belongs to the union $T_{+} \cup T_{-}\left(T_{-}:=\left(T_{+}\right)^{*}\right.$ is the image in the $z$-coordinates of the backward tube $\mathfrak{T}_{-}$). If $q \in T_{ \pm}$then $T_{\mp}$ is also covered by the chart.

\subsection{Wightman axioms for conformal field theory in the analytic picture}

We proceed with a brief survey of the axiomatic QFT with GCI. First, one assumes the existence of a vector bundle over the complex compactified Minkowski space $\bar{M}_{\mathbb{C}}$ called the field bundle. It is endowed with an action of the conformal group $\mathcal{C}_{\mathbb{C}}$ via (bundle) automorphisms. Thus for every point $p \in \bar{M}_{\mathbb{C}}$ its stabilizer $\mathcal{C}_{p}$ will act by a representation $\pi_{p}$ on the (finite dimensional) fibre $F_{p}$ over $p$. Then if $p$ is the origin of some affine chart in $\bar{M}_{\mathbb{C}}$, e.g., the $z$-chart, we can trivialize the bundle over the chart using the coordinate translations $t_{w}(z)\left(\equiv e^{w \cdot T}(z)\right)=z+w$ so that the action of $\mathcal{C}_{\mathbb{C}}$ in this trivialization will take the form

$$
\left(z=\left\{z^{\alpha}\right\}, \phi=\left\{\phi_{A}\right\}\right) \underset{g}{\longmapsto}\left(g(z), \pi_{z}(g) \phi=\left\{\pi_{z}(g)_{A}^{B} \phi_{B}\right\}\right) \in \mathbb{C}^{D} \times F
$$

where $\left\{\phi_{A}\right\}$ are some (spin-tensor) coordinates in the fibre $F:=F_{0}$ over the origin $z=0$ and the matrix valued function $\pi_{z}(g)=\left\{\pi_{z}(g)_{A}^{B}\right\}\left(g \in \mathcal{C}_{\mathbb{C}}\right)$, regular in the domain of $g$ and called cocyle, is characterized by the properties

$$
\pi_{z}\left(g_{1} g_{2}\right)=\pi_{g_{2}(z)}\left(g_{1}\right) \pi_{z}\left(g_{2}\right), \quad \pi_{z}\left(t_{w}\right)=\mathbb{I}_{F}\left(\Leftrightarrow \pi_{z}(g)=\pi_{0}\left(t_{g(z)}^{-1} g t_{z}\right)\right) .
$$

The fibre $F$ is the space of (classical) field values and the coordinates $\phi_{A}$ correspond to the collection of local fields in the theory. An example of a field bundle is the electro-magnetic field defined as the bundle of 2-forms $F_{\alpha \beta} d z^{\alpha} \wedge d z^{\beta}=F_{\mu \nu}^{M} d x^{\mu} \wedge d x^{\nu}$ over $\bar{M}$.

The axiomatic assumptions of the GCI QFT are the Wightman axioms [34] and the condition of GCI for the correlation functions [29]. As proven in [29], Theorem 3.1, GCI is equivalent to the rationality of the (analytically continued) Wightman functions. Thus the vacuum $n$-point correlation functions in the theory can be considered as meromorphic sections of the $n$th tensor power (over $\bar{M}_{\mathbb{C}}^{\times n}$ ) of the field bundle and hence, for every affine chart in $\bar{M}_{\mathbb{C}}$ we obtain a system of rational correlation functions over the chart. This provides the general scheme for the passage from the GCI QFT over Minkowski space to the theory over a complex affine chart which contains the forward "tube" $T_{+}(2.5)$ - see [26] Sect. 9.

The (analytic) $z$-picture of a GCI QFT is equivalent to the theory of vertex algebras ([2] [19] [3] [11]) in higher dimensions (see [26]). We proceed to formulate the analogue of Wightman axioms [34] in this picture.

The quantum fields $\phi_{A}(z)(A=1, \ldots, I$ for $I=\operatorname{dim} F)$ will be treated as formal power series in $z$ and $\frac{1}{z^{2}}$. This is possible because of the analytic properties of the fields in a GCI Wightman QFT ([26] Theorem 9.1). Using harmonic polynomials one can uniquely separate the integer powers of the interval $z^{2}$ due to the following (known) fact: for every polynomial $p(z)$ there exist unique polynomials $h(z)$ and 
$q(z)$ such that $h(z)$ is harmonic and $p(z)=h(z)+z^{2} q(z)$. Thus, if we fix a basis $\left\{h_{\sigma}^{(m)}(z)\right\}$ of homogeneous harmonic polynomials of degree $m$, for every $m=$ $0,1, \ldots$, we can write our fields $\phi_{A}(z)$ in a unique way as formal series in the monomials $\left(z^{2}\right)^{n} h_{\sigma}^{(m)}(z)$ for $n \in \mathbb{Z}, m=0,1, \ldots$ and the index $\sigma$ takes values in a finite set $I_{m}$. In such a way we end up with the following axiom:

Fields $(F)$. The fields are represented by (nonzero) formal series

$$
\phi_{A}(z)=\sum_{n \in \mathbb{Z}} \sum_{m=0}^{\infty} \sum_{\sigma} \phi_{A\{n, m, \sigma\}}\left(z^{2}\right)^{n} h_{\sigma}^{(m)}(z),
$$

with coefficients $\phi_{A\{n, m, \sigma\}}$ which are operators acting on a common invariant dense domain $\mathcal{D}$ of the Hilbert space $\mathcal{H}$ of physical states. We require that for every vector state $\Psi \in \mathcal{D}$ there exists a constant $N_{\Psi} \in \mathbb{N}$ such that $\phi_{A\{n, m, \sigma\}} \Psi=0$ for all $n \leqslant-N_{\Psi}, m=0,1, \ldots$ and all possible values of $\sigma$, or equivalently, $\left(z^{2}\right)^{N_{\Psi}} \phi_{A}(z) \Psi$ is a formal power series with no negative powers. (This requirement is related to the energy positivity, stated below in the axiom $(S C)$.)

As the properties of the field $\phi_{A}(z)$ do not dependent of the choice on $\left\{h_{\sigma}^{(m)}(z)\right\}$ we may also write it in a basis independent form:

$$
\phi_{A}(z)=\sum_{n \in \mathbb{Z}} \sum_{m=0}^{\infty} \phi_{A\{n, m\}}(z)\left(z^{2}\right)^{n}
$$

where $\phi_{A\{n, m\}}(z)\left(=\sum_{\sigma} \phi_{A\{n, m, \sigma\}} h_{\sigma}^{(m)}(z)\right)$ are operator valued homogeneous harmonic polynomials. We shall use this more concise presentation in studding examples of free fields (Sect. 4). Using an (arbitrary) basis $\left\{h_{\sigma}^{(m)}(z)\right\}$, on the other hand, makes more transparent the algebraic manipulations of formal power series in this and next sections.

The next axiom introduces the conformal symmetry of the theory.

Covariance $(C)$. There exists a unitary representation, $U(g)$ of the real conformal group $\mathcal{C}$ on the Hilbert space $\mathcal{H}$ such that the hermitian generators of the conformal Lie algebra $c$ leave invariant the fields' domain $\mathcal{D}$. We also require the existence of a rational matrix-valued function $\left\{\pi_{z}(g)_{A}^{B}\right\}_{A, B=1, \ldots, I}$ depending on $z \in \mathbb{C}^{D}$ and $g \in \mathcal{C}$, regular for $z$ in the domain of $g$ on $\mathbb{C}^{D}$, and such that it satisfies the properties (2.12). Then the fields $\phi_{A}(z)$ are assumed to satisfy infinitesimal conformal covariance, formally written as:

$$
\begin{aligned}
& \left.\frac{d}{d t}\left(U\left(e^{t \Omega}\right) \phi_{A}(z) U\left(e^{t \Omega}\right)^{-1}\right)\right|_{t=0}= \\
& =\left.\frac{d}{d t}\left(\left[\pi_{z}\left(e^{t \Omega}\right)^{-1}\right]_{A}^{B} \phi_{B}\left(e^{t \Omega}(z)\right)\right)\right|_{t=0}
\end{aligned}
$$

for $\Omega \in \operatorname{spin}(D, 2)$. 
It is simpler to write down the field covariance law if we further assume that our fields are transforming under an elementary induced representation of the conformal group $\mathcal{C}$. This means that the cocycle $\pi_{z}(g)$ is trivial at $z=0$ for $g=e^{a \cdot C}$ and it is thus determined by a representation of the maximal compact subgroup $\mathcal{K}$ of $\mathcal{C}$ :

$$
\pi_{z}\left(e^{i t H}\right)_{A}^{B}=e^{i t d_{A}} \delta_{A}^{B}, \quad \pi_{z}\left(e^{t \Omega_{\alpha \beta}}\right)_{A}^{B}=\left[e^{t \pi_{0}\left(\Omega_{\alpha \beta}\right)}\right]_{A}^{B},
$$

where $d_{A}$ are positive numbers called dimensions of the corresponding fields $\phi_{A}$. Under this additional assumption we can present Eq. (2.15) in a more concrete form:

$$
\begin{aligned}
{\left[T_{\alpha}, \phi_{A}(z)\right]=} & \partial_{z^{\alpha}} \phi_{A}(z) \\
{\left[H, \phi_{A}(z)\right]=} & z \cdot \partial_{z} \phi_{A}(z)+d_{A} \phi_{A}(z) \\
{\left[\Omega_{\alpha \beta}, \phi_{A}(z)\right]=} & \left(z^{\alpha} \partial_{z^{\beta}}-z^{\beta} \partial_{z^{\alpha}}\right) \phi_{A}(z)+\pi_{0}\left(\Omega_{\alpha \beta}\right)_{A}^{B} \phi_{B}(z) \\
{\left[C_{\alpha}, \phi_{A}(z)\right]=} & \left(z^{2} \partial_{z^{\alpha}}-2 z^{\alpha} z \cdot \partial_{z}\right) \phi_{A}(z)-2 z^{\alpha} d_{A} \phi_{A}(z)+ \\
& +2 z^{\beta} \pi_{0}\left(\Omega_{\beta \alpha}\right)_{A}^{B} \phi_{B}(z)
\end{aligned}
$$

where $\partial_{z^{\alpha}}$ stands for the partial (formal) derivative $\frac{\partial}{\partial z^{\alpha}}$.

We further assume that the hermitian conjugate $\phi_{A}(z)^{*}$ of each $\phi_{A}(z)$ belongs to the linear span of the set $\left\{\phi_{A}\right\}$.

Field conjugation law $\left({ }^{*}\right)$. For every $\Psi_{1}, \Psi_{2} \in \mathcal{D}$ and for any field $\phi_{A}$ there exists a conjugate field $\stackrel{*}{\phi}_{A}$ such that:

$$
\left\langle\Psi_{1} \mid \phi_{A}(z) \Psi_{2}\right\rangle=\left\langle\pi_{R_{D}(\bar{z})}\left(j_{W}^{-1}\right)_{A}^{B}{\stackrel{*}{\phi_{B}}}\left(z^{*}\right) \Psi_{1} \mid \Psi_{2}\right\rangle,
$$

where $j_{W}$ is defined by (2.6) and (2.7). The exact meaning of Eq. (2.21) is provided by the fact that both sides are finite series, i. e. polynomials in $z$ and $1 / z^{2}$ (see [26] Remark 8.1). The correspondence $\phi_{A} \mapsto \stackrel{*}{\phi}_{A}$ gives rise to an antilinear involution in the standard fibre $F$ of the field bundle, invariant under the action of $\mathcal{C}$.

The next axiom states energy positivity and determines the physical vacuum.

Spectral condition $(S C)$. The conformal time generator $H$ is represented on $\mathcal{H}$ by a positive operator. There is only one norm 1 conformally invariant vector $|0\rangle \in \mathcal{H}$ (up to phase factor) and it is contained in the fields' domain $\mathcal{D}$.

We shall now formulate a strong form of the locality axiom also called Huygens' principle stating that the fields are independent for nonisotropic separations.

Strong Locality or Huygens' principle $(S L)$. Every field $\phi_{A}(z)$ is assumed to have a fixed statistical parity $p_{A}=0,1$ and there exist positive integers $M_{A B}$ such that:

$$
\left(z_{12}^{2}\right)^{M_{A B}}\left(\phi_{A}\left(z_{1}\right) \phi_{B}\left(z_{2}\right)-(-1)^{p_{A} p_{B}} \phi_{B}\left(z_{2}\right) \phi_{A}\left(z_{1}\right)\right)=0
$$

where $z_{12}:=z_{1}-z_{2}$. 
Remark 2.3. When we deal with formal power series it is more convenient to use weaker (infinitesimal) conformal invariance but a stronger locality axiom. Indeed, for rational functions, GCI follows from infinitesimal conformal invariance. Thus within the Wightman framework, the two pairs of axioms: (1) ordinary locality and GCI, (2) strong locality and infinitesimal conformal invariance, are completely equivalent.

Completeness. The set of vectors $|0\rangle, \phi_{A_{1}\left\{n_{1}, m_{1}, \sigma_{1}\right\}} \ldots \phi_{A_{k}\left\{n_{k}, m_{k}, \sigma_{k}\right\}}|0\rangle$, for all $k \in N$ and all possible values of the indices of the $\phi$ 's, spans the fields' domain $\mathcal{D}$.

This completes our analogue of Wightman axioms in the $z$-picture. Theorems 9.2 and 9.3 of [26] allow one to state the following general result:

Theorem 2.1. There is a one-to-one correspondence between the finite systems of Wightman fields with GCI correlation functions [29] and the systems of formal series satisfying the above axioms.

Using the fact that the cocycle $\pi_{z}(g)$ is meromorphic (even rational) in $g$ and $z$ we can continue it to $\mathcal{C}_{\mathbb{C}}$ and write down the explicit connection between the Wightman fields $\phi_{A}^{M}(x)$ on the Minkowski space and the analytic picture fields $\phi_{A}(z)$ :

$$
\phi_{A}^{M}(x)=\pi_{z}\left(g_{c}^{-1}\right)_{A}^{B} \phi_{B}\left(g_{c}(x)\right) \quad\left(z=g_{c}(x)\right)
$$

where $g_{c}$ is the transformation $(2.3)$ viewed as an element of $\mathcal{C}_{\mathbb{C}}$. Eq. (2.23) is the precise expression of the fact that the fields $\phi_{A}^{M}(x)$ and $\phi_{A}(z)$ are different coordinate expressions of the same (generalized, operator-valued) section of the field bundle over $\bar{M}$. For example, for the electromagnetic field Eq. (2.23) is equivalent to:

$$
F_{\mu \nu}^{M}(x) d x^{\mu} \wedge d x^{\nu}=F_{\alpha \beta}(z) d z^{\alpha} \wedge d z^{\beta} .
$$

The rigorous meaning of Eqs. (2.23) and (2.24) includes, on one hand, the extension of the operator valued functions $\phi_{A}^{M}(x)$ to a larger class of test functions which correspond to coordinate expressions of arbitrary smooth sections over $\bar{M}$. This can be done using the GCI condition (see [29] Proposition 2.1). On the other hand, using the positivity of the scalar product of the Hilbert state space one can easily extend our formal field series (2.15) to generalized operator valued functions over $\bar{M}$ (in the parametrization (1.3)).

We now proceed to introduce the real compact picture representation which is more convenient in studying finite temperature correlation functions.

For a local field $\phi(z)$ of dimension $d$ we set $\phi(\zeta, u)$ to be a formal Fourier series in $e^{2 \pi i \zeta} \in \mathbb{S}^{1}$ and $u \in \mathbb{S}^{D-1}$ defined as:

$$
\begin{aligned}
\phi(\zeta, u) & =e^{2 \pi i d \zeta} \phi\left(e^{2 \pi i \zeta} u\right)=\sum_{\nu \in d+\mathbb{Z}} \sum_{m=0}^{\infty} \phi_{-\nu m}(u) e^{2 \pi i \nu \zeta}, \\
\phi_{-\nu m}(u) & =\sum_{\sigma} \phi_{-\nu, m, \sigma} h_{\sigma}^{(m)}(u) .
\end{aligned}
$$


(The space of harmonic polynomials, $\operatorname{Span}_{\mathbb{C}}\left\{h_{\sigma}^{(m)}(u)\right\}_{m, \sigma}$, is identified with the algebra of complex polynomials restricted to the sphere $\mathbb{S}^{D-1}$.) Then the connection with the previous analytic picture modes is:

$$
\phi_{\{n, m, \sigma\}}=\phi_{\nu, m, \sigma} \quad \text { for } \quad \nu=-d-2 n-m \quad\left(n=-\frac{\nu+m+d}{2}\right) .
$$

Note that the index $n$ in the analytic picture modes $\phi_{\{n, m, \sigma\}}$ is always integer while in the compact picture modes, $\phi_{\nu, m, \sigma}$, it is integer or half-integer depending on $d$ (which is reflected in the first sum in (2.25)). In accord with the commutation relation (2.18) we obtain

$$
\begin{gathered}
{\left[H, \phi_{\nu, m}(u)\right]=-\nu \phi_{\nu, m}(u), \quad e^{2 \pi i t H} \phi_{\nu, m}(u) e^{-2 \pi i t H}=e^{-2 \pi i t \nu} \phi_{\nu, m}(u)} \\
{\left[H, \phi_{\{n, m\}}(z)\right]=(d+2 n+m) \phi_{\{n, m\}}(z)}
\end{gathered}
$$

It follows that $2 \pi i H$ acts as a translation generator in $\zeta$ in accord with Eq. (1.6).

As a realization of the above axioms we will consider the case of a neutral scalar field $\phi(z) \equiv \phi^{(d)}(z)$ of dimension $d$. Its 2-point function is proportional to the unique scalar conformal invariant function of dimension $d$,

$$
\left\langle 0\left|\phi\left(z_{1}\right) \phi\left(z_{2}\right)\right| 0\right\rangle=\frac{1}{\left(z_{12}^{2}\right)^{d}} \quad\left(z_{12}=z_{1}-z_{2}\right)
$$

viewed as a Taylor series in the second argument, $z_{2}$, with coefficients - rational functions in $z_{1}$ (see Theorem 3.3 below). The field cocycle is $\pi_{z}(g)=\omega(g, z)^{-d}$, where $\omega(g, z)$ is a quadratic polynomial in $z\left(\omega(g, z)=\left(\operatorname{det}\left(\frac{\partial g(z)^{\alpha}}{\partial z^{\beta}}\right)\right)^{-\frac{1}{D}}\right.$, see [29] Eq. (A.5)). (Note that the Minkowski space transform of the correlation function (2.28) is proportional to $\frac{1}{x_{12}^{2}+i 0 x_{12}^{0}}-$ cf. with Eq. (5.17).) The hermiticity of the field is expressed by

$$
\phi(z)^{*}=\frac{1}{\left(\bar{z}^{2}\right)^{d}} \phi\left(z^{*}\right) \quad\left(z^{*}=\frac{\bar{z}}{\bar{z}^{2}}, \phi(z) \equiv \stackrel{*}{\phi}(z)\right)
$$

since $\omega\left(j_{W}, z\right)=z^{2}$. This conjugation law simplifies in the compact picture, since we are using real coordinates $(\zeta, u)$; the hermiticity condition for the field modes reads:

$$
\phi_{\nu, m, \sigma}^{*}=\phi_{-\nu, m, \sigma} .
$$

\section{GCI Correlation Functions as Meromorphic Functions}

\subsection{Rationality of the vacuum correlation functions}

Theorem 2.1 implies the rationality of the Wightman functions as well as the analyticity properties of the fields. Since these facts are of major importance we shall prove them independently. We begin with stating some basic properties of the formal series which arise in the analytic picture of GCI QFT. 
We introduce, following [26], the space $V \llbracket z, 1 / z^{2} \rrbracket$ of formal series

$$
v(z)=\sum_{n \in \mathbb{Z}} \sum_{m=0}^{\infty} \sum_{\sigma} v_{\{n, m, \sigma\}}\left(z^{2}\right)^{n} h_{\sigma}^{(m)}(z)
$$

with coefficients $v_{\{n, m, \sigma\}}$ belonging to a complex vector space $V$. The space of finite series of $V \llbracket z, 1 / z^{2 \rrbracket}$ will be denoted by $V\left[z, 1 / z^{2}\right]$. Obviously, $\mathbb{C}\left[z, 1 / z^{2}\right]$ is a complex algebra and $V\left[z, 1 / z^{2}\right]$ is a module over this algebra. Nevertheless, the product between a series $f(z) \in \mathbb{C} \llbracket z, 1 / z^{2} \rrbracket$ and a series $v(z) \in V \llbracket z, 1 / z^{2} \rrbracket$ is not defined in general but it is not difficult to define the product $f(z) v(z)$ if $f(z) \in \mathbb{C}\left[z, 1 / z^{2}\right]$ (thus turning $V \llbracket z, 1 / z^{2} \rrbracket$ into a $\mathbb{C}\left[z, 1 / z^{2}\right]$-module). We emphasize that all the products between formal series throughout this paper will be treated in purely algebraic sense, i.e., every coefficient of the product series should be obtained by a finite number operations of summation and multiplication on the coefficients of the initial series.

On the other hand, the space of Taylor series $V \llbracket z \rrbracket$ in $z$, with coefficients in $V$, is naturally identified with the subspace of $V \llbracket z, 1 / z^{2} \rrbracket$ of formal series (3.1) whose sum in $n$ runs from 0 to $\infty$. Evidently, $V \llbracket z \rrbracket$ is a module over the algebra $\mathbb{C} \llbracket z \rrbracket$ (i.e., the product $f(z) v(z)$ is well defined for $f(z) \in \mathbb{C} \llbracket z \rrbracket$ and $v(z) \in V \llbracket z \rrbracket)$. Similarly, $V[z]$ (the space of polynomials in $z$ with coefficients in $V$ ) is a subspace of $V\left[z, 1 / z^{2}\right]$ and it is a module over the polynomial algebra $\mathbb{C}[z]$. There is a larger space of formal series of $\mathbb{C} \llbracket z, 1 / z^{2} \rrbracket$ than $\mathbb{C} \llbracket z \rrbracket$ which still possesses a complex algebra structure: this is the space $\mathbb{C} \llbracket z \rrbracket_{z^{2}}$ of those series $f(z) \in \mathbb{C} \llbracket z, 1 / z^{2} \rrbracket$ whose sum in $n$ in (3.1) is bounded below. The more general spaces $V \llbracket z \rrbracket_{z^{2}}$ are defined in a similar way and we have in fact a shorter equivalent definition

$$
v(z) \in V \llbracket z \rrbracket_{z^{2}} \quad \Leftrightarrow \quad\left(z^{2}\right)^{N} v(z) \in V \llbracket z \rrbracket \text { for } N \gg 0 .
$$

Remark 3.1. The notation $\mathbb{C} \llbracket z \rrbracket_{z^{2}}$ comes from commutative algebra: for a commutative ring $R$ and $f \in R$ the localized ring $R_{f}$ is defined as the ring of "ratios" $\frac{a}{f^{n}}$ for $a \in R$ and $n=0,1, \ldots$ (more precisely, this is the quotient ring $R[t] /(f t-1$ ) of the ring $R[t]$ of polynomials in the one-dimensional variable $t$ over ideal generated by $f t-1-$ see [1]). In a similar way, if $V$ is a module over the ring $R$ then the localized space $V_{f}$ is defined as a module over the localized ring $R_{f}$. Note that $\mathbb{C}\left[z, 1 / z^{2}\right] \equiv \mathbb{C}[z]_{z^{2}}$.

Proposition 3.1. Let $V$ be a complex vector space.

(a) The space $\mathbb{C} \llbracket z \rrbracket_{z^{2}}$ is a complex algebra containing $\mathbb{C} \llbracket z \rrbracket$ as a subalgebra and $V \llbracket z \rrbracket_{z^{2}}$ is a module over this algebra that extends the module structure of $V \llbracket z \rrbracket$ over the algebra $\mathbb{C} \llbracket z \rrbracket$.

(b) There are no zero divisors in the $\mathbb{C} \llbracket z \rrbracket_{z^{2}}$ module $V \llbracket z \rrbracket_{z^{2}}$, i.e., if $f(z) \in \mathbb{C} \llbracket z \rrbracket_{z^{2}}$ and $v(z) \in V \llbracket z \rrbracket_{z^{2}}$ are such that $f(z) v(z)=0$ then either $f=0$ or $v=0$.

(c) If $w$ is another $D$-dimensional formal variable and

$$
V \llbracket z \rrbracket_{z^{2}} \llbracket w \rrbracket_{w^{2}}:=\left(V \llbracket z \rrbracket_{z^{2}}\right) \llbracket w \rrbracket_{w^{2}}
$$


then the polynomial $(z-w)^{2}$ is invertible in $V \llbracket z \rrbracket_{z^{2}} \llbracket w \rrbracket_{w^{2}}$ and its inverse, denoted by $\iota_{z, w} \frac{1}{(z-w)^{2}}$, is the Taylor series of $\frac{1}{(z-w)^{2}}$ in $w$ with coefficients belonging to $\mathbb{C}\left[z, 1 / z^{2}\right]$ (i.e., $\iota_{z, w} \frac{1}{(z-w)^{2}}:=\sum_{n=0}^{\infty} \frac{(-1)^{n}}{n !} \partial_{z^{\alpha_{1}}} \ldots \partial_{z^{\alpha_{n}}}\left(\frac{1}{z^{2}}\right) w^{\alpha_{1}}$ $\left.\ldots w^{\alpha_{n}}\right)$.

The proof of Proposition 3.1 is quite simple. We just remark that the product of $f(z) \in \mathbb{C} \llbracket z \rrbracket_{z^{2}}$ and $v(z) \in V \llbracket z \rrbracket_{z^{2}}$ can be defined as $\left(z^{2}\right)^{-N_{1}-N_{2}}\left(\left(z^{2}\right)^{N_{1}} f(z)\right)$ $\left(\left(z^{2}\right)^{N_{2}} v(z)\right)$ for $N_{1}, N_{2} \gg 0$, according to Eq. (3.2), and does not depend on $N_{1}$ and $N_{2}$. Then condition (b) follows from the absence of zero divisors in the $\mathbb{C} \llbracket z \rrbracket$-module $V \llbracket z \rrbracket$.

Having several $D$-dimensional variables $z_{1}, \ldots, z_{n}$ one can inductively define

$$
V \llbracket z_{1},{ }^{1} z_{1}^{2} ; \ldots ; z_{n}, 1 / z_{n}^{2} \rrbracket:=\left(V \llbracket z_{1}, 1 / z_{1}^{2} ; \ldots ; z_{n-1},{ }^{1} / z_{n-1}^{2} \rrbracket\right) \llbracket z_{n},{ }^{1} z_{n}^{2} \rrbracket .
$$

A different order of $z_{1}, \ldots z_{n}$ in the right hand side of (3.3) will correspond to another way of summations in the formal series. Nevertheless, the order of $z_{k}$ in the successive localizations $V \llbracket z_{1} \rrbracket_{z_{1}^{2}} \ldots \llbracket z_{n} \rrbracket_{z_{n}^{2}}$ is important. For example, $\iota_{z, w} \frac{1}{(z-w)^{2}}$ and $\iota_{w, z} \frac{1}{(z-w)^{2}}$ are different formal series of $\mathbb{C} \llbracket z,{ }^{1} / z^{2} ; w,{ }^{1} / w^{2} \rrbracket$. From Proposition $3.1(c)$ it follows that

$$
(z-w)^{2}\left(\iota_{z, w} \frac{1}{(z-w)^{2}}-\iota_{w, z} \frac{1}{(z-w)^{2}}\right)=0,
$$

so we see that in the $\mathbb{C}\left[z,{ }^{1} / z^{2} ; w,{ }^{1} / w^{2}\right]-$ module $\mathbb{C} \llbracket z,{ }^{1} / z^{2} ; w,{ }^{1} / w^{2} \rrbracket$ there are zero divisors (the same is true in any $\mathbb{C}\left[z, 1 / z^{2}\right]$-module $V \llbracket z,{ }^{1} / z^{2} \rrbracket$ ).

The spaces of successive localizations play important role in the analytic picture GCI QFT since, according to Axiom $(F)$, we have

$$
\phi_{A_{1}}\left(z_{1}\right) \ldots \phi_{A_{n}}\left(z_{n}\right) \Psi \in \mathcal{D} \llbracket z_{1} \rrbracket_{z_{1}^{2}} \ldots \llbracket z_{n} \rrbracket_{z_{n}^{2}}
$$

for any state vector $\Psi$ belonging to the fields' domain $\mathcal{D}$. They are convenient, on one hand, since these spaces have no zero divisors and, on the other, since products of the type $\prod_{1 \leqslant k<l \leqslant n} z_{k l}^{2}$ are invertible in the algebra $\mathbb{C} \llbracket z_{1} \rrbracket_{z_{1}^{2}} \ldots \llbracket z_{n} \rrbracket_{z_{n}^{2}}$.

We will further assume in this section the validity of the postulates of Sect. 2.2.

\section{Proposition 3.2.}

(a) The formal series $\phi_{A}(z)|0\rangle$ is nonzero and does not contain negative powers of $z^{2}$, i.e. $\phi_{A}(z)|0\rangle \in \mathcal{D} \llbracket z \rrbracket$, for $A=1, \ldots, I$.

(b) The field dimensions $d_{A}$ are positive integers or half integers. They are related to the statistical parities $p_{A}(=0,1)$ by the equality $(-1)^{2 d_{A}}=(-1)^{p_{A}}$. 
(c) The operator $H$ has a pure discrete spectrum containing only integer or half integer nonnegative numbers. The fields' domain $\mathcal{D}$ coincides with the linear span of all eigenvectors of $H, i$. e. this is the finite energy space. Moreover, the domain $\mathcal{D}$ is invariant under the maximal compact subgroup $\mathcal{K}$ of the conformal group and decomposes under its action into a direct sum of finite dimensional irreducible representations.

Proof. (a) Take the formal series $F(z, w)=e^{w \cdot T} \phi_{A}(z)|0\rangle$. The commutation relation (2.17) implies that $\partial_{z^{\alpha}} F(z, w)=\partial_{w^{\alpha}} F(z, w)$. Suppose, on the other hand, in accord with the existence of harmonic decomposition and Axiom $(F)$ that $F(z, w)=\left(z^{2}\right)^{-N} h(z, w)+\left(z^{2}\right)^{-N+1} g(z, w)$, where $h(z, w)$ and $g(z, w)$ are formal series with no negative powers of $z$ and $w$ and $h$ is in addition nonzero and harmonic with respect to $z$. If we now assume that $N>0$ it will turn out that $\partial_{z^{\alpha}} F(z, w)=\left(z^{2}\right)^{-N-1} h_{1}(z, w)+\left(z^{2}\right)^{-N} g_{1}(z, w)$ where $h_{1}(z, w)$ and $g_{1}(z, w)$ are series with similar properties. Then the equation $\partial_{z^{\alpha}} F(z, w)=\partial_{w^{\alpha}} F(z, w)$ will imply an equality of type $h_{2}(z, w)=z^{2} g_{2}(z, w)$ for the series $h_{2}(z, w)$ and $g_{2}(z, w)$ with no negative powers, $h_{2}$ being nonzero and harmonic with respect to $z$. But this would contradict the uniqueness of the harmonic decomposition.

The series $\phi_{A}(z)|0\rangle$ is nonzero: otherwise Axioms $(F)$ and $(S L)$ imply that $\left(\prod_{k<l} z_{k l}^{2}\right)^{N} \phi_{A}\left(z_{1}\right) \phi_{A_{2}}\left(z_{2}\right) \ldots \phi_{A_{n}}\left(z_{n}\right)|0\rangle=0$, for large $N \in \mathbb{N}$, and we can cancel the polynomial prefactor due to the fact that in the $\mathbb{C} \llbracket z_{1} \rrbracket_{z_{1}^{2}} \ldots \llbracket z_{n} \rrbracket_{z_{n}^{2}}$-module $\mathcal{D} \llbracket z_{1} \rrbracket_{z_{1}^{2}} \ldots \llbracket z_{n} \rrbracket_{z_{n}^{2}}$ there are no zero divisors; then the completeness axiom would imply that the field $\phi_{A}(z)$ is zero.

(b) The positivity of $d_{A}$ is a consequence, on the one hand, of Eq. (2.27) implying $H \phi_{A\{0,0\}}|0\rangle=d_{A} \phi_{A\{0,0\}}|0\rangle\left(\phi_{A\{0,0\}}=\phi_{A\{0,0\}}(z)\right.$ is the mode multiplying the unique, up to proportionality, harmonic polynomial $h_{1}^{(0)}(z)=1$ of degree 0 ) and on the other, of the positivity of $H$ (axiom (SC)). Note also that, according to condition $(a)$, the vector $\left.\phi_{A\{0,0\}}|0\rangle \equiv \phi_{A}(z)|0\rangle\right|_{z=0}$ is nonzero and noncollinear with $|0\rangle$ since otherwise, it will follow that $\phi_{A\{n, m, \sigma\}}|0\rangle=0$ for $n>0$ (and hence, $\phi_{A}(z)|0\rangle=0$ ), or $\phi_{A}(z) \sim \mathbb{I}$, respectively (because $\phi_{A}(z)|0\rangle=e^{z \cdot T} \phi_{A\{0,0\}}|0\rangle$ ). The second statement follows from Proposition 7.1 of [26] and the assumed rationality of the field cocycle (see the covariance axiom).

(c) The set of vectors in the axiom of completeness is actually a set of eigenvectors of $H$ with integer or half-integer eigenvalues. From the commutation relation (2.19) it also follows that every vector of this system is contained in a finite dimensional subrepresentation of the Lie algebra of the maximal compact subgroup.

Remark 3.2. The vector $\Phi_{A}=\left.\phi_{A}(z)|0\rangle\right|_{z=0}$ uniquely characterizes the field $\phi_{A}(z)$ and we have $\phi_{A}(z)|0\rangle=e^{z \cdot T} \Phi_{A}$. Moreover, for every $v \in \mathcal{D}$ there exists a unique translation covariant local field $Y(v, z)$ such that $Y(v, z)|0\rangle=e^{z \cdot T} v$ (see [N03], Sect. 3). This is a compact formulation of the state-field correspondence in 
the vertex algebra approach.

Theorem 3.3. Every scalar product $\left\langle\Psi_{1} \mid \phi_{A_{1}}\left(z_{1}\right) \ldots \phi_{A_{n}}\left(z_{n}\right) \Psi_{2}\right\rangle$ (for arbitrary $\left.\Psi_{1}, \Psi_{2} \in \mathcal{D}\right)$, regarded as a power series, is absolutely convergent in the domain

$$
\begin{aligned}
\mathbb{U}_{n}^{<}:=\{ & \left(z_{1}, \ldots, z_{n}\right) \in \mathbb{C}^{D n}: z_{k}=e^{2 \pi i \zeta_{k}} u_{k}, \zeta_{k} \in \mathbb{C} \text { and } u_{k} \in \mathbb{S}^{D-1} \subset \mathbb{R}^{D} \\
& \left.\left(u_{k}^{2}=1\right) \text { for } k=1, \ldots, n ; \text { Im } \zeta_{k l}<0 \text { for } 1 \leqslant k<l \leqslant n\right\}
\end{aligned}
$$

and its limit is a rational function of the form $\left(\prod_{k=1}^{n} z_{k}^{2}\right)^{-N}\left(\prod_{1 \leqslant k<l \leqslant n} z_{k l}^{2}\right)^{-N}$ $P\left(z_{1}, \ldots, z_{n}\right)$, where $P\left(z_{1}, \ldots, z_{n}\right)$ is a polynomial with coefficients depending on a finite number of modes' scalar products $\left\langle\Psi_{1} \mid \phi_{A_{1}\left\{k_{1}, m_{1}, \sigma_{1}\right\}} \ldots \phi_{A_{n}\left\{k_{n}, m_{1}, \sigma_{1}\right\}} \Psi_{2}\right\rangle$. The Wightman functions $\left\langle 0\left|\phi_{A_{1}}\left(z_{1}\right) \ldots \phi_{A_{n}}\left(z_{n}\right)\right| 0\right\rangle$ (viewed as rational functions) are in addition globally conformal invariant:

$$
\begin{gathered}
\left\langle 0\left|\phi_{A_{1}}\left(z_{1}\right) \ldots \phi_{A_{n}}\left(z_{n}\right)\right| 0\right\rangle= \\
=\left\langle 0\left|\left[\pi_{z}(g)^{-1}\right]_{A_{1}}^{B_{1}} \ldots\left[\pi_{z}(g)^{-1}\right]_{A_{n}}^{B_{n}} \phi_{B_{1}}\left(g\left(z_{1}\right)\right) \ldots \phi_{B_{n}}\left(g\left(z_{n}\right)\right)\right| 0\right\rangle,
\end{gathered}
$$

and $\mathbb{Z}_{2}$-symmetric in the sense

$$
\left\langle 0\left|\phi_{A_{\sigma(1)}}\left(z_{\sigma(1)}\right) \ldots \phi_{A_{\sigma(n)}}\left(z_{\sigma(n)}\right)\right| 0\right\rangle=(-1)^{\varepsilon(\sigma)}\left\langle 0\left|\phi_{A_{1}}\left(z_{1}\right) \ldots \phi_{A_{n}}\left(z_{n}\right)\right| 0\right\rangle,
$$

for every permutation $\sigma ;(-1)^{\varepsilon(\sigma)}$ is the corresponding statistical factor: $\varepsilon(\sigma)=$ $\sum p_{a_{\sigma(i)}} p_{a_{\sigma(j)}}(\bmod 2)$ the sum being over all pairs of indices $i<j$ such that $\sigma(i)>$ $\sigma(j)$.

Proof. Set

$$
\rho_{n}:=\left(\prod_{k=1}^{n} z_{k}^{2}\right)\left(\prod_{1 \leqslant k<l \leqslant n} z_{k l}^{2}\right) .
$$

From Axioms $(F)$ and $(S L)$ it follows that for sufficiently large $N \in \mathbb{N}$ the formal series $\rho_{n}^{N} \phi_{A_{1}}\left(z_{1}\right) \ldots \phi_{A_{n}}\left(z_{n}\right) \Psi$ is $\mathbb{Z}_{2}$-symmetric Taylor series in the $z$ 's with coefficients in $\mathcal{D}$. Hence, $P\left(z_{1}, \ldots, z_{n}\right):=\rho_{n}^{N}\left\langle\Psi_{1} \mid \phi_{A_{1}}\left(z_{1}\right) \ldots \phi_{A_{n}}\left(z_{n}\right) \Psi_{2}\right\rangle$ is a complex Taylor series, and if $H \Psi_{1}=\epsilon_{1} \Psi_{1}$ and $H \Psi_{2}=\epsilon_{2} \Psi_{2}$ it satisfies, in addition, the Euler equation

$$
\sum_{k=1}^{n} z_{k} \cdot \partial_{z_{k}} P\left(z_{1}, \ldots, z_{n}\right)=\left(2 n^{2 N}(n-1)^{N}+\epsilon_{1}-\epsilon_{2}-\sum_{k=1}^{n} d_{k}\right) P\left(z_{1}, \ldots, z_{n}\right)
$$

as a consequence of the commutation relations (2.18). Therefore, $P\left(z_{1}, \ldots, z_{n}\right)$ is a polynomial and it is clear that its coefficients are linear combinations of scalar products of the type $\left\langle\Psi_{1} \mid \phi_{A_{1}\left\{k_{1}, m_{1}, \sigma_{1}\right\}} \ldots \phi_{A_{n}\left\{k_{n}, m_{1}, \sigma_{1}\right\}} \Psi_{2}\right\rangle$. Because of Proposition 3.2 (b) we find that the series $\rho_{n}^{N}\left\langle\Psi_{1} \mid \phi_{A_{1}}\left(z_{1}\right) \ldots \phi_{A_{n}}\left(z_{n}\right) \Psi_{2}\right\rangle$ is a polynomial for all $\Psi_{1}, \Psi_{2} \in \mathcal{D}$ for $N \in \mathbb{N}$ sufficiently large.

We now divide by $\rho_{n}^{N}$ in the space $\mathbb{C} \llbracket z_{1} \rrbracket_{z_{1}^{2}} \ldots \llbracket z_{n} \rrbracket_{z_{n}^{2}}$ which contains $\left\langle\Psi_{1}\right|$ $\left.\phi_{A_{1}}\left(z_{1}\right) \ldots \phi_{A_{n}}\left(z_{n}\right) \Psi_{2}\right\rangle$, because of the property (3.5). (The inverse series 
of $\rho_{n}^{N}$ is obtained in $\mathbb{C} \llbracket z_{1} \rrbracket_{z_{1}^{2}} \ldots \llbracket z_{n} \rrbracket_{z_{n}^{2}}$ by expanding every factor $\left(z_{k l}^{2}\right)^{-N}=$ $\left(\left(z_{k}-z_{l}\right)^{2}\right)^{-N}$, for $1 \leqslant k<l \leqslant n$, in Taylor series in $z_{l}$ around $z_{l}=0$ - see Proposition $3.1(c))$.

Thus the domain of absolute convergence of the formal series $\left\langle\Psi_{1}\right| \phi_{A_{1}}\left(z_{1}\right)$ $\left.\ldots \phi_{A_{n}}\left(z_{n}\right) \Psi_{2}\right\rangle$ coincides with the domain of absolute convergence of the above expanded "propagators" $\left(z_{k l}^{2}\right)^{-N}$, which contains $\mathbb{U}_{n}^{<}$.

The covariance law (3.7) and the $\mathbb{Z}_{2}{ }^{-}$-symmetry (3.8) follow from the uniqueness of the analytic continuation.

Remark 3.3. The domain $T_{+}$can be characterized as the connected component of $z=0$ of the subset $\left\{z \in \mathbb{C}: \frac{\left(z-z^{*}\right)^{2}}{\left(z^{*}\right)^{2}} \equiv 1-2 z \cdot \bar{z}+\left|z^{2}\right|^{2} \neq 0\right\}$. Note that due to the axiom $(*)$ the product of $\phi_{A}(z)$ with its conjugate $\phi_{A}(z)^{*}$ will be singular for $\left(z-z^{*}\right)^{2}=0$.

\subsection{Ellipticity of the finite temperature correlation functions}

To study the thermal correlation functions it is convenient to use the compact picture representation of the GCI fields. Therefore, we begin with stating the basic properties of the compact picture formal series which are analogous to those of the formal power series of the previous subsection.

Denote by $V \llbracket e^{ \pm \pi i \zeta}, u \rrbracket$ the space of infinite formal Fourier series

$$
v(\zeta, u)=\sum_{\nu \in \frac{1}{2} \mathbb{Z}} \sum_{m=0}^{\infty} \sum_{\sigma} v_{\nu, m, \sigma} e^{-2 \pi i \nu \zeta} h_{\sigma}^{(m)}(u)
$$

with coefficients in $V$. It is a module over the algebra $\mathbb{C}\left[e^{ \pm \pi i \zeta}, u\right]$ of Fourier polynomials (the space of finite complex series of type (3.11)). When $\nu$ in Eq. (3.11) runs over $\mathbb{Z}$ the resulting space of series will be denoted by $V \llbracket e^{ \pm 2 \pi i \zeta}, u \rrbracket$. In the product $f(\zeta, u) v(\zeta, u)$ of $f(\zeta, u) \in \mathbb{C}\left[e^{ \pm \pi i \zeta}, u\right]$ and $v(\zeta, u) \in V \llbracket e^{ \pm \pi i \zeta}, u \rrbracket$ the harmonic polynomials $h_{\sigma}^{(m)}(u)$ are treated as spanning the algebra $\mathbb{C} \llbracket u \rrbracket$ of polynomial functions over the unit sphere $\mathbb{S}^{D-1}(\ni u)$. The space of $n$-point formal series, denoted by $V \llbracket e^{ \pm \pi i \zeta_{1}}, u_{1} ; \ldots ; e^{ \pm \pi i \zeta_{n}}, u_{n} \rrbracket$, is a module over the algebra $\mathbb{C}\left[e^{ \pm \pi i \zeta_{1}}, u_{1} ; \ldots\right.$; $\left.e^{ \pm \pi i \zeta_{n}}, u_{n}\right]$. Following the line of arguments of the previous subsection we need a compact picture analogue of the localized space $V \llbracket z \rrbracket_{z^{2}}$. Observing that the basic terms in (3.11) can be represented as:

$$
e^{-2 \pi i \nu \zeta} h_{\sigma}^{(m)}(u)=\left(z^{2}\right)^{n} h_{\sigma}^{(m)}(z) \quad \text { for } \quad z=e^{2 \pi i \zeta} u \quad \text { and } \quad \nu=-\frac{n+m}{2}
$$

we are led to introduce the space $V \llbracket e^{ \pm \pi i \zeta}, u \rrbracket_{+}$defined as containing those series (3.11) for which there exists $N \in \mathbb{N}$ such that $v_{\nu, m, \sigma}=0$ if $\nu+m>N$ (thus excluding arbitrary large powers of $z^{2}$ in the $z$-picture). It then follows from (3.12) that $v(\zeta, u) \in V \llbracket e^{ \pm \pi i \zeta}, u \rrbracket_{+}$iff $v(\zeta, u)=e^{-\pi i N \zeta} v^{\prime}\left(e^{2 \pi i \zeta} u\right)$ for some $N \in \mathbb{N}$ and 
$v^{\prime}(z) \in V \llbracket z \rrbracket$. We conclude, using Proposition 3.1, that $\mathbb{C} \llbracket e^{ \pm \pi i \zeta}, u \rrbracket+$ is a complex algebra and $V \llbracket e^{ \pm \pi i \zeta}, u \rrbracket_{+}$is a module over this algebra with no zero divisors.

Recall that $4 \sin \pi \zeta_{+} \sin \pi \zeta_{-}$, defined by Eqs. (1.11) and (1.12), the compact picture analogue of the interval $z_{12}^{2}$, is a Fourier polynomial belonging to $\mathbb{C}\left[e^{ \pm 2 \pi i \zeta_{1}}, u_{1}\right.$; $e^{ \pm 2 \pi i \zeta_{2}}, u_{2}$ ] (i.e., the space of series containing just integer powers of $e^{ \pm 2 \pi i \zeta_{k}}$ ). We shall now introduce an elliptic version of the compact picture interval:

$$
\begin{aligned}
& \Theta\left(\zeta_{1}, u_{1} ; \zeta_{2}, u_{2} ; \tau\right):=e^{-\frac{\pi i \tau}{2}} \vartheta_{11}\left(\zeta_{+}, \tau\right) \vartheta_{11}\left(\zeta_{-}, \tau\right)= \\
& \quad=4 \sin \pi \zeta_{+} \sin \pi \zeta_{-}-4\left(\sin 3 \pi \zeta_{+} \sin \pi \zeta_{-}+\sin \pi \zeta_{+} \sin 3 \pi \zeta_{-}\right) e^{2 \pi i \tau}+\ldots
\end{aligned}
$$

where $\vartheta_{11}(\zeta, \tau)$ are the Jacobi $\vartheta$-function (A.24). Having $n$ compact picture points $\left(\zeta_{1}, u_{1}\right) \ldots\left(\zeta_{n}, u_{n}\right)\left(\in \mathbb{R} \times \mathbb{S}^{D-1}\right)$ we introduce the shorthand notation:

$$
\Theta_{j k}:=\Theta\left(\zeta_{j}, u_{j} ; \zeta_{k}, u_{k} ; \tau\right), \quad \Omega\left(\zeta_{1}, u_{1} ; \ldots ; \zeta_{n}, u_{n}\right):=\prod_{1 \leqslant j<k \leqslant n} \Theta_{j k} .
$$

Each term of the series (3.13) and (3.14) is a function of the coordinate differences $\zeta_{j k}=\zeta_{j}-\zeta_{k}$; hence, we can write $\Theta\left(\zeta_{12} ; u_{1}, u_{2}\right)$ and $\Omega\left(\zeta_{12}, \ldots, \zeta_{n-1} ; u_{1}, \ldots, u_{n}\right)$ treating them, however, as series in different spaces.

\section{Proposition 3.4.}

(a) $\Theta_{12}$ is a formal series belonging to $\mathbb{C}\left[e^{ \pm 2 \pi i \zeta_{1}}, u_{1} ; e^{ \pm 2 \pi i \zeta_{2}}, u_{2}\right] \llbracket q \rrbracket\left(q=e^{2 \pi i \tau}\right)$, i.e., $\Theta_{12}$ is a Taylor series in $q$ with coefficients which are Fourier polynomials belonging to $\mathbb{C}\left[e^{ \pm 2 \pi i \zeta_{1}}, u_{1} ; e^{ \pm 2 \pi i \zeta_{2}}, u_{2}\right]$. Moreover, $\Theta_{12}$ is symmetric, $\Theta_{12}=$ $\Theta_{21}$, and it is divisible by $\sin \pi \zeta_{+} \sin \pi \zeta_{-}$:

$$
\begin{aligned}
\Theta_{12} & =4 \sin \pi \zeta_{+} \sin \pi \zeta_{-}\left(1+q \Theta_{12}^{\prime}\right)= \\
& =4 \sin \pi \zeta_{+} \sin \pi \zeta_{-}\left\{1-2\left(1+2 \cos 2 \pi \zeta_{12} \cos 2 \pi \alpha\right) q+\ldots\right\},
\end{aligned}
$$

where $\Theta_{12}^{\prime}$ is again a series belonging to $\mathbb{C}\left[e^{ \pm 2 \pi i \zeta_{1}}, u_{1} ; e^{ \pm 2 \pi i \zeta_{2}}, u_{2}\right] \llbracket q \rrbracket$.

(b) The formal series $\Theta_{12}$ has inverse:

$$
\Theta_{12}^{-1} \in \mathbb{C} \llbracket e^{ \pm 2 \pi i \zeta_{1}}, u_{1} \rrbracket_{+} \llbracket e^{ \pm 2 \pi i \zeta_{2}}, u_{2} \rrbracket_{+} \llbracket q \rrbracket \quad\left(\Theta_{12} \Theta_{12}^{-1}=1\right) .
$$

The series $\Theta_{12}^{-1}$ is absolutely convergent in the domain $0<-\operatorname{Im} \zeta_{12}<\operatorname{Im} \tau$.

(c) $\Omega\left(\zeta_{1}, u_{1} ; \ldots ; \zeta_{k}+\tau, u_{k} ; \ldots ; \zeta_{n}, u_{n}\right)$ is a symmetric formal series belonging to $\mathbb{C}\left[e^{ \pm 2 \pi i \zeta_{1}}, u_{1} ; \ldots ; e^{ \pm 2 \pi i \zeta_{n}}, u_{n}\right] \llbracket q \rrbracket$ (i.e., $\Omega\left(\zeta_{1}, u_{1} ; \ldots ; \zeta_{n}, u_{n}\right)=\Omega\left(\zeta_{\sigma_{1}}, u_{\sigma_{1}} ; \ldots ;\right.$ $\left.\left.\zeta_{\sigma_{n}}, u_{\sigma_{n}}\right)\right)$. As a series in the conformal time differences $\zeta_{j+1}, \Omega\left(\zeta_{12}, \ldots\right.$, $\left.\zeta_{n-1 n} ; u_{1}, \ldots, u_{n}\right)$ satisfies the property

$$
\begin{aligned}
& \Omega\left(\zeta_{12}+\lambda_{1} \tau, \ldots, \zeta_{n-1 n}+\lambda_{n-1} \tau ; u_{1}, \ldots, u_{n}\right)= \\
& =\exp \left\{-2 \pi i\left(\sum_{j, k=1}^{m-1} \lambda_{j} A_{j k}^{(n)} \lambda_{k}\right) \tau-4 \pi i \sum_{j, k=1}^{m-1} \lambda_{j} A_{j k}^{(n)} \zeta_{k k+1}\right\} \times \\
& \quad \times \Omega\left(\zeta_{12}, \ldots, \zeta_{n-1} ; u_{1}, \ldots, u_{n}\right)
\end{aligned}
$$


for all $\left(\lambda_{1}, \ldots, \lambda_{n-1}\right) \in \mathbb{Z}^{n-1}$, where $A_{j k}^{(n)}=A_{k j}^{(n)}$ are fixed integer constants (depending just on their indices) and the symmetric matrix $\left\{A_{j k}^{(n)}\right\}_{j, k=1}^{n-1}$ is positive definite.

(d) Let $R$ be a commutative complex algebra. The space of Taylor series $F\left(\zeta_{12}, \ldots\right.$, $\left.\zeta_{n-1 n} ; \tau\right)$ in $q^{\frac{1}{2}}$ which have polynomial coefficients belonging to $R\left[e^{ \pm \pi i \zeta_{12}}, \ldots\right.$, $\left.e^{ \pm \pi i \zeta_{n-1 n}}\right]$ and obey for all $\lambda_{1}, \ldots, \lambda_{n-1} \in \mathbb{Z}$ the properties

$$
\begin{aligned}
& F\left(\zeta_{12}+\lambda_{1}, \ldots, \zeta_{n-1 n}+\lambda_{n-1} ; \tau\right)=(-1)^{\sum_{k=1}^{n-1} \lambda_{k} \varepsilon_{k}^{(1)}} F\left(\zeta_{12}, \ldots, \zeta_{n-1} ; \tau\right), \\
& F\left(\zeta_{12}+\lambda_{1} \tau, \ldots, \zeta_{n-1}+\lambda_{n-1} \tau ; \tau\right)=(-1)^{\sum_{k=1}^{n-1} \lambda_{k} \varepsilon_{k}^{(\tau)}} \times \\
& \quad \times \exp \left\{-2 \pi i N\left(\sum_{j, k=1}^{m-1} \lambda_{j} A_{j k}^{(n)} \lambda_{k}\right) \tau-4 \pi i N \sum_{j, k=1}^{m-1} \lambda_{j} A_{j k}^{(n)} \zeta_{k k+1}\right\} \times \\
& \quad \times F\left(\zeta_{12}, \ldots, \zeta_{n-1 n} ; \tau\right),
\end{aligned}
$$

where $\varepsilon_{k}^{(1)}, \varepsilon_{k}^{(\tau)}=0,1(k=1, \ldots, n-1), N \in \mathbb{N}$ and $\left\{A_{j k}^{(n)}\right\}_{j, k=1}^{n-1}$ is integral positive matrix, is a finitely generated module over $R \llbracket q^{\frac{1}{2}} \rrbracket$. In other words, there exists a finite number of fixed (complex) series $F_{c}^{(N)}\left(\zeta_{12}, \ldots, \zeta_{n-1} ; \tau\right), c=$ $1, \ldots, C_{N}$, obeying the properties (3.18) and (3.19) and such that $F\left(\zeta_{12}, \ldots\right.$, $\left.\zeta_{n-1 n} ; \tau\right)=\sum_{c=1}^{C_{N}} G_{c}(\tau) F_{c}^{(N)}\left(\zeta_{12}, \ldots, \zeta_{n-1 n} ; \tau\right)$ for $G_{c}(\tau) \in R \llbracket q^{\frac{1}{2}} \rrbracket$. Moreover the basic series $F_{c}^{(N)}\left(\zeta_{12}, \ldots, \zeta_{n-1} ; \tau\right)$ can be chosen absolutely convergent to analytic functions for $\operatorname{Im} \tau>0$ and $\zeta_{k k+1} \in \mathbb{C}(k=1, \ldots, n-1)$. If we have a multicomponent series $F_{A_{1} \ldots A_{n}}\left(\zeta_{12}, \ldots, \zeta_{n-1 n} ; \tau\right)$ of the above type which, in addition, is $\mathbb{Z}_{2}$-symmetric (in the sense of $E q$. (3.8)) then we can expand it in a finite $R \llbracket q^{\frac{1}{2}} \rrbracket$-linear combination of $\mathbb{Z}_{2}$-symmetric basic series $F_{A_{1} \ldots A_{n} ; c}^{(N)}\left(\zeta_{12}\right.$, $\left.\ldots, \zeta_{n-1 n} ; \tau\right)$.

The proof of Proposition 3.4 is straightforward. We present it in Appendix B. Let us note that taking the ratios

$$
E_{c}^{(N)}\left(\zeta_{12}, \ldots, \zeta_{n-1 n} ; u_{1}, \ldots, u_{n} ; \tau\right):=\frac{F_{c}^{(N)}\left(\zeta_{12}, \ldots, \zeta_{n-1 n} ; \tau\right)}{\Omega\left(\zeta_{12}, \ldots, \zeta_{n-1 n} ; u_{1}, \ldots, u_{n}\right)^{N}}
$$

for $c=1, \ldots, C_{N}$, using the basic systems in Proposition $3.4(d)$, we obtain systems of formal series (for $N \in \mathbb{N}$ ) belonging to $\mathbb{C} \llbracket e^{ \pm 2 \pi i \zeta_{1}}, u_{1} \rrbracket_{+} \ldots \llbracket e^{ \pm 2 \pi i \zeta_{n}}, u_{n} \rrbracket+\llbracket q \rrbracket$ which are absolutely convergent in the domain $0<-\operatorname{Im} \zeta_{j k}<\operatorname{Im} \tau$ for $1 \leqslant j<k \leqslant$ $n$ to elliptic functions in every $\zeta_{k}$ (Proposition $3.4(b)$ and $(c)$ ):

$$
\begin{aligned}
& E_{c}^{(N)}\left(\zeta_{12}+\lambda_{1}, \ldots, \zeta_{n-1 n}+\lambda_{n-1} ; \tau\right)=(-1)^{\sum_{k=1}^{n-1} \lambda_{k} \varepsilon_{k}^{(1)}} E_{c}^{(N)}\left(\zeta_{12}, \ldots, \zeta_{n-1 n} ; \tau\right), \\
& E_{c}^{(N)}\left(\zeta_{12}+\lambda_{1} \tau, \ldots, \zeta_{n-1}+\lambda_{n-1} \tau ; \tau\right)=(-1)^{\sum_{k=1}^{n-1} \lambda_{k} \varepsilon_{k}^{(\tau)}} E_{c}^{(N)}\left(\zeta_{12}, \ldots, \zeta_{n-1} ; \tau\right) .
\end{aligned}
$$


The finite temperature correlation functions can be written as linear combinations of such ratios with coefficients that are $\tau$-dependent spherical functions (or, at least, formal Fourier series) in $u_{k}$.

We are now ready to find out the general structure of the Gibbs (thermal) correlation functions. When one considers the thermodynamic properties of quantum fields additional assumptions are always needed (see footnote 1). In our framework we impose the minimal assumption that the conformal Hamiltonian $H$ has finite dimensional eigenspaces. This makes it possible to introduce the partition function $Z(\tau)$, and the thermal mean values $\left\langle\phi_{A_{1} ; \nu_{1}, m_{1}, \sigma_{1}} \ldots \phi_{A_{k} ; \nu_{k}, m_{k}, \sigma_{k}}\right\rangle_{q}\left(q=e^{2 \pi i \tau}\right)$ of products of compact picture modes $\phi_{A ; \nu, m, \sigma}$ of fields $\phi_{A}(\zeta, u)$ as formal power series in $q^{\frac{1}{2}}$,

$$
\begin{gathered}
Z(\tau)=\operatorname{tr}_{\mathcal{D}}\left(q^{H}\right)=\sum_{j=0}^{\infty} \sum_{\sigma}\left\langle\Psi_{j \sigma} \mid \Psi_{j \sigma}\right\rangle q^{\frac{j}{2}} \\
\left\langle\phi_{A_{1} ; \nu_{1}, m_{1}, \sigma_{1}} \ldots \phi_{A_{k} ; \nu_{k}, m_{k}, \sigma_{k}}\right\rangle_{q}:=\frac{1}{Z(\tau)} \operatorname{tr}_{\mathcal{D}}\left\{\phi_{A_{1} ; \nu_{1}, m_{1}, \sigma_{1}} \ldots \phi_{A_{k} ; \nu_{k}, m_{k}, \sigma_{k}} q^{H}\right\}= \\
=\frac{1}{Z(\tau)} \sum_{j=0}^{\infty} \sum_{\sigma}\left\langle\Psi_{j \sigma} \mid \phi_{A_{1} ; \nu_{1}, m_{1}, \sigma_{1}} \ldots \phi_{A_{k} ; \nu_{k}, m_{k}, \sigma_{k}} \Psi_{j \sigma}\right\rangle q^{\frac{j}{2}}
\end{gathered}
$$

where $\left\{\Psi_{j \sigma}\right\}_{j \sigma}$ is an orthonormal basis in the Hilbert state space consisting of eigenvectors of $H, H \Psi_{j \sigma}=\frac{j}{2} \Psi_{j \sigma}$. (Note that $\mathcal{D}=\operatorname{Span}_{\mathbb{C}}\left\{\Psi_{j \sigma}\right\}_{j \sigma}$ because of Proposition $3.2(c)$; note also that the series of $Z(\tau)$ has a leading term 1 so that it is invertible in $\mathbb{C} \llbracket q^{\frac{1}{2}} \rrbracket$-see Fact B.1.) The cyclic property of the traces over each (finite dimensional) eigenspace of $H$ will imply the KMS property:

$$
\begin{gathered}
\left\langle\phi_{A_{1} ; \nu_{1}, m_{1}, \sigma_{1}} \ldots \phi_{A_{n} ; \nu_{k}, m_{k}, \sigma_{k}}\right\rangle_{q}= \\
=\left\langle\phi_{A_{2} ; \nu_{2}, m_{2}, \sigma_{2}} \ldots \phi_{A_{n} ; \nu_{k}, m_{k}, \sigma_{k}} q^{H} \phi_{A_{1} ; \nu_{1}, m_{1}, \sigma_{1}} q^{-H}\right\rangle_{q}= \\
=q^{-\nu_{1}}\left\langle\phi_{A_{2} ; \nu_{2}, m_{2}, \sigma_{2}} \ldots \phi_{A_{n} ; \nu_{k}, m_{k}, \sigma_{k}} \phi_{A_{1} ; \nu_{1}, m_{1}, \sigma_{1}}\right\rangle_{q}
\end{gathered}
$$

(according to Eq. (2.27)) as an equality in $\mathbb{C} \llbracket q^{\frac{1}{2}} \rrbracket$. Summing then over all triples $\nu_{l}, m_{l}, \sigma_{l}$ in the corresponding expansions of $\phi_{A_{l}}\left(\zeta_{l}, u_{l}\right)$ by $(2.25)$ we obtain the KMS equation

$$
\begin{gathered}
\left\langle\phi_{A_{1}}\left(\zeta_{1}, u_{1}\right) \ldots \phi_{A_{n}}\left(\zeta_{n}, u_{n}\right)\right\rangle_{q}= \\
=\left\langle\phi_{A_{2}}\left(\zeta_{2}, u_{2}\right) \ldots \phi_{A_{n}}\left(\zeta_{n}, u_{n}\right) \phi_{A_{1}}\left(\zeta_{1}+\tau, u_{1}\right)\right\rangle_{q}
\end{gathered}
$$

as an equality of formal series belonging to $\mathbb{C} \llbracket q^{\frac{1}{2}} \rrbracket \llbracket e^{ \pm 2 \pi i \zeta_{1}}, u_{1} ; \ldots ; e^{ \pm 2 \pi i \zeta_{n}}, u_{n} \rrbracket$.

On the other hand, one can perform the sum in the trace $\operatorname{tr}_{\mathcal{D}}\left\{\phi_{A_{1}}\left(\zeta_{1}, u_{1}\right) \ldots\right.$ $\left.\phi_{A_{n}}\left(\zeta_{n}, u_{n}\right) q^{H}\right\}$ first over the fields' modes and then over the energy levels (taking the sum in the powers of $\left.q^{\frac{1}{2}}\right)$,

$$
\begin{gathered}
\operatorname{tr}_{\mathcal{D}}\left\{\phi_{A_{1}}\left(\zeta_{1}, u_{1}\right) \ldots \phi_{A_{n}}\left(\zeta_{n}, u_{n}\right) q^{H}\right\}= \\
=\sum_{j=0}^{\infty} \sum_{\sigma}\left\langle\Psi_{j \sigma} \mid \phi_{A_{1}}\left(\zeta_{1}, u_{1}\right) \ldots \phi_{A_{n}}\left(\zeta_{n}, u_{n}\right) \Psi_{j \sigma}\right\rangle q^{\frac{j}{2}} ;
\end{gathered}
$$


this gives a meaning to Eq. (3.25) as an equality in the space $C \llbracket e^{ \pm 2 \pi i \zeta_{1}}, u_{1} \rrbracket_{+} \ldots$ $\llbracket e^{ \pm 2 \pi i \zeta_{n}}, u_{n} \rrbracket_{+} \llbracket q^{\frac{1}{2}} \rrbracket$ (according to Eq. (3.2)). Now, if we multiply both sides of (3.25) by $\Omega\left(\zeta_{12}, \ldots, \zeta_{n-1 n} ; u_{1}, \ldots, u_{n}\right)^{N}$ for some $N \gg 0$, setting

$$
F_{A_{1} \ldots A_{n}}\left(\zeta_{12}, \ldots, \zeta_{n-1 n} ; u_{1}, \ldots, u_{n} ; \tau\right):=\Omega^{N}\left\langle\phi_{A_{1}}\left(\zeta_{1}, u_{1}\right) \ldots \phi_{A_{n}}\left(\zeta_{n}, u_{n}\right)\right\rangle_{q},
$$

we find that $F_{A_{1} \ldots A_{n}}$ are symmetric formal Fourier series belonging to $\mathbb{C}\left[e^{ \pm \pi i \zeta_{1}}, u_{1}\right.$; $\ldots ; e^{ \pm \pi i \zeta_{n}}, u_{n} \rrbracket \llbracket q^{\frac{1}{2}} \rrbracket$ (i.e., with symmetric polynomial coefficients multiplying each power of $\left.q^{\frac{1}{2}}\right)$ and they also obey the properties (3.18) and (3.19) with $\varepsilon_{k}^{(1)}=\varepsilon_{k}^{(2)}=$ $\sum_{\ell=k+1}^{n} p_{\ell}$ for $k=1, \ldots, n-1$ ( $p_{\ell}$ being the fermion parities). The first statement is verified by using the rationality (Theorem 3.3) and Eq. (3.15), while the second uses Proposition $3.4(c)$ and the KMS equation (3.25) combined with the $\mathbb{Z}_{2}-$ symmetry (3.8). Thus, we can apply Proposition $3.4(d)$ (with $R$, the algebra $\mathbb{C}\left[u_{1}\right.$, $\left.\ldots, u_{n}\right]$ of harmonic polynomials in $u_{1}, \ldots, u_{n}$ restricted to $\left.\mathbb{S}^{D-1}\right)$ obtaining the expansion

$$
\begin{gathered}
\left\langle\phi_{A_{1}}\left(\zeta_{1}, u_{1}\right) \ldots \phi_{A_{n}}\left(\zeta_{n}, u_{n}\right)\right\rangle_{q}= \\
=\sum_{c=1}^{C_{N}} G_{c ; A_{1} \ldots A_{n}}\left(u_{1}, \ldots, u_{n} ; \tau\right) E_{c}^{(N)}\left(\zeta_{12}, \ldots, \zeta_{n-1} ; u_{1}, \ldots, u_{n} ; \tau\right)
\end{gathered}
$$

in the basic elliptic functions of the system (3.20). The coefficients $G_{c ; A_{1} \ldots A_{n}}\left(u_{1}\right.$, $\left.\ldots, u_{n} ; \tau\right)$ are Taylor series in $q^{\frac{1}{2}}$ with symmetric polynomial coefficients in $u_{1}, \ldots, u_{n} \in \mathbb{S}^{D-1}$ (i.e., $G_{c ; A_{1} \ldots A_{n}} \in \mathbb{C}\left[u_{1}, \ldots, u_{n}\right] \llbracket q^{\frac{1}{2}} \rrbracket$ ).

We thus end up with the following result.

Theorem 3.5. Under the assumptions of Sect. 2 and the additional condition that the conformal Hamiltonian $H$ has finite dimensional eigenspaces every $n$-point thermal correlation function $\left\langle\phi_{A_{1}}\left(\zeta_{1}, u_{1}\right) \ldots \phi_{A_{n}}\left(\zeta_{n}, u_{n}\right)\right\rangle_{q}$ admits a formal series representation of type (3.28) where $G_{c ; A_{1} \ldots A_{n}}\left(u_{1}, \ldots, u_{n} ; \tau\right)$ are Taylor series in $q^{\frac{1}{2}}$ with symmetric (harmonic) polynomial coefficients in $u_{1}, \ldots, u_{n} \in \mathbb{S}^{D-1}$ and $E_{c}^{(N)}\left(\zeta_{12}, \ldots, \zeta_{n-1 n} ; u_{1}, \ldots, u_{n} ; \tau\right)\left(c=1, \ldots, C_{N}\right)$ are some fixed series which belong to $\mathbb{C} \llbracket e^{ \pm 2 \pi i \zeta_{1}}, u_{1} \rrbracket_{+} \ldots \llbracket e^{ \pm 2 \pi i \zeta_{n}}, u_{n} \rrbracket_{+} \llbracket q^{\frac{1}{2}} \rrbracket$ and are absolutely convergent in the domain

$$
\left\{\left(\zeta_{1}, u_{1} ; \ldots ; \zeta_{n}, u_{n} ; \tau\right) \in\left(\mathbb{C} \times \mathbb{S}^{D-1}\right)^{n} \times \mathfrak{H}: 0<-\operatorname{Im} \zeta_{j k}<\operatorname{Im} \tau(1 \leqslant j<k \leqslant n)\right\}
$$

to $\mathbb{Z}_{2}$-symmetric (in the sense of Eq. (3.8)) meromorphic functions over $\left(\mathbb{C} \times \mathbb{S}^{D-1}\right)^{n} \times \mathfrak{H}$. Moreover, the resulting functions are doubly periodic (resp., antiperiodic) in $\zeta_{m}$ with periods 1 and $\tau$ if $\phi_{A_{m}}$ is a bosonic (resp., fermionic) field, for $m=1, \ldots, n$.

The problem of summability of the angular coefficients $G_{c ; A_{1} \ldots A_{n}}\left(u_{1}, \ldots, u_{n} ; \tau\right)$ is still open. Let us note first that if we exchange the order of summation in $G_{c ; A_{1} \ldots A_{n}}$ first summing in the powers of $q^{\frac{1}{2}}$ and then over the harmonic polynomials in $u_{k}$ we will obtain $G_{c ; A_{1} \ldots A_{n}}$ as elements of the space $\mathbb{C} \llbracket q^{\frac{1}{2}} \rrbracket \llbracket u_{1}, \ldots, u_{n} \rrbracket$, i.e., the space of infinite harmonic power series in $u_{1}, \ldots, u_{n}$ with coefficients in 
$\mathbb{C} \llbracket q^{\frac{1}{2}} \rrbracket$. These coefficients can be expressed by a finite set of series in $q^{\frac{1}{2}}$ of type (3.22) and (3.23), using the operations of summation and multiplication, since all above considerations have been made in a purely algebraic setting. Thus, if we assume that the partition function and all thermal mean values of products of fields' modes are absolutely convergent series for $\left|q^{\frac{1}{2}}\right|<1$ we obtain $G_{c ; A_{1} \ldots A_{n}}$ as infinite formal Fourier series in $\left(u_{1}, \ldots, u_{n}\right) \in \mathbb{S}^{(D-1) n}$ whose convergence should be further assumed in order to end up with elliptic finite temperature correlation functions. Let us conclude this discussion with the remark that in a chiral conformal QFT (which is, essentially, an 1-dimensional theory) there are no angular variables $u$ so that Theorem 3.5 actually states the existence of the finite temperature correlation functions as elliptic functions under the assumptions of convergence of the partition function and the thermal mean values of the product of the fields' modes.

Corollary 3.6. In the assumptions of Theorem 3.5 let the finite temperature correlation functions absolutely converge in the domain (3.29) to meromorphic functions. Then these function are elliptic of the type of $E_{c}^{(N)}$ appearing in the representation (3.27).

In the following section we shall calculate the finite temperature correlation functions in free fields' models and will see that they satisfy the above assumptions and are indeed elliptic functions.

\section{Free Field Models}

\subsection{General properties of thermal correlation functions of free fields}

A generalized free field is defined as a Fock space representation of the HeisenbergDirac algebra with generators $\phi_{A\{n, m, \sigma\}}$ as in Eq. $(2.13)(A=1, \ldots, I)$. It is completely determined by its 2 -point function

$$
\left\langle 0\left|\phi_{A}(z) \stackrel{*}{\phi}_{B}(w)\right| 0\right\rangle=\iota_{z, w} \mathcal{W}_{A B}(z, w), \quad \mathcal{W}_{A B}(z, w)=\frac{Q_{A B}(z-w)}{\left[(z-w)^{2}\right]^{\mu_{A B}}},
$$

where $Q_{A B}(z)$ are polynomials and we recall that $\iota_{z, w}$ stands for the Taylor expansion of $\mathcal{W}_{A B}(z, w)$ in $w$ whose coefficients are rational functions in $z$. Note that the $\iota_{z, w}$ operation is the $z$-picture counterpart of the " $i 0\left(x^{0}-y^{0}\right)$ " prescription in Minkowski space which turns, for example, the rational function $\frac{1}{(x-y)^{2}}$, into the distribution $\frac{1}{(x-y)^{2}+i 0\left(x^{0}-y^{0}\right)}$. Then the generating function of the modes' (anti)commutation relations is

$$
\begin{aligned}
\phi_{A}(z) \stackrel{*}{\phi_{B}}(w)- & (-1)^{p_{A} p_{B}} \stackrel{*}{\phi}_{B}(w) \phi_{A}(z)=\iota_{z, w} \mathcal{W}_{A B}(z, w)-\iota_{w, z} \mathcal{W}_{A B}(z, w)= \\
& =\iota_{z, w} \mathcal{W}_{A B}(z, w)-(-1)^{p_{A} p_{B}} \iota_{w, z} \mathcal{W}_{B A}(w, z) .
\end{aligned}
$$


The annihilation operators are the modes $\phi_{A\{n, m, \sigma\}}$ with $n<0$. The Fock space is generated by the one particle state space $\mathcal{D}_{1}$ spanned by the vectors $\phi_{A\{n, m, \sigma\}}|0\rangle$ for $n \geqslant 0$ and its hermitian scalar product is determined by the contributions of the Laurent modes $\left\langle 0\left|\phi_{A\{n, m, \sigma\}} \phi_{B\{n, m, \sigma\}}^{*}\right| 0\right\rangle$ to the 2-point function (4.1). We will not assume, in general, that the inner product in $\mathcal{D}_{1}$ is positive definite. The rational two-point function $\mathcal{W}_{A B}(z, w)$ is, by assumption, conformally invariant with respect to a cocycle $\pi_{z}(g)_{A}^{B}$.

The partition function $\operatorname{tr}_{\mathcal{D}}\left(q^{H}\right)$ and the other traces below are understood as traces taken over some (pseudo)orthonormal basis of $\mathcal{D}$ consisting of eigenvectors of $H$ (as in Sect. 3). It is a Taylor series in $q^{\frac{1}{2}}$ which is always convergent for $q^{\frac{1}{2}}=e^{i \pi \tau}$ with $\operatorname{Im} \tau>0\left(\left|q^{\frac{1}{2}}\right|<1\right)$ since the degree of degeneracy of the conformal energy level $\frac{n}{2}$ in the 1-particle state space has an upper bound of the form $C_{1}\left(\begin{array}{c}n+C_{2} \\ D-1\end{array}\right)$ with some positive constants $C_{1,2}$. More specifically, due to the spin-statistics theorem (which follows from the rationality of (4.1)), the integer conformal energy levels $n$ in $\mathcal{D}_{1}$ should belong to the bosonic 1-particle subspace while the half-integer ones, $n-\frac{1}{2}$, belong to the fermionic subspace. Then, the partition function is determined by the dimensions of these energy spaces. Let us denote these dimensions by $d_{B}(n)$ and $d_{f}(n)$ (for the bosonic and fermionic 1-particle spaces of energies $n$ and $n-\frac{1}{2}$, respectively); then we will have

$$
Z(\tau):=\operatorname{tr}_{\mathcal{D}}\left(q^{H}\right)=\prod_{n=1}^{\infty} \frac{\left(1+q^{n-\frac{1}{2}}\right)^{d_{f}(n)}}{\left(1-q^{n}\right)^{d_{B}(n)}} .
$$

It is also easy to see that the temperature mean value (3.23) of the products of (compact picture) modes $\phi_{A ; \nu, m}(u)$ (see Eq. (2.25)), is absolutely convergent. Moreover, it is expressed by Wick theorem in terms of "1-" and "2-point" Gibbs expectation values $\left\langle\phi_{A ; \nu, m}(u)\right\rangle_{q}$ and $\left\langle\phi_{A ; \nu_{1}, m_{1}}\left(u_{1}\right) \stackrel{*}{\phi}_{B ; \nu_{2}, m_{2}}\left(u_{2}\right)\right\rangle_{q}$, where $\stackrel{*}{\phi}_{B ; \nu, m}(u)(=$ $\left.\sum_{\sigma} \stackrel{*}{\phi}_{B ; \nu, m, \sigma} h_{\sigma}^{(m)}(u)\right)$ are the modes of the conjugate field $\stackrel{*}{\phi}_{B}(\zeta, u)$ (see Eq. $(2.27)$ ). Combining the KMS property (3.24)

$$
\left\langle\phi_{A ; \nu_{1}, m_{1}}\left(u_{1}\right) \stackrel{*}{\phi}_{B ; \nu_{2}, m_{2}}\left(u_{2}\right)\right\rangle_{q}=q^{-\nu_{1}}\left\langle\stackrel{*}{\phi}_{B ; \nu_{2}, m_{2}}\left(u_{1}\right) \phi_{A ; \nu_{1}, m_{1}}\left(u_{2}\right)\right\rangle_{q},
$$

with the canonical (anti)commutation relations (4.2) of the modes we obtain

$$
\begin{gathered}
\left\langle\phi_{A ; \nu_{1}, m_{1}}\left(u_{1}\right) \stackrel{*}{\phi}_{B ; \nu_{2}, m_{2}}\left(u_{2}\right)\right\rangle_{q}= \\
=\frac{1}{1-(-1)^{p_{A} p_{B}} q^{\nu_{1}}}\left\langle 0\left|\left[\phi_{A ; \nu_{1}, m_{1}}\left(u_{1}\right), \stackrel{*}{\phi}_{B ; \nu_{2}, m_{2}}\left(u_{2}\right)\right]_{-(-1)^{p_{A} p_{B}}}\right| 0\right\rangle .
\end{gathered}
$$

Theorem 4.1. The series $\left\langle\phi_{A}\left(\zeta_{1}, u_{1}\right) \stackrel{*}{\phi}_{B}\left(\zeta_{2}, u_{2}\right)\right\rangle_{q}$ is absolutely convergent for $0<-\operatorname{Im} \zeta_{12}<\operatorname{Im} \tau$ to an elliptic function in $\zeta_{12}$. It can be written as a series

$$
\left\langle\phi_{A}\left(\zeta_{1}, u_{1}\right) \stackrel{*}{\phi_{B}}\left(\zeta_{2}, u_{2}\right)\right\rangle_{q}=\sum_{k=-\infty}^{\infty}(-1)^{k p_{A} p_{B}} W_{A B}\left(\zeta_{12}+k \tau ; u_{1}, u_{2}\right),
$$


absolutely convergent in the same domain; here $W_{A B}\left(\zeta_{12} ; u_{1}, u_{2}\right)$ is the meromorphic vacuum correlation function

$$
W_{A B}\left(\zeta_{12} ; u_{1}, u_{2}\right):=\left\langle 0\left|\phi_{A}\left(\zeta_{1}, u_{1}\right) \stackrel{\phi}{B}_{B}\left(\zeta_{2}, u_{2}\right)\right| 0\right\rangle
$$

The functions (4.6) are manifestly doubly periodic elliptic functions in $\zeta_{12}$.

Proof. First observe that $\stackrel{*}{\phi}_{B ; \nu, m}(u)|0\rangle=0$ if $\nu \geqslant 0$ (in accord with Proposition 3.2) and therefore, $\langle 0| \phi_{B ; \nu, m}(u)=0$ if $\nu \leqslant 0$. Thus, at most one term contributes to the (anti) commutator in the right hand side of (4.5) and in fact:

$$
\begin{aligned}
\langle 0| & {\left[\phi_{A ; \nu_{1}, m_{1}}\left(u_{1}\right), \stackrel{*}{\phi}_{B ; \nu_{2}, m_{2}}\left(u_{2}\right)\right]_{-(-1)^{p_{A} p_{B}}}|0\rangle=} \\
= & \theta_{\nu_{1}}\left\langle 0\left|\phi_{A ; \nu_{1}, m_{1}}\left(u_{1}\right) \stackrel{*}{\phi}_{B ; \nu_{2}, m_{2}}\left(u_{2}\right)\right| 0\right\rangle- \\
& -(-1)^{p_{A} p_{B}} \theta_{-\nu_{1}}\left\langle 0\left|\stackrel{*}{\phi}_{B ; \nu_{2}, m_{2}}\left(u_{2}\right) \phi_{A ; \nu_{1}, m_{1}}\left(u_{1}\right)\right| 0\right\rangle
\end{aligned}
$$

where $\theta_{s}$ is the characteristic function of the positive numbers $\left(\theta_{s}:=1\right.$ for $s>0$ and $\theta_{s}=0$ otherwise). Expanding for $\left|q^{\frac{1}{2}}\right|<1\left(q^{\frac{1}{2}}=e^{i \pi \tau}\right)$ the prefactor in the right hand side of (4.5), we find:

$$
\begin{aligned}
&\left\langle\phi_{A}\left(\zeta_{1}, u_{1}\right) \stackrel{*}{\phi}_{B}\left(\zeta_{2}, u_{2}\right)\right\rangle_{q}= \\
&=\sum_{\substack{\nu_{1}, m_{1} \\
\nu_{2}, m_{2}}}\left\langle\phi_{A ; \nu_{1}, m_{1}}\left(u_{1}\right) \stackrel{*}{\phi}_{B ; \nu_{2}, m_{2}}\left(u_{2}\right)\right\rangle_{q} e^{-2 \pi i\left(\nu_{1} \zeta_{1}+\nu_{2} \zeta_{2}\right)}= \\
&=\sum_{\nu, m_{1}, m_{2}} \sum_{k=0}^{\infty}\left(\theta_{\nu}(-1)^{k p_{A} p_{B}} q^{k \nu}\left\langle 0\left|\phi_{A ; \nu, m_{1}}\left(u_{1}\right) \stackrel{*}{\phi}_{B ;-\nu, m_{2}}\left(u_{2}\right)\right| 0\right\rangle+\right. \\
&\left.\quad+\theta_{-\nu}(-1)^{k p_{A} p_{B}} q^{-(k+1) \nu}\left\langle 0\left|\stackrel{*}{\phi}_{B ;-\nu, m_{2}}\left(u_{2}\right) \phi_{A ; \nu, m_{1}}\left(u_{1}\right)\right| 0\right\rangle\right) \times \\
& \times e^{-2 \pi i \nu \zeta_{12}} .
\end{aligned}
$$

If we first perform the sum over the indices $\nu, m_{1}, m_{2}$ in the right hand side of (4.8) we obtain (due to Theorem 3.3) the series expansion in Eq. (4.6): indeed, the first term in the sum gives $\sum_{k=0}^{\infty}(-1)^{k p_{A} p_{B}} W_{A B}\left(\zeta_{12}+k \tau ; u_{1}, u_{2}\right)$ while the second gives

$$
\begin{aligned}
& \sum_{k=1}^{\infty}(-1)^{(-k+1) p_{A} p_{B}} W_{B A}^{\prime}\left(-\zeta_{12}-k \tau ; u_{2}, u_{1}\right), \text { where } \\
& W_{B A}^{\prime}\left(\zeta_{12} ; u_{1}, u_{2}\right):=\left\langle 0\left|\stackrel{*}{\phi}_{B}\left(\zeta_{1}, u_{1}\right) \phi_{A}\left(\zeta_{2}, u_{2}\right)\right| 0\right\rangle,
\end{aligned}
$$

so we should further apply the symmetry property

$$
W_{A B}\left(\zeta_{12} ; u_{1}, u_{2}\right)=(-1)^{p_{A} p_{B}} W_{B A}^{\prime}\left(-\zeta_{12} ; u_{2}, u_{1}\right)
$$

The series (4.6) is absolutely convergent since its terms behave as

$$
\begin{gathered}
W_{A B}\left(\zeta_{12}+k \tau ; u_{1}, u_{2}\right) \sim q^{k d_{B}} e^{2 \pi i\left(d_{A} \zeta_{1}+d_{B} \zeta_{2}\right)} \mathcal{W}_{A B}\left(e^{2 \pi i \zeta_{1}} u_{1}, 0\right) \text { for } k \rightarrow \infty, \\
W_{A B}\left(\zeta_{12}+k \tau ; u_{1}, u_{2}\right) \sim q^{-k d_{A}} e^{2 \pi i\left(d_{A} \zeta_{1}+d_{B} \zeta_{2}\right)} \mathcal{W}_{A B}\left(0, e^{2 \pi i \zeta_{2}} u_{2}\right) \text { for } k \rightarrow-\infty
\end{gathered}
$$


The series of the finite temperature correlation function $\left\langle\phi_{A}\left(\zeta_{1}, u_{1}\right) \stackrel{*}{\phi_{B}}\left(\zeta_{2}, u_{2}\right)\right\rangle_{q}$ given by the first equality in (4.8) is also absolutely convergent for $0<-\operatorname{Im} \zeta_{12}$ $<\operatorname{Im} \tau$ since the series of $W_{A B}$ absolutely converges in this domain.

Remark 4.1. Let $N$ be a hermitian operator, commuting with the conformal Hamiltonian $H$ and such that

$$
\left[N, \stackrel{*}{\phi}_{A}(z)\right]=n_{A} \stackrel{*}{\phi}_{A}(z), \quad\left[N, \phi_{A}(z)\right]=-n_{A} \phi_{A}(z) .
$$

Then we can derive in the same way as above the following expression for the grand canonical correlation functions

$$
\begin{aligned}
\left\langle\phi_{A}\left(\zeta_{1}, u_{1}\right) \stackrel{*}{\phi}_{B}\left(\zeta_{1}, u_{1}\right)\right\rangle_{q, \mu}: & =\frac{\operatorname{tr}_{\mathcal{D}}\left(\phi_{A}\left(\zeta_{1}, u_{1}\right) \stackrel{*}{\phi}_{B}\left(\zeta_{2}, u_{2}\right) q^{H} e^{2 \pi i \mu N}\right)}{\operatorname{tr}_{\mathcal{D}}\left(q^{H} e^{2 \pi i \mu N}\right)}= \\
& =\sum_{k=-\infty}^{\infty} e^{\pi i k\left(2 \mu+p_{A} p_{B}\right)} W_{A B}\left(\zeta_{12}+k \tau ; u_{1}, u_{2}\right)
\end{aligned}
$$

for real $\mu$. (In the physical literature the grand canonical partition function is written as $\operatorname{tr}\left(e^{-\beta(H-\mu N)}\right)$ where $\beta$ is the inverse temperature and $\mu$ is the chemical potential.)

Remark 4.2. In the assumptions of Corollary 3.6 one can state that for the thermal 2-point function $\left\langle\phi_{A}\left(\zeta_{1}, u_{1}\right) \phi_{B}^{*}\left(\zeta_{2}, u_{2}\right)\right\rangle_{q}$, of an arbitrary field $\phi=$ $\left\{\phi_{A}\right\}$, the right hand side of Eq. (4.6) describes the most singular part in $\zeta_{12}$ since it comes from the most singular part of the operator product expansion of $\phi_{A}\left(\zeta_{1}, u_{1}\right) \stackrel{*}{\phi}_{B}\left(\zeta_{2}, u_{2}\right)$ (see Proposition 4.3 of $[29]$ ).

\subsection{Free scalar fields}

The generalized free neutral scalar field $\phi(z) \equiv \phi^{(d)}(z)$ of dimension $d$ is determined by the unique conformally invariant scalar 2-point function (2.28).

Many of the modes in the field expansion (2.13) are zero so that it is convenient to reduce the system of basic functions and actually, organize the field modes in a slightly different way.

Let us denote by $\phi_{-d-n}(z)$ the homogeneous operator-valued polynomial of degree $n \geqslant 0$ contributing to the Taylor part of the expansion (2.13) of $\phi(z)$. The polynomial $\phi_{-d-n}(\bar{z})^{*}$ obtained conjugating the coefficients of $\phi_{-d-n}(z)$ is denoted by

$$
\phi_{n+d}(z)=\phi_{-d-n}(\bar{z})^{*}
$$

$(n \geqslant 0)$. Due to Proposition 3.2 , the creation modes of the field are exactly $\left\{\phi_{-n-d}\right.$ : $n \geqslant 0\}$ so that the remaining nonzero field modes $\left\{\phi_{n+d}: n \geqslant 0\right\}$ annihilate the vacuum $|0\rangle$. Thus the field $\phi(z)$ is expanded in the above modes as follows:

$$
\phi(z)=\sum_{n=0}^{\infty} \phi_{-n-d}(z)+\sum_{n=0}^{\infty}\left(z^{2}\right)^{-n-d} \phi_{n+d}(z) .
$$


The commutation relation with the conformal Hamiltonian take the form

$$
\left[H, \phi_{n}(z)\right]=-n \phi_{n}(z) \quad(n \in \mathbb{Z}) .
$$

The vacuum matrix elements of products of field modes are derived from the 2-point function

$$
\left\langle 0\left|\phi(\bar{z})^{*} \phi(w)\right| 0\right\rangle=\frac{1}{\left(1-2 z \cdot w+z^{2} w^{2}\right)^{d}}=\sum_{n=0}^{\infty} \widetilde{C}_{n}^{d}(z, w),
$$

where $\widetilde{C}_{n}^{d}(z, w)$ are polynomials separately homogeneous in $z$ and $w$ of equal degrees $n$ with generating function

$$
\frac{1}{\left(1-2 z \cdot w \lambda+z^{2} w^{2} \lambda^{2}\right)^{d}}=\sum_{n=0}^{\infty} \widetilde{C}_{n}^{d}(z, w) \lambda^{n} .
$$

Then

$$
\left\langle 0\left|\phi_{m+d}(z) \phi_{-n-d}(w)\right| 0\right\rangle=\delta_{m, n} \widetilde{C}_{n}^{d}(z, w)
$$

for $m, n \geqslant 0$. Note that the polynomials $\widetilde{C}_{n}^{d}(z, w)$ are related to the Gegenbauer polynomials $C_{n}^{d}(t)$ (with generating function $\frac{1}{\left(1-2 t \lambda+\lambda^{2}\right)^{d}}=\sum_{n=0}^{\infty} C_{n}^{d}(t) \lambda^{n}$ ) by

$$
\widetilde{C}_{n}^{d}(z, w)=\left(z^{2} w^{2}\right)^{\frac{n}{2}} C_{n}^{d}\left(\frac{z \cdot w}{\left(z^{2} w^{2}\right)^{1 / 2}}\right) .
$$

In the real compact picture we set

$$
\phi(\zeta, u)=e^{2 \pi i d \zeta} \phi\left(e^{2 \pi i \zeta} u\right)=\sum_{\substack{n \in \mathbb{Z} \\|n| \geqslant d}} e^{-2 \pi i n \zeta} \phi_{-n}(u)
$$

as a formal Fourier series in $\zeta$. Taking into account the relation

$$
\left\langle 0\left|\phi\left(e^{2 \pi i \zeta_{1}} u_{1}\right) \phi\left(e^{2 \pi i \zeta_{2}} u_{2}\right)\right| 0\right\rangle=e^{2 \pi i d\left(\zeta_{1}+\zeta_{2}\right)}\left\langle 0\left|\phi\left(\zeta_{1}, u_{1}\right) \phi\left(\zeta_{2}, u_{2}\right)\right| 0\right\rangle
$$

we find

$$
\left\langle 0\left|\phi\left(\zeta_{1}, u_{1}\right) \phi\left(\zeta_{2}, u_{2}\right)\right| 0\right\rangle=\frac{(-1)^{d}}{4^{d} \sin ^{d} \pi \zeta_{+} \sin ^{d} \pi \zeta_{-}},
$$

where $\zeta_{ \pm}=\zeta_{12} \pm \alpha, \cos 2 \pi \alpha=u_{1} \cdot u_{2}$. Then Eq. (4.5) takes the form

$$
\left\langle\phi\left(\zeta_{1}, u_{1}\right) \phi\left(\zeta_{2}, u_{2}\right)\right\rangle_{q}=\sum_{k=-\infty}^{\infty} \frac{(-1)^{d}}{4^{d} \sin ^{d} \pi\left(\zeta_{+}+k \tau\right) \sin ^{d} \pi\left(\zeta_{-}+k \tau\right)} .
$$

For $d=1$ we obtain

$$
\left\langle\phi\left(\zeta_{1}, u_{1}\right) \phi\left(\zeta_{2}, u_{2}\right)\right\rangle_{q}=\frac{1}{4 \pi \sin 2 \pi \alpha}\left(p_{1}\left(\zeta_{+}, \tau\right)-p_{1}\left(\zeta_{-}, \tau\right)\right),
$$


where $p_{k}(\zeta, \tau)$ are written down in Appendix A (see (A.28)). Eq. (4.22) follows from the identity

$$
\frac{-1}{\sin \pi \zeta_{+} \sin \pi \zeta_{-}}=\frac{1}{\sin 2 \pi \alpha}\left(\operatorname{cotg} \pi \zeta_{+}-\operatorname{cotg} \pi \zeta_{-}\right)
$$

and (4.21). Note that the differences in (4.23) and (4.22) allows us to apply Eq. (A.28) and ensures the ellipticity (double periodicity) in $\zeta_{12}$ of the thermal correlation function.

Remark 4.3. The Gibbs 2-point function of the modes $\phi_{n}(u)$ in the latter example $(d=1)$

$$
\left\langle\phi_{-m}\left(u_{1}\right) \phi_{n}\left(u_{2}\right)\right\rangle_{q}=\delta_{m n} \frac{q^{n}}{1-q^{n}} \frac{\sin 2 \pi n \alpha}{\sin 2 \pi \alpha}
$$

(for $u_{1} \cdot u_{2}=\cos 2 \pi \alpha$ ), which can be derived directly from the canonical commutation relations and the KMS condition, yields the $q$-expansion of (4.22)

$\left\langle\phi\left(\zeta_{1}, u_{1}\right) \phi\left(\zeta_{2}, u_{2}\right)\right\rangle_{q}=\frac{-1}{4 \sin \pi \zeta_{+} \sin \pi \zeta_{-}}+2 \sum_{n=1}^{\infty} \frac{q^{n}}{1-q^{n}} \frac{\sin 2 \pi n \alpha}{\sin 2 \pi \alpha} \cos 2 \pi n \zeta_{12}$.

Comparing with (4.22) we deduce a similar expansion for $p_{1}$

$$
p_{1}(\zeta, \tau)=\pi \cot \pi \zeta+4 \pi \sum_{n=1}^{\infty} \frac{q^{n}}{1-q^{n}} \sin 2 \pi n \zeta .
$$

In the more general context of Remark 4.1, for a complex scalar field of dimension $1\left(\left\langle 0\left|\phi\left(z_{1}\right) \stackrel{*}{\phi}\left(z_{2}\right)\right| 0\right\rangle=\left(z_{12}^{2}\right)^{-1}\right)$ taking $N$ to be the charge operator (with $n=1$ in Eq. (4.10)), we find

$$
\left\langle\phi\left(\zeta_{1}, u_{1}\right) \stackrel{*}{\phi}\left(\zeta_{2}, u_{2}\right)\right\rangle_{q, \mu}=\frac{1}{4 \pi \sin 2 \pi \alpha}\left(p_{1}\left(\zeta_{+}, \tau, \mu\right)-p_{1}\left(\zeta_{-}, \tau, \mu\right)\right)
$$

for the more general functions $p_{1}(\zeta, \tau, \mu)$ of Appendix A.

In order to find the mean energy (or the partition function) we have to specify the space-time dimension $D$ together with the field dimension $d$. We will consider the following two basic examples.

\subsubsection{Canonical free massless field in even space time dimension $D$}

The canonical free field is determined by the Laplace equation

$$
\partial_{z}^{2} \phi^{(d)}(z)=0 \quad \Leftrightarrow \quad \partial_{z_{1}}^{2} \frac{1}{\left(z_{12}^{2}\right)^{d}}=0 \quad \Leftrightarrow \quad d=d_{0}:=\frac{D-2}{2} .
$$

The existence of the canonical free field as a GCI field requires $D$ to be even and greater than 2 . Then the polynomials $\widetilde{C}_{n}^{d_{0}}(z, w)$ are harmonic in both $z$ and $w$, and they determine a positive definite scalar product by Eq. (4.17). Thus, the canonical free fields satisfy the Hilbert space Wightman positivity. 
The operator-valued polynomials $\phi_{-n-d}(z)$ are harmonic, i. e.

$$
\phi_{-n-d}(z)=\sum_{\sigma} \phi_{\{0, n, \sigma\}} h_{\sigma}^{(n)}(z)
$$

in the notations of Sect. 2.2, so that the only nonzero modes of $\phi(z)$ are $\phi_{\{0, n, \sigma\}}$ and $\phi_{\left\{-n-d_{0}, n, \sigma\right\}}$ for $n=0,1, \ldots$ It then follows that the 1-particle eigenspace of conformal energy $n\left(\geqslant d_{0}\right)$ is isomorphic to the space of the harmonic polynomials on $\mathbb{C}^{D}$ of degree $n-d_{0}$. Its dimension $d^{(D)}(n)\left(=d(n) \equiv d_{B}(n)\right)$ is thus

$$
d^{(D)}(n)=\frac{2 n}{\left(2 d_{0}\right) !} \prod_{k=1-d_{0}}^{d_{0}-1}(n-k) \quad \text { for } \quad D>4 \quad\left(d^{(4)}(n)=n^{2}\right)
$$

which is an even polynomial in $n$, for even $D$, of degree $2 d_{0}$, say

$$
d^{(D)}(n)=\sum_{k=0}^{d_{0}} c_{k}^{(D)} n^{2 k}\left(=\frac{2 n^{2}}{\left(2 d_{0}\right) !} \prod_{k=1}^{d_{0}-1}\left(n^{2}-k^{2}\right) \quad \text { for } \quad D>4\right) .
$$

Note that $d^{(D)}(n)=0$ for $n=1, \ldots, d_{0}-1$ so that the thermal energy mean value is

$$
\begin{aligned}
\langle H\rangle_{q} & \equiv \frac{\operatorname{tr}_{\mathcal{D}}\left(H q^{H}\right)}{\operatorname{tr}_{\mathcal{D}}\left(q^{H}\right)}=\frac{1}{Z(\tau)} q \frac{\partial}{\partial q} Z(\tau)=\sum_{n=1}^{\infty} \frac{n d(n) q^{n}}{1-q^{n}}= \\
& =\sum_{k=1}^{d_{0}+1} c_{k-1}^{(D)} \frac{B_{2 k}}{4 k}+\sum_{k=1}^{d_{0}+1} c_{k-1}^{(D)} G_{2 k}(\tau)
\end{aligned}
$$

where $G_{2 k}(\tau)$ are the level 1 modular forms (A.10) (A.16) and $B_{2 k}$ are the Bernoulli numbers (see Appendix A). This agrees with Eq. (4.3) since here $d_{f}(n)=0$ and $d_{B}(n) \equiv d(n)$. Note that $d^{(2)}(n)=c_{0}^{(2)}=2$, while for $D \geq 4, c_{0}^{(D)}=0$. In particular, for $D=4$ we find

$$
\left\langle H+E_{0}\right\rangle_{q}^{(4)}=G_{4}(\tau), \quad E_{0}=\frac{1}{240} .
$$

If we interpret $E_{0}$ as a vacuum energy, i. e. renormalize the conformal Hamiltonian as $\widetilde{H}=H+E_{0}$ then its temperature mean value would be a modular form of weight 4 .

Remark 4.4. Extrapolation to the case $D=2$ of the above result contains two chiral components each of them giving the energy distribution for a $U(1)$ current

$$
\left\langle H+E_{0}\right\rangle_{q}^{(2)}=G_{2}(\tau), \quad E_{0}=-\frac{1}{24}
$$

which is not modular invariant. 


\subsubsection{Subcanonical field of dimension $d=1$ for $D=6$}

The scalar field of dimension $d=1$ in $D=6$ space-time dimensions is not harmonic but satisfies the fourth order equation $\left(\partial_{z}^{2}\right)^{2} \phi(z)=0$. The harmonic polynomials on $\mathbb{C}^{6}$ are now $\widetilde{C}_{n}^{2}(z, w)$. The identity

$$
C_{n}^{1}(t)=\frac{1}{n+1}\left(C_{n}^{2}(t)-C_{n-2}^{2}(t)\right)
$$

implies the following harmonic decomposition of the homogeneous polynomials $\widetilde{C}_{n}^{1}(z, w)$ :

$$
\widetilde{C}_{n}^{2}(z, w)=\frac{1}{n+1} \widetilde{C}_{n}^{2}(z, w)-\frac{1}{n+1} z^{2} w^{2} \widetilde{C}_{n-2}^{2}(z, w) .
$$

Thus we can decompose

$$
\phi_{-n-1}(z)=\phi_{-n-1}^{1}(z)+z^{2} \phi_{-n-1}^{2}(z),
$$

where $\phi_{n}^{j}(z)$ are now harmonic homogeneous operator-valued polynomials of degrees $n$ and $n-2$, respectively (as $\phi_{0}^{1}:=0$ and $\phi_{0}^{2}=\phi_{1}^{2}:=0$ ). Then,

$$
\begin{aligned}
& \left\langle 0\left|\phi_{-n-1}^{1 *}(z) \phi_{-n-1}^{1}(w)\right| 0\right\rangle=\frac{1}{n+1} \widetilde{C}_{n}^{2}(z, w) \\
& \left\langle 0\left|\phi_{-n-3}^{2 *}(z) \phi_{-n-3}^{2}(w)\right| 0\right\rangle=\frac{-1}{n+1} \widetilde{C}_{n}^{2}(z, w) .
\end{aligned}
$$

Therefore, the 1-particle state space of conformal energy $n$ decomposes into a pseudo-orthogonal direct sum of two subspaces isomorphic to the spaces of harmonic homogeneous polynomials of degrees $n-1$ and $n-3$, respectively: the first will have positive definite while the second one, negative definite metric. In particular, the dimension of the full eigenspace of conformal energy $n$ is

$$
d_{B}(n)=d^{(6)}(n+1)+d^{(6)}(n-1)=\frac{n^{2}\left(n^{2}+5\right)}{6}
$$

so that the thermal energy mean value and the vacuum energy are

$$
\left\langle H+E_{0}\right\rangle_{q}=\frac{1}{6} G_{6}(\tau)+\frac{5}{6} G_{4}(\tau), \quad E_{0}=-\frac{1}{6} \frac{B_{6}}{12}-\frac{5}{6} \frac{B_{4}}{8}=\frac{19}{6048} .
$$

\subsection{The Weyl field}

Let us introduce the $(2 \times 2)$-matrix representation of the quaternionic algebra:

$$
\begin{gathered}
Q_{k}=-i \sigma_{k}=-Q_{k}^{+}(k=1,2,3), \quad Q_{4}=\mathbb{I} \\
\sigma_{1}=\left(\begin{array}{ll}
0 & 1 \\
1 & 0
\end{array}\right), \quad \sigma_{2}=\left(\begin{array}{cc}
0 & -i \\
i & 0
\end{array}\right), \quad \sigma_{3}=\left(\begin{array}{cc}
1 & 0 \\
0 & -1
\end{array}\right), \\
Q_{\alpha}^{+} Q_{\beta}+Q_{\beta}^{+} Q_{\alpha}=2 \delta_{\alpha \beta}=Q_{\alpha} Q_{\beta}^{+}+Q_{\beta} Q_{\alpha}^{+} \quad \text { for } \quad \alpha, \beta=1, \ldots, 4
\end{gathered}
$$

( $\sigma_{k}$ being Pauli matrices). In this section we will denote the hermitian matrix conjugation by a superscript "+". The matrices

$$
i \sigma_{\alpha \beta}=\frac{1}{2}\left(Q_{\alpha}^{+} Q_{\beta}-Q_{\beta}^{+} Q_{\alpha}\right), \quad i \widetilde{\sigma}_{\alpha \beta}=\frac{1}{2}\left(Q_{\alpha} Q_{\beta}^{+}-Q_{\beta} Q_{\alpha}^{+}\right)
$$


are the selfdual and antiselfdual antihermitian spin (4) Lie algebra generators. We will denote also

$$
\not=\sum_{\alpha=1}^{4} z^{\alpha} Q_{\alpha}, \quad \not^{+}=\sum_{\alpha=1}^{4} z^{\alpha} Q_{\alpha}^{+}, \quad \not_{z}=\sum_{\alpha=1}^{4} Q_{\alpha} \partial_{z^{\alpha}}, \quad \phi_{z}^{+}=\sum_{\alpha=1}^{4} Q_{\alpha}^{+} \partial_{z^{\alpha}},
$$

etc. Note that in the definition of $\not^{+}$we do not conjugate the coordinates $z^{\alpha}$. Then Eqs. (4.42) are equivalent to

$$
\not_{1}^{+} \not k_{2}+\not_{2}^{+} \not_{1}=\not_{1} k_{2}^{+}+k_{2} k_{1}^{+}=2 z_{1} \cdot z_{2} \quad\left(\not^{+} \not k=\not k^{+}=z^{2}\right) \text {. }
$$

The generalized free Weyl fields of dimension $d=\frac{1}{2}, \frac{3}{2}, \ldots$ are two mutually conjugate complex 2-component fields,

$$
\chi^{+}(z)=\left(\stackrel{*}{\chi_{1}}(z), \stackrel{*}{\chi_{2}}(z)\right) \text { and } \chi(z)=\left(\begin{array}{c}
\chi_{1}(z) \\
\chi_{2}(z)
\end{array}\right)
$$

transforming under the elementary induced representations of spin (4) corresponding to the selfdual and antiselfdual representations (4.43), respectively. In particular, the action of the Weyl reflection $j_{W}(2.7)$ is,

$$
\begin{aligned}
& \chi(z) \longmapsto \frac{\not}{\left(z^{2}\right)^{d+\frac{1}{2}}} \chi(z)\left(\equiv \pi_{z}\left(j_{W}\right) \chi(z)\right), \\
& \chi^{+}(z) \longmapsto \chi^{+}(z) \frac{\not}{\left(z^{2}\right)^{d+\frac{1}{2}}}\left(\equiv \pi_{z}^{+}\left(j_{W}\right) \chi^{+}(z)\right) .
\end{aligned}
$$

The conformal invariant 2-point functions, characterizing the fields, have the following matrix representation

$$
\begin{gathered}
\left\langle 0\left|\chi\left(z_{1}\right) \chi^{+}\left(z_{2}\right)\right| 0\right\rangle=\frac{\not_{12}^{+}}{\left(z_{12}^{2}\right)^{d+\frac{1}{2}}}, \\
\left\langle 0\left|\chi_{\alpha}\left(z_{1}\right) \chi_{\beta}\left(z_{2}\right)\right| 0\right\rangle=\left\langle 0\left|\chi_{\alpha}^{+}\left(z_{1}\right) \chi_{\beta}^{+}\left(z_{2}\right)\right| 0\right\rangle=0 .
\end{gathered}
$$

In particular, the invariance under the complex Weyl reflection $j_{W}$ is ensured by the equality

$$
\frac{\not k_{1}}{z_{1}^{2}} \not_{12}^{+} \frac{\star_{2}}{z_{2}^{2}}=\frac{\not_{1}^{+}}{z_{1}^{2}}-\frac{\not_{2}^{+}}{z_{2}^{2}} \text {. }
$$

The conjugation law (2.21) reads

$$
\chi^{+}(\bar{z})^{+}=\frac{\not}{\left(z^{2}\right)^{d+\frac{1}{2}}} \chi\left(\frac{z}{z^{2}}\right)
$$

in other words, for any $\Phi, \Psi \in \mathcal{D}$ :

$$
\left\langle\chi^{+}(\bar{z}) \Phi \mid \Psi\right\rangle=\frac{\not}{\left(z^{2}\right)^{d+\frac{1}{2}}}\left\langle\Phi \mid \chi\left(\frac{z}{z^{2}}\right) \Psi\right\rangle \text {. }
$$


Here one can explicitly verify the hermiticity of the 2-point scalar product

$$
\left\langle\chi^{+}(\bar{z}) \Omega \mid \chi^{+}(w) \Omega\right\rangle=\left(\left\langle\chi^{+}(w) \Omega \mid \chi^{+}(\bar{z}) \Omega\right\rangle\right)^{+},
$$

where $\Omega=|0\rangle$ is the vacuum:

$$
\frac{\not k}{\left(z^{2}\right)^{d+\frac{1}{2}}}\left\langle 0\left|\chi\left(\frac{z}{z^{2}}\right) \chi^{+}(w)\right| 0\right\rangle=\left(\frac{\bar{\psi}}{\left(\bar{w}^{2}\right)^{d+\frac{1}{2}}}\left\langle 0\left|\chi\left(\frac{\bar{w}}{\bar{w}^{2}}\right) \chi^{+}(\bar{z})\right| 0\right\rangle\right)^{+} .
$$

The conjugation law for the compact picture generalized free Weyl field

$$
\chi^{+}(\zeta, u)=e^{2 \pi i d \zeta} \chi^{+}\left(e^{2 \pi i \zeta} u\right), \quad \chi(\zeta, u)=e^{2 \pi i d \zeta} \chi\left(e^{2 \pi i \zeta} u\right)
$$

becomes

$$
\chi^{+}(\zeta, u)^{+}=\psi \chi(\zeta, u) .
$$

The vacuum correlation function is diagonal in "the moving frame" representation defined as follows. For given non-collinear unit real vectors $u_{1}, u_{2} \in \mathbb{S}^{D-1}(\subset$ $\mathbb{R}^{D}$ ) such that $u_{1} \cdot u_{2}=\cos 2 \pi \alpha$ let $v$ and $\bar{v}$ be the unique complex vectors (in $\mathbb{C}^{D}$ ) for which

$$
u_{1}=e^{\pi i \alpha} v+e^{-\pi i \alpha} \bar{v}, \quad u_{2}=e^{-\pi i \alpha} v+e^{\pi i \alpha} \bar{v} .
$$

It then follows that $v$ and $\bar{v}$ are mutually conjugate isotropic vectors with scalar product: $2 v \cdot \bar{v}=1$. In this basis we have

$$
\left\langle 0\left|\chi\left(\zeta_{1}, u_{1}\right) \chi^{+}\left(\zeta_{2}, u_{2}\right)\right| 0\right\rangle=\frac{1}{2 i\left(-4 \sin \pi \zeta_{+} \sin \pi \zeta_{-}\right)^{d-\frac{1}{2}}}\left(\frac{\psi^{+}}{\sin \pi \zeta_{-}}+\frac{\bar{\psi}^{+}}{\sin \pi \zeta_{+}}\right),
$$

where $\zeta_{ \pm}=\zeta_{12} \pm \alpha$ (as in previous sections). In the frame, in which $u_{1,2}=(0,0$, $\pm \sin \pi \alpha, \cos \pi \alpha)$ the matrix $\psi$ and its conjugate assume a simple form:

$$
\psi^{+}=\left(\begin{array}{ll}
1 & 0 \\
0 & 0
\end{array}\right)=\bar{\psi}, \quad \bar{\psi}^{+}=\left(\begin{array}{ll}
0 & 0 \\
0 & 1
\end{array}\right)=\psi .
$$

Thus, in the $d=\frac{1}{2}$ case of a subcanonical Weyl field the contribution of $\zeta_{+}$and $\zeta_{-}$ are separated. The dimension $d=\frac{3}{2}$ corresponds to the canonical free Weyl field which will be denoted by $\psi:=\chi\left(\psi^{+}:=\chi^{+}\right)$. We find in this case

$$
\begin{gathered}
\left\langle 0\left|\psi\left(\zeta_{1}, u_{1}\right) \psi^{+}\left(\zeta_{2}, u_{2}\right)\right| 0\right\rangle=\frac{i}{8 \sin 2 \pi \alpha}\left(\psi ^ { + } \left(\frac{\cos \pi \zeta_{-}}{\sin ^{2} \pi \zeta_{-}}-\frac{\cot 2 \pi \alpha}{\sin \pi \zeta_{-}}+\right.\right. \\
\left.\left.\quad+\frac{1}{\sin 2 \pi \alpha \sin \pi \zeta_{+}}\right)-\bar{\psi}^{+}\left(\frac{\cos \pi \zeta_{+}}{\sin ^{2} \pi \zeta_{+}}+\frac{\cot 2 \pi \alpha}{\sin \pi \zeta_{+}}-\frac{1}{\sin 2 \pi \alpha \sin \pi \zeta_{-}}\right)\right),
\end{gathered}
$$

From the vacuum correlation functions (4.58), (4.60) and from Eq. (4.6) we deduce

$$
\begin{aligned}
& \left\langle\chi\left(\zeta_{1}, u_{1}\right) \chi^{+}\left(\zeta_{2}, u_{2}\right)\right\rangle_{q}=\frac{1}{2 \pi i}\left(p_{1}^{11}\left(\zeta_{-}, \tau\right) \psi^{+}+p_{1}^{11}\left(\zeta_{+}, \tau\right) \bar{\psi}^{+}\right) \\
& \left\langle\psi\left(\zeta_{1}, u_{1}\right) \psi^{+}\left(\zeta_{2}, u_{2}\right)\right\rangle_{q}=\frac{i}{8 \pi \sin 2 \pi \alpha}\left(\psi ^ { + } \left(p_{2}^{11}\left(\zeta_{-}, \tau\right)-\cot 2 \pi \alpha p_{1}^{11}\left(\zeta_{-}, \tau\right)+\right.\right. \\
& \left.\left.\quad+\frac{p_{1}^{11}\left(\zeta_{+}, \tau\right)}{\sin 2 \pi \alpha}\right)-\bar{\psi}^{+}\left(p_{2}^{11}\left(\zeta_{+}, \tau\right)+\cot 2 \pi \alpha p_{1}^{11}\left(\zeta_{+}, \tau\right)-\frac{p_{1}^{11}\left(\zeta_{-}, \tau\right)}{\sin 2 \pi \alpha}\right)\right)
\end{aligned}
$$


Here $p_{k}^{\kappa, \lambda}(\zeta, \tau)=\sum_{m} \sum_{n}(-1)^{m \kappa+n \lambda}(\zeta+m \tau+n)^{-k}$ (see Appedix A). Under the assumptions of Remark 4.1, for $N$ identified with the charge operator (so that $\left.\left[N, \chi^{+}(z)\right]=\chi^{+}(z),[N, \chi(z)]=-\chi(z)\right)$, we find for $d=\frac{1}{2}$

$$
\left\langle\chi\left(\zeta_{1}, u_{1}\right) \chi^{+}\left(\zeta_{2}, u_{2}\right)\right\rangle_{q, \mu}=\frac{1}{2 \pi i}\left(p_{1}^{11}\left(\zeta_{-}, \tau, \mu\right) \not^{+}+p_{1}^{11}\left(\zeta_{+}, \tau, \mu\right) \bar{\psi}^{+}\right)
$$

using the more general functions $p_{1}^{\kappa, \lambda}(\zeta, \tau, \mu)$ of Appendix A (Eq. (4.62) is generalized in a similar way).

Note that the 1-particle scalar product is

$$
\left\langle 0\left|\chi^{+}(\bar{z})^{+} \chi^{+}(w)\right| 0\right\rangle=\frac{1-\not \psi^{+}}{\left(1-2 z \cdot w+z^{2} w^{2}\right)^{d+\frac{1}{2}}} .
$$

This implies similarly to the scalar field case that we can organize the field mode expansion (in the compact picture) as

$$
\chi(\zeta, u)=\sum_{\substack{n \in \mathbb{Z} \\\left|n-\frac{1}{2}\right| \geqslant d}} \chi_{n-\frac{1}{2}}(u) e^{i \pi(1-2 n) \zeta}, \chi^{+}(\zeta, u)=\sum_{\substack{n \in \mathbb{Z} \\\left|n-\frac{1}{2}\right| \geqslant d}} \chi_{n-\frac{1}{2}}^{+}(u) e^{i \pi(1-2 n) \zeta},
$$

where $\chi_{n+d}^{(+)}(u)$ and $\chi_{-n-d}^{(+)}(u)$ for $n=0,1, \ldots$ are homogeneous polynomial in $u \in \mathbb{S}^{3}$ of degree $n$ with 2 -component operator coefficients. For $n \geqslant 0, \chi_{n+d}^{(+)}(u)$ correspond to annihilation operators while $\chi_{-n-d}^{(+)}(u)$, to the creation modes and we also have

$$
\begin{gathered}
\left(\chi_{\frac{1}{2}-k}^{+}(u)\right)^{+}=\psi \chi_{k-\frac{1}{2}}(u), \quad\left\langle 0\left|\chi_{-m-d}^{+}\left(u_{1}\right)^{+} \chi_{-n-d}^{+}\left(u_{2}\right)\right| 0\right\rangle= \\
=\delta_{n m}\left(C_{n}^{d+\frac{1}{2}}\left(u_{1} \cdot u_{2}\right)-C_{n-1}^{d+\frac{1}{2}}\left(u_{1} \cdot u_{2}\right) \psi_{1} \psi_{2}^{+}\right)
\end{gathered}
$$

for $k \in \mathbb{Z}, n, m=0,1, \ldots$ and $u_{1}, u_{2} \in \mathbb{S}^{3}$.

For the thermal energy mean values we will consider the two cases of $d=\frac{3}{2}$ and $d=\frac{1}{2}$, separately.

The $z$-picture canonical spinor field satisfies the Weyl-Dirac equation

$$
\not_{z} \psi(z)=0, \quad \psi^{+}(z) \overleftarrow{\not}_{z}=0
$$

These equations are also valid for the compact picture modes $\psi_{-n-\frac{3}{2}}(u)$ extended to $u \in \mathbb{R}^{4}$. The positive charge 1-particle state-space of conformal energy $\langle 0|H| 0\rangle+$ $n+\frac{3}{2}(n=0,1, \ldots)$, spanned by $\psi_{-n-\frac{3}{2}}^{+}(u)|0\rangle$ carries the irreducible representation $\left(\frac{n}{2}, \frac{n+1}{2}\right)$ of $\operatorname{Spin}(4)$ and therefore, has dimension $(n+2)(n+1)$. The dimension of the full 1-particle space, including charge -1 states, is twice as big. It has also 
a positive definite scalar product in view of [24]. Thus applying the general formula (4.3) and Eq. (A.10) we find (cf. [10]):

$$
\begin{gathered}
\left\langle H+E_{0}\right\rangle_{q}=E_{0}+\frac{1}{Z(\tau)} q \frac{\partial}{\partial q} Z(\tau)=\sum_{n=0}^{\infty} \frac{2\left(n+\frac{3}{2}\right)(n+1)(n+2) q^{n+\frac{3}{2}}}{1+q^{n+\frac{3}{2}}}= \\
=\frac{1}{4}\left(G_{4}\left(\frac{\tau+1}{2}\right)-8 G_{4}(\tau)\right)-\frac{1}{4}\left(G_{2}\left(\frac{\tau+1}{2}\right)-2 G_{2}(\tau)\right), \\
E_{0}=-\frac{1}{4} \frac{B_{4}}{8}\left(1-2^{3}\right)+\frac{1}{4} \frac{B_{2}}{4}(1-2)=-\frac{17}{960} .
\end{gathered}
$$

Here we have used the equalities

$$
\begin{gathered}
\sum_{n=1}^{\infty} \frac{(2 n+1)^{2 k-1} q^{2 n+1}}{1+q^{2 n+1}}=G_{2 k}\left(\tau+\frac{1}{2}\right)-2^{2 k-1} G_{2 k}(2 \tau)+\frac{B_{2 k}}{4 k}\left(1-2^{2 k-1}\right), \\
2\left(n+\frac{1}{2}\right) n(n+1)=\frac{1}{4}\left((2 n+1)^{3}-(2 n+1)\right) .
\end{gathered}
$$

The subcanonical Weyl field and its conjugate satisfy third order equations which assume the following form on the modes

$$
\partial_{u}^{2} \not_{u} \chi_{n+\frac{1}{2}}(u)=0, \quad \chi_{n+\frac{1}{2}}^{+}(u) \overleftarrow{\not}_{u} \overleftarrow{\partial}_{u}^{2}=0 \quad\left(u \in \mathbb{R}^{3}\right)
$$

The resulting Spin (4)-representation in the positive charge 1-particle space of conformal energy $n+\frac{1}{2}(n=0,1, \ldots)$ is then isomorphic to a (pseudoorthogonal) direct sum of three irreducible representations (for $n \geqslant 3$ ),

$$
\left(\frac{n}{2}, \frac{n+1}{2}\right) \oplus\left(\frac{n}{2}, \frac{n-1}{2}\right) \oplus\left(\frac{n-2}{2}, \frac{n-1}{2}\right)
$$

each of them should posses a definite restriction of the scalar product. In particular, the full dimension $d_{f}(n)$ of the space (4.73) is

$$
\frac{1}{2} d_{f}(n)=3 n^{2}+3 n+2, \quad\left(n+\frac{1}{2}\right) d_{f}(n)=\frac{3}{4}(2 n+1)^{3}+\frac{5}{4}(2 n+1),
$$

for all $n=0,1, \ldots$, so that the thermal energy mean value and vacuum energy are given by

$$
\begin{gathered}
\left\langle H+E_{0}\right\rangle_{q}=\frac{3}{4}\left(G_{4}\left(\frac{\tau+1}{2}\right)-8 G_{4}(\tau)\right)+\frac{5}{4}\left(G_{2}\left(\frac{\tau+1}{2}\right)-2 G_{2}(\tau)\right), \\
E_{0}=-\frac{3}{4} \frac{B_{4}}{8}\left(1-2^{3}\right)-\frac{5}{4} \frac{B_{2}}{4}(1-2)=\frac{29}{960} .
\end{gathered}
$$

Note that although $G_{2}$ is not a modular form the differences entering the right hand sides of (4.65) and (4.75) are multiples of $F(\tau)($ A.15) and are thus modular forms of weight two and level $\Gamma_{\theta}$. 


\subsection{The Maxwell free field}

The electromagnetic (or Maxwell) free field is a 6 component field $F_{\alpha \beta}(z)=$ $-F_{\beta \alpha}(z)(1 \leqslant \alpha<\beta \leqslant 4)$. It is convenient to write it as a 2 -form:

$$
F(z)=\frac{1}{2} F_{\alpha \beta}(z) d z^{\alpha} \wedge d z^{\beta}
$$

which makes clear its transformation properties and conjugation law:

$$
\begin{gathered}
U(g)\left(F_{\alpha \beta}(z) d z^{\alpha} \wedge d z^{\beta}\right) U\left(g^{-1}\right)=F_{\alpha \beta}(g(z)) d g(z)^{\alpha} \wedge d g(z)^{\beta}, \\
\left(F_{\alpha \beta}(z) d z^{\alpha} \wedge d z^{\beta}\right)^{*}=F_{\alpha \beta}\left(z^{*}\right) d \bar{z}^{\alpha} \wedge d \bar{z}^{\beta} \quad\left(z^{*}=\frac{\bar{z}}{\bar{z}^{2}}, \quad\left(d z^{\alpha}\right)^{*}=d\left(z^{*}\right)^{\alpha}\right),
\end{gathered}
$$

where $g=e^{t \Omega}$ for a real conformal generator $\Omega$ as in Eq. (2.15). The 2-point function is

$$
\left\langle 0\left|F_{\alpha_{1} \beta_{1}}\left(z_{1}\right) F_{\alpha_{2} \beta_{2}}\left(z_{2}\right)\right| 0\right\rangle:=\frac{r_{\alpha_{1} \alpha_{2}}\left(z_{12}\right) r_{\beta_{1} \beta_{2}}\left(z_{12}\right)-r_{\alpha_{1} \beta_{2}}\left(z_{12}\right) r_{\beta_{1} \alpha_{2}}\left(z_{12}\right)}{\left(z_{12}^{2}\right)^{2}},
$$

$r_{\alpha \beta}(z):=\delta_{\alpha \beta}-2 \frac{z_{\alpha} z_{\beta}}{z^{2}}$. It is verified to satisfy the Maxwell equations

$$
d F(z)=0, \quad d *(F)(z)=0,
$$

* being Hodge star: $*(F)_{\alpha \beta}(z):=\varepsilon_{\alpha \beta \rho \sigma} F^{\rho \sigma}(z)$.

To compute the (compact picture) finite temperature correlation functions $\left\langle F_{\alpha_{1} \beta_{1}}\left(\zeta_{1}, u_{1}\right) F_{\alpha_{2} \beta_{2}}\left(\zeta_{2}, u_{2}\right)\right\rangle_{q}$ we again use the diagonal frame in which, $2 v=$ $(0,0,-i, 1), u_{1,2}=(0,0, \pm \sin \pi \alpha, \cos \pi \alpha)$; then there exist linear combinations of the field components

$$
\sqrt{2} F_{1}^{ \pm}=F_{23} \pm F_{14}, \sqrt{2} F_{2}^{ \pm}=F_{31} \pm F_{24}, \sqrt{2} F_{3}^{ \pm}=F_{12} \pm F_{34}, \sqrt{2} F_{ \pm}^{\varepsilon}=F_{1}^{\varepsilon} \pm i F_{2}^{\varepsilon}
$$

$(\varepsilon= \pm)$ such that

$$
\begin{gathered}
\left\langle 0\left|F_{+}^{+}\left(\zeta_{1}, u_{1}\right) F_{-}^{-}\left(\zeta_{2}, u_{2}\right)\right| 0\right\rangle=: \mathcal{W}_{0}\left(\zeta_{12}, \alpha\right)= \\
=\frac{1}{4 \sin ^{3} 2 \pi \alpha}\left(\operatorname{cotg} \pi \zeta_{-}-\operatorname{cotg} \pi \zeta_{+}\right)-\frac{1}{4 \sin 2 \pi \alpha}\left(\frac{\cos \pi \zeta_{+}}{\sin ^{3} \pi \zeta_{+}}-\frac{\operatorname{cotg} 2 \pi \alpha}{\sin ^{2} \pi \zeta_{+}}\right) ; \\
\left\langle 0\left|F_{-}^{+}\left(\zeta_{1}, u_{1}\right) F_{+}^{-}\left(\zeta_{2}, u_{2}\right)\right| 0\right\rangle=\mathcal{W}_{0}\left(\zeta_{12},-\alpha\right) ; \quad\left\langle 0\left|F_{3}^{+}\left(\zeta_{1}, u_{1}\right) F_{3}^{-}\left(\zeta_{2}, u_{2}\right)\right| 0\right\rangle= \\
=\frac{1}{4 \sin ^{2} 2 \pi \alpha}\left(\frac{1}{\sin ^{2} \pi \zeta_{+}}+\frac{1}{\sin ^{2} \pi \zeta_{-}}+2 \operatorname{cotg} 2 \pi \alpha\left(\operatorname{cotg} \pi \zeta_{+}-\operatorname{cotg} \pi \zeta_{-}\right)\right)
\end{gathered}
$$

$\left(\zeta_{ \pm}=\zeta_{12} \pm \alpha\right)$. The corresponding finite temperature correlation functions read:

$$
\begin{gathered}
\left\langle F_{+}^{+}\left(\zeta_{1}, u_{1}\right) F_{-}^{-}\left(\zeta_{2}, u_{2}\right)\right\rangle_{q}=: \mathcal{W}_{q}\left(\zeta_{12}, \alpha\right)=\frac{1}{4 \sin ^{3} 2 \pi \alpha}\left(p_{1}\left(\zeta_{-}, \tau\right)-p_{1}\left(\zeta_{+}, \tau\right)\right)- \\
-\frac{1}{4 \sin 2 \pi \alpha}\left(\frac{1}{2 \pi} p_{3}\left(\zeta_{+}, \tau\right)-\operatorname{cotg} 2 \pi \alpha p_{2}\left(\zeta_{+}, \tau\right)\right) ; \\
\left\langle F_{-}^{+}\left(\zeta_{1}, u_{1}\right) F_{+}^{-}\left(\zeta_{2}, u_{2}\right)\right\rangle_{q}=\mathcal{W}_{q}\left(\zeta_{12},-\alpha\right) ; \quad\left\langle F_{3}^{+}\left(\zeta_{1}, u_{1}\right) F_{3}^{-}\left(\zeta_{2}, u_{2}\right)\right\rangle_{q}= \\
=\frac{1}{4 \sin ^{2} 2 \pi \alpha}\left(p_{2}\left(\zeta_{+}, \tau\right)+p_{2}\left(\zeta_{-}, \tau\right)+2 \operatorname{cotg} 2 \pi \alpha\left(p_{1}\left(\zeta_{+}, \tau\right)-p_{1}\left(\zeta_{-}, \tau\right)\right)\right) .
\end{gathered}
$$


In order to find the thermal energy mean value for the Maxwell field we have to compute the dimension $d(n)\left(\equiv d_{B}(n)\right)$ of the 1-particle state space of conformal energy $n$, spanned by $F_{\alpha \beta ;-n}(z)|0\rangle$ where the mode $F_{\alpha \beta ;-n}(z)$ is a homogeneous (harmonic) polynomial of degree $n-2$, satisfying the Maxwell equations. To this end we display the $S O(4)$ representation content of the modes satisfying the Maxwell equations. Decomposing the antisymmetric tensor $F_{\alpha \beta}$ into selfdual and antiselfdual parts, $(1,0) \oplus(0,1)$, we see that the full space of homogeneous skewsymmetric-tensor valued polynomials in $z$ of degree $n-2$ generically splits into a direct sum of three conjugate pairs of $S U(2) \times S U(2)$ representations; for instance, $(1,0) \otimes\left(\frac{n-2}{2}, \frac{n-2}{2}\right)=\left(\frac{n}{2}, \frac{n-2}{2}\right) \oplus\left(\frac{n-2}{2}, \frac{n-2}{2}\right) \oplus\left(\frac{n-4}{2}, \frac{n-2}{2}\right)$ (for $n>3$ ). Maxwell equations imply that only two of the resulting six representations, those with maximal weights, appear in the energy $n$ 1-particle space: $\left(\frac{n}{2}, \frac{n-2}{2}\right) \oplus\left(\frac{n-2}{2}, \frac{n}{2}\right)$. Thus,

$$
d(n)=2\left(n^{2}-1\right)
$$

and using (4.32) we then find

$$
\left\langle H+E_{0}\right\rangle_{q}=2 G_{4}(\tau)-2 G_{2}(\tau), \quad E_{0}=-2 \frac{B_{4}}{8}+2 \frac{B_{2}}{4}=\frac{11}{120} .
$$

Remark 4.5. Let us consider a generalized free vector field $l_{\alpha}(z)$ independent of $F_{\alpha \beta}(z)$ (i. e., commuting with it) and having two point function

$$
\left\langle 0\left|\iota_{\alpha}\left(z_{1}\right) \iota_{\beta}\left(z_{2}\right)\right| 0\right\rangle=C \frac{r_{\alpha \beta}\left(z_{12}\right)}{z_{12}^{2}}, \quad \partial_{z^{\alpha}} l_{\beta}(z)=\partial_{z^{\beta}} l_{\alpha}(z)
$$

(the last equality means that $\kappa_{\alpha}(z)$ is a "longitudinal" field but we note that there is no GCI scalar field $s(z)$ such that $\left.l_{\alpha}(z)=\partial_{z^{\alpha}} s(z)\right)$. The field $l_{\alpha}(z)$ satisfies the third order equation

$$
\partial_{z}^{2} \partial_{z} \cdot \mathcal{l}(z)=0
$$

and it then follows that the conformal energy $n$ state space has dimension

$$
d_{l}(n)=\left(\begin{array}{c}
n+3 \\
3
\end{array}\right)-\left(\begin{array}{c}
n-1 \\
3
\end{array}\right)=(n+1)^{2}+(n-1)^{2}=2\left(n^{2}+1\right) .
$$

Thus the thermal energy mean value in the state space of $F_{\alpha \beta}(z)$ and $l_{\alpha}(z)$ will be

$$
\left\langle H_{F}+H_{l}+E_{0}\right\rangle_{q}=4 G_{4}(\tau), \quad E_{0}=\frac{1}{60}
$$

( $H_{F}$ and $H_{l}$ being the conformal Hamiltonians of the corresponding subsystems). We can interpret the state space of $\tau_{\alpha}(z)$ as the space of pure gauge transformations and then the full state space of $F_{\alpha \beta}(z)$ and $l_{\alpha}(z)$ has the meaning of the space of all gauge potentials. 


\section{The Thermodynamic Limit}

\subsection{Compactified Minkowski space as a "finite box" approximation}

We shall now substitute $z$ in Eqs. (1.3) and (2.3) by $\frac{z}{R}$ thus treating $\mathbb{S}^{D-1}$ and $\mathbb{S}^{1}$ in the definition of $\bar{M}$ as a sphere and a circle of radius $R(>0)$. Performing further the Minkowski space dilation $(2 R)^{\Omega_{-1 D}}: x^{\mu} \mapsto \frac{x^{\mu}}{2 R}, \mu=0, \ldots, D-1$ (see Eq. (2.2)) on the (real) variable $(\mathcal{z}=) x$ in $(2.3)$ we find $z(x ; R)=R z\left(\frac{x}{2 R}\right)$ or

$$
\boldsymbol{z}(x ; R)=\frac{\boldsymbol{x}}{2 \omega\left(\frac{x}{2 R}\right)}, z_{D}(x ; R)-R=\frac{i x^{0}-\frac{x^{2}}{2 R}}{2 \omega\left(\frac{x}{2 R}\right)}, 2 \omega\left(\frac{x}{2 R}\right)=1+\frac{x^{2}}{4 R^{2}}-i \frac{x^{0}}{R} .
$$

The stability subgroup of $z(x ; R)=0\left(\in T_{+}\right)$in $\mathcal{C}$ is conjugate to the maximal compact subgroup $\mathcal{K} \subset \mathcal{C}$ :

$$
\mathcal{K}(2 R)=(2 R)^{\Omega_{-1 D}} \mathcal{K}(2 R)^{-\Omega_{-1 D}}, \quad \mathcal{K} \equiv \mathcal{K}(1) \cong U(1) \times \operatorname{Spin}(D) / \mathbb{Z}_{2} .
$$

In particular, the hermitian $U(1)$-generator $H(2 R)$, which acts in the $z-$ coordinates (5.1) as the Euler vector field $z \cdot \frac{\partial}{\partial z}$, is mutually conjugate to $H \equiv H(1)$,

$$
H(2 R)=(2 R)^{\Omega_{-1 D}} H(2 R)^{-\Omega_{-1 D}}, \quad H \equiv H(1) .
$$

For large $R$ and finite $x$ the variables $\left(\boldsymbol{z}, z_{D}-R\right)$ approach the (Wick rotated) Minkowski space coordinates $\left(\boldsymbol{x}, i x^{0}\right)$. In particular, for $x^{0}=0(=\zeta)$, the real $(D-1)$-sphere $z^{2}=R^{2}$ can be viewed as a $S O(D)$-invariant "box" approaching for $R \rightarrow \infty$ the flat space $\mathbb{R}^{D-1}$. Thus the conformal compactification of Minkowski space also plays the role of a convenient tool for studying the thermodynamic limit of thermal expectation values. This interpretation is justified in view of the following:

Proposition 5.1. The asymptotic behaviour of $z(x ; R)-\operatorname{Re}_{D}\left(e_{D}=(\mathbf{0}, 1)\right)$ for large $R$ is:

$$
\begin{gathered}
z(x ; R)=\boldsymbol{x}+O\left(\frac{\|x\|^{2}}{R}\right), \quad z_{D}(x ; R)-R=i x^{0}+O\left(\frac{\|x\|^{2}}{R}\right), \\
H_{R}:=\frac{H(2 R)}{R}=P_{0}+\frac{1}{4 R^{2}} K_{0}\left(=P_{0}+O\left(\frac{1}{R}\right) \in i c\right),
\end{gathered}
$$

where $\|x\|:=\sqrt{\left(x^{0}\right)^{2}+|\boldsymbol{x}|^{2}}$ for $x=\left(x^{0}, \boldsymbol{x}\right) \in M$ and $i P_{0}$ is the real conformal algebra generator of the Minkowski time $\left(x^{0}\right)$ translation (see Sect. 2.1). The operator $H_{R}$ is the physical conformal Hamiltonian (of dimension inverse length). 
Proof. Eq. (5.4) is obtained by a straightforward computation. It follows from Eqs. (2.2) and (2.9) that

$$
H=\frac{1}{2}\left(P_{0}+K_{0}\right) .
$$

To derive Eq. (5.5) one should then use (5.6) and the equations $\lambda^{\Omega_{-1 D}} P_{0} \lambda^{-\Omega_{-1 D}}=$ $\lambda P_{0}, \lambda^{\Omega_{-1 D}} K_{0} \lambda^{-\Omega_{-1 D}}=\lambda^{-1} K_{0}$; hence, $H(2 R)=(2 R)^{\Omega_{-1 D}} H(2 R)^{-\Omega_{-1 D}}=R P_{0}+$ $\frac{1}{4 R} K_{0}$.

Remark 5.1. The observation that the universal cover of $\bar{M}$, the Einstein universe $\widetilde{M}=\mathbb{R} \times \mathbb{S}^{D-1}$ (for $D=4$ ), which admits a globally causal structure, is locally undistiguishable from $M$ for large $R$ has been emphasized over 30 year ago by Irving Segal (for a concise exposé and further references - see [32]). For a fixed choice, $\Omega_{-1 D}$, of the dilation generator in (5.2) he identifies the Minkowski energy $P_{0}$ with the scale covariant component of $H_{R}$. With this choice $M$ is osculating $\bar{M}$ (and hence $\widetilde{M}$ ) at the north pole $\left(\boldsymbol{z}, z_{D}\right)=(\mathbf{0}, R)$ (respectively, $\zeta=0, u=e_{D}$ ), identified with the origin $x=0$ in $M$. (The vector fields associated with $H_{R}$ and $P_{0}$ coincide at this point.)

Using the Lie algebra limit $\lim _{R \rightarrow \infty} H_{R}=P_{0}$ implied by (5.5), one can approximate the Minkowski energy operator $P_{0}$ for large $R$ by the physical conformal Hamiltonian $H_{R}$. As we shall see below, the fact that in all considered free field models in dimension $D=4$ the conformal mean energy is a linear combination of modular forms $G_{2 k}(\tau)$ with highest weight $2 k=4$, has a remarkable corollary: the density $\mathcal{E}$ of the physical mean energy has a limit reproducing the Stefan-Boltzmann law

$$
\mathcal{E}(\beta):=\lim _{R \rightarrow \infty} \frac{\left\langle H_{R}\right\rangle_{q_{\beta}}}{\mathcal{V}_{R}}=\frac{C}{\beta^{4}} \quad \text { for } \quad q_{\beta}:=e^{-\beta}
$$

where $C$ is some constant, $\beta=\frac{1}{k T}$ is the inverse temperature $T$ multiplied by the Boltzmann constant $k$ ) and $\mathcal{V}_{R}:=2 \pi^{2} R^{3}$ is the volume of the 3 -sphere of radius $R$ at a fixed time (say $x^{0}=0=\zeta$ ). We will calculate this limit for two cases: the model of a free scalar filed in $D=4$ (see Sect. 4.2.1) which we will further denote by $\varphi$ and the Maxwell free field model introduced in Sect. 4.4.

Proposition 5.2. For the free scalar field $\varphi$ in dimension $D=4$ we have the following behaviour of the mean energy density for $\frac{R}{\beta} \gg 1$

$$
\mathcal{E}_{R}^{(\varphi)}(\beta):=\frac{1}{\mathcal{V}_{R}} \frac{t r_{\mathcal{D}} H_{R} e^{-\beta H_{R}}}{t r_{\mathcal{D}} e^{-\beta H_{R}}}=\left(\frac{\pi^{2}}{30}-\frac{1}{480 \pi^{2}} \frac{\beta^{4}}{R^{4}}+O\left(e^{-4 \pi^{2} \frac{R}{\beta}}\right)\right) \frac{1}{\beta^{4}} .
$$

The corresponding result for of the Maxwell free field $F_{\mu \nu}$ is

$$
\mathcal{E}_{R}^{(F)}(\beta)=\left(\frac{\pi^{2}}{15}-\frac{1}{6} \frac{\beta^{2}}{R^{2}}+\frac{1}{4 \pi^{3}} \frac{\beta^{3}}{R^{3}}-\frac{11}{240 \pi^{2}} \frac{\beta^{4}}{R^{4}}+O\left(e^{-4 \pi^{2} \frac{R}{\beta}}\right)\right) \frac{1}{\beta^{4}} .
$$


Proof. The hermitian operators $H$ and $H(2 R)$ are unitarily equivalent due to Eq. (5.3). This leads to the fact that $\operatorname{tr}_{\mathcal{D}} q^{H(2 R)}$ and $\operatorname{tr}_{\mathcal{D}} H(2 R) q^{H(2 R)}$ do not depend on $R$. Then Eqs. (4.30) and (4.86) imply that in the two models under consideration we have

$$
\mathcal{E}_{R}^{(\varphi)}(\beta)=\frac{G_{4}\left(\frac{i \beta}{2 \pi R}\right)-\frac{1}{240}}{R \mathcal{V}_{R}}, \mathcal{E}_{R}^{(F)}(\beta)=\frac{2 G_{4}\left(\frac{i \beta}{2 \pi R}\right)-2 G_{2}\left(\frac{i \beta}{2 \pi R}\right)-\frac{11}{120}}{R \mathcal{V}_{R}} .
$$

Using further the relations

$$
G_{2}(\tau)=\frac{1}{\tau^{2}} G_{2}\left(\frac{-1}{\tau}\right)-\frac{i}{4 \pi \tau}, \quad G_{4}(\tau)=\frac{1}{\tau^{4}} G_{4}\left(\frac{-1}{\tau}\right)
$$

(which are special cases of (A.13) and (A.14)) we find

$$
\begin{gathered}
\mathcal{E}_{R}^{(\varphi)}(\beta)=\frac{1}{\beta^{4}}\left(8 \pi^{2} G_{4}\left(\frac{2 \pi i R}{\beta}\right)-\frac{\beta^{4}}{480 \pi^{2} R^{4}}\right) \\
\mathcal{E}_{R}^{(F)}(\beta)=\frac{1}{\beta^{4}}\left(16 \pi^{2} G_{4}\left(\frac{2 \pi i R}{\beta}\right)+\frac{4 \beta^{2}}{R^{2}} G_{2}\left(\frac{2 \pi i R}{\beta}\right)+\frac{\beta^{3}}{4 \pi^{3} R^{3}}-\frac{11 \beta^{4}}{240 \pi^{2} R^{4}}\right) .
\end{gathered}
$$

Finally, to obtain Eqs. (5.8) and (5.9) one should apply the expansion (A.16) implying that

$$
G_{2}\left(\frac{2 \pi i R}{\beta}\right)=-\frac{1}{24}+O\left(e^{-4 \pi^{2} \frac{R}{\beta}}\right), \quad G_{4}\left(\frac{2 \pi i R}{\beta}\right)=\frac{1}{240}+O\left(e^{-4 \pi^{2} \frac{R}{\beta}}\right) .
$$

Remark 5.2. In order to make comparison with the familiar expression for the black body radiation it is instructive to restore the dimensional constants $h$ and $c$ setting $H_{R}=\frac{h c}{R} H(2 R)$ (instead of (5.4)). The counterpart of (5.10) and (A.16) then reads

$$
\left\langle H_{R}\right\rangle_{q}=\frac{h c}{R}\left(G_{4}\left(\frac{i h c \beta}{R}\right)-E_{0}\right)=\frac{h c}{R} \sum_{n=1}^{\infty} \frac{n^{3} e^{-n \frac{h c \beta}{R}}}{1-e^{-n \frac{h c \beta}{R}}} .
$$

Each term in the infinite sum in the right hand side is a constant multiple of Plank's black body radiation formula for frequency

$$
\nu=n \frac{c}{R} \text {. }
$$

Thus, for finite $R$, there is a minimal frequency, $\frac{c}{R}$. Using the expansion in (5.14) one can also find an alternative integral derivation of the limit mean energy density $\mathcal{E}_{R}^{(\varphi)}(\beta)$ (5.10):

$$
\mathcal{E}_{R}^{(\varphi)}(\beta)=\frac{1}{2 \pi^{2} h^{3} c^{3} \beta^{4}} \sum_{n=1}^{\infty} \frac{\left(n \frac{h c \beta}{R}\right)^{3} e^{-n \frac{h c \beta}{R}}}{1-e^{-n \frac{h c \beta}{R}}} \frac{h c \beta}{R} \underset{R \rightarrow \infty}{\longrightarrow} \frac{\pi^{2}}{30 h^{3} c^{3} \beta^{4}}
$$


since the sum in the right hand side goes to the integral $\int_{0}^{\infty} \frac{t^{3} e^{-t}}{1-e^{-t}} d t=\frac{\pi^{4}}{15}$.

Remark 5.3. We observe that the constant $C$ in (5.7) in both considered models is equal to $\frac{c_{1}}{30 \pi^{2}}$, where $c_{1}$ is the coefficient to the $G_{4}-$ modular form in $\langle H\rangle_{q}$ (see Eq. (4.29)). If we use in the definition (5.5) of $H_{R}$ the Hamiltonian $H(2 R)+E_{0}^{\prime}$ instead of $H(2 R), \widetilde{H}_{R}:=\frac{H(2 R)+E_{0}^{\prime}}{R}$, then this will only reflect on the (nonleading) terms $c_{4} \frac{\beta^{4}}{R^{4}}$ in (5.8) (5.9) replacing them by $\frac{E_{0}^{\prime}-E_{0}}{2 \pi^{2}} \frac{\beta^{4}}{R^{4}}$, where $E_{0}$ is the "vacuum energy" for the corresponding models (i.e., $E_{0}$ is $\frac{1}{240}$ and $\frac{11}{120}$ for the fields $\varphi$ and $F_{\mu \nu}$, respectively).

\subsection{Infinite volume limit of the thermal correlation functions}

We shall study the $R \rightarrow \infty$ limit on the example of a free scalar field $\varphi$ in four dimensions.

Denote by $\varphi^{M}(x)$ (the canonically normalized) $D=4$ free massless scalar field with 2 -point function

$$
\left\langle 0\left|\varphi^{M}\left(x_{1}\right) \varphi^{M}\left(x_{2}\right)\right| 0\right\rangle=(2 \pi)^{-2}\left(x_{12}^{2}+i 0 x_{12}^{0}\right)^{-1}
$$

$\left(x_{12}=x_{1}-x_{2}, x_{12}^{2}=x_{12}^{2}-\left(x_{12}^{0}\right)^{2}\right)$. We define, in accord with Proposition 5.1, a finite volume approximation of its thermal correlation function by

$$
\left\langle\varphi^{M}\left(x_{1}\right) \varphi^{M}\left(x_{2}\right)\right\rangle_{\beta, R}:=\frac{\operatorname{tr}_{\mathcal{D}} \varphi^{M}\left(x_{1}\right) \varphi^{M}\left(x_{2}\right) e^{-\beta H_{R}}}{\operatorname{tr}_{\mathcal{D}} e^{-\beta H_{R}}}
$$

and will be interested in the thermodynamic limit,

$$
\left\langle\varphi^{M}\left(x_{1}\right) \varphi^{M}\left(x_{2}\right)\right\rangle_{\beta, \infty}:=\lim _{R \rightarrow \infty}\left\langle\varphi^{M}\left(x_{1}\right) \varphi^{M}\left(x_{2}\right)\right\rangle_{\beta, R} .
$$

Proposition 5.3. The limit (5.19) (viewed as a meromorphic function) is given by

$$
\begin{aligned}
& \left\langle\varphi^{M}\left(x_{1}\right) \varphi^{M}\left(x_{2}\right)\right\rangle_{\beta, \infty}=\frac{\sinh 2 \pi \frac{\left|\boldsymbol{x}_{12}\right|}{\beta}}{8 \pi \beta\left|\boldsymbol{x}_{12}\right|}\left(\cosh 2 \pi \frac{\left|\boldsymbol{x}_{12}\right|}{\beta}-\cosh 2 \pi \frac{x_{12}^{0}}{\beta}\right)^{-1} \\
& \left(\left|\boldsymbol{x}_{12}\right|:=\sqrt{\boldsymbol{x}_{12}^{2}} \equiv \sqrt{\left(x_{12}^{1}\right)^{2}+\left(x_{12}^{2}\right)^{2}+\left(x_{12}^{3}\right)^{2}}\right)
\end{aligned}
$$

We shall prove this statement by relating $\varphi^{M}(x)$ to the compact picture field $\varphi(\zeta, u)\left(\equiv \phi^{(1)}(\zeta, u)\right)$ whose thermal 2-point function was computed in Sect. 4.2.

First, we use Eq. (2.23) to express $\varphi^{M}(x)$ in terms of the $z$-picture field (corresponding to the $R$-depending chart (5.1))

$$
2 \pi \varphi^{M}(x)=\frac{1}{2 \omega\left(\frac{x}{2 R}\right)} \varphi_{R}(z(x ; R))
$$


(since $d z^{2}=\omega\left(\frac{x}{2 R}\right)^{-2} \frac{d x^{2}}{4}, \mathrm{cp} .(2.4)$ ). The factor $2 \pi$ in front of $\varphi^{M}$ accounts for the different normalization conventions for the $x$ - and $z$-picture fields (we have $\left\langle 0\left|\varphi\left(z_{1}\right) \varphi\left(z_{2}\right)\right| 0\right\rangle=\left(z_{12}^{2}\right)^{-1}$ instead of (5.17)).

As a second step we express $\varphi_{R}(z)-$ and thus $\varphi^{M}(x)$ - in terms of the compact picture field $\varphi_{R}(\zeta, u)$ :

$$
\begin{gathered}
\varphi_{R}(\zeta, u):=R e^{2 \pi i \zeta} \varphi\left(R e^{2 \pi i \zeta} u\right), \\
2 \pi \varphi^{M}(x)=\frac{1}{2 R\left|\omega\left(\frac{x}{2 R}\right)\right|} \varphi_{R}\left(\zeta\left(\frac{x}{2 R}\right), u\left(\frac{x}{2 R}\right)\right) .
\end{gathered}
$$

Here $\zeta$ and $u$ are determined as functions of $\frac{x}{2 R}$ from $e^{2 \pi i \zeta} u=\frac{z(x ; R)}{R}=z\left(\frac{x}{2 R}\right)$ $(z(x)$ is the transformation (2.3)); in deriving the second equation in (5.22) we have used the relation $e^{4 \pi i \zeta}=\frac{z(x ; R)^{2}}{R^{2}}=\overline{\omega\left(\frac{x}{2 R}\right)} \omega\left(\frac{x}{2 R}\right)^{-1}$.

Next we observe that $\varphi_{R}(\zeta, u)$ are mutually conjugate (for different $R$ ) just as $H(2 R)$ in Eq. (5.3). (To see this one can use an intermediate "dimensionless" coordinates $\widetilde{z}(x ; R)=\frac{z}{R}=z\left(\frac{x}{2 R}\right)$, which differs from (2.3) just by the dilation $(2 R)^{\Omega_{-1 D}}$.) It follows that its vacuum and thermal $2-$ point function with respect to the Hamiltonian $H(2 R)$ do not depend on $R$ and coincide with (4.20) (for $d=1$ ) and (4.22). Thus

$$
4 \pi^{2}\left\langle\varphi^{M}\left(x_{1}\right) \varphi^{M}\left(x_{2}\right)\right\rangle_{\beta, R}=\frac{p_{1}\left(\zeta_{12}+\alpha, \tau_{R}\right)-p_{1}\left(\zeta_{12}-\alpha, \tau_{R}\right)}{16 \pi R^{2}\left|\omega_{1} \omega_{2}\right| \sin 2 \pi \alpha}
$$

for $\omega_{k}=\omega\left(\frac{x_{k}}{2 R}\right), \zeta_{12}=\zeta\left(\frac{x_{1}}{2 R}\right)-\zeta\left(\frac{x_{2}}{2 R}\right), \cos 2 \pi \alpha=u\left(\frac{x_{1}}{2 R}\right) \cdot u\left(\frac{x_{2}}{2 R}\right), \tau_{R}=\frac{i \beta}{2 \pi R}$. In order to perform the $R \rightarrow \infty$ limit we derive the large $R$ behaviour of $\left|\omega_{k}\right|, \zeta_{12}$ and $\alpha$ :

$$
\begin{gathered}
2 \pi \zeta_{12}=\frac{x_{12}^{0}}{R}\left(1+O\left(\frac{\left\|x_{1}\right\|^{2}+\left\|x_{2}\right\|^{2}}{R^{2}}\right)\right), \quad 2 \pi \alpha=\frac{\left|x_{12}\right|}{R}\left(1+O\left(\frac{\left\|x_{1}\right\|^{2}+\left\|x_{2}\right\|^{2}}{R^{2}}\right)\right), \\
4\left|\omega_{k}\right|^{2}=1+O\left(\frac{\left\|x_{k}\right\|^{2}}{R^{2}}\right)
\end{gathered}
$$

$\left(\|x\|:=\sqrt{\left(x^{0}\right)^{2}+|x|^{2}}\right)$ following from

$$
\begin{aligned}
& \cos 2 \pi \zeta_{k}= \frac{1+\left(\frac{x_{k}}{2 R}\right)^{2}}{2\left|\omega_{k}\right|}, \quad \sin 2 \pi \zeta_{k}=\frac{x_{k}^{0}}{2 R\left|\omega_{k}\right|}, \quad \boldsymbol{u}=\frac{\boldsymbol{x}_{k}}{2 R\left|\omega_{k}\right|}, \quad u_{4}=\frac{1-\left(\frac{x_{k}}{2 R}\right)^{2}}{2 R\left|\omega_{k}\right|}, \\
& 4 \sin ^{2} \pi \alpha=\left(u_{1}-u_{2}\right)^{2}=\frac{\left|\boldsymbol{x}_{12}\right|^{2}}{R^{2}}\left(1+O\left(\frac{\left\|x_{1}\right\|^{2}+\left\|x_{2}\right\|^{2}}{R^{2}}\right)\right) .
\end{aligned}
$$


To evaluate the small $\tau_{R}$ (large $R$ ) limit of the difference of $p_{1}$-functions in (5.23) we use (A.11), (A.17) and (A.14) to deduce

$$
p_{1}(\zeta, \tau)=\frac{1}{\tau}\left(p_{1}\left(\frac{\zeta}{\tau}, \frac{-1}{\tau}\right)-2 \pi i \zeta\right) .
$$

Eq. (5.25) implies, on the other hand, that

$$
p_{1}\left(\frac{\zeta_{12} \pm \alpha}{\tau_{R}}, \frac{-1}{\tau_{R}}\right) \underset{R \rightarrow \infty}{\approx} p_{1}\left(\frac{x_{12}^{0} \pm\left|x_{12}\right|}{i \beta}, \frac{i 2 \pi R}{\beta}\right) \underset{R \rightarrow \infty}{\longrightarrow} \pi i \operatorname{coth}\left(\pi \frac{x_{12}^{0} \pm\left|x_{12}\right|}{\beta}\right) .
$$

Inserting (5.24)-(5.26) into (5.23) we complete the proof of (5.20) and hence of Proposition 5.3.

Remark 5.4. The physical thermal correlation functions should be, in fact, defined as distributions which amounts to giving integration rules around the poles. To do this one should view (5.20) as a boundary value of an analytic function in $x_{12}$ for $x_{12}^{0} \rightarrow x_{12}^{0}-i \varepsilon, \varepsilon>0, \varepsilon \rightarrow 0$ (cf. (5.17)). It is not difficult to demonstrate that the limit $\varepsilon \rightarrow+0$ and $R \rightarrow \infty$ in (5.19) commute. Using (5.25) we can also compute the $\frac{1}{R \beta}$ correction to $(5.20)$ :

$$
\left\langle\varphi^{M}\left(x_{1}\right) \varphi^{M}\left(x_{2}\right)\right\rangle_{\beta, R} \underset{R \gg \beta}{\approx}\left\langle\varphi^{M}\left(x_{1}\right) \varphi^{M}\left(x_{2}\right)\right\rangle_{\beta, \infty}-\frac{1}{4 \pi^{2} \beta R} .
$$

To obtain the Fourier expansion of the result we combine Eqs. (5.23) (5.24) with the $q$-series (4.26) and set (as in Remark 5.2)

$$
\frac{n}{R}=p, \quad \sum_{n=1}^{\infty} \frac{1}{R} f\left(\frac{n}{R} ; x, \beta\right) \underset{R \rightarrow \infty}{\longrightarrow} \int_{0}^{\infty} f(p ; x, \beta) d p .
$$

The result is

$$
\begin{gathered}
(2 \pi)^{2}\left\langle\varphi^{M}\left(x_{1}\right) \varphi^{M}\left(x_{2}\right)\right\rangle_{\beta, \infty}=\frac{1}{x_{12}^{2}+i 0 x_{12}^{0}}+ \\
+\frac{2}{\left|\boldsymbol{x}_{12}\right|} \int_{0}^{\infty} \frac{e^{-\beta p}}{1-e^{-\beta p}} \cos \left(p x_{12}^{0}\right) \sin \left(p\left|\boldsymbol{x}_{12}\right|\right) d p .
\end{gathered}
$$

To conclude: the conformal compactification $\bar{M}$ of Minkowski space $M$ plays a dual role.

On one hand, it can serve as a symmetric finite box approximation to $M$ in the study of finite temperature equilibrium states. In fact, any finite inverse temperature $\beta$ actually fixes a Lorentz frame (cf. [8]) so that the symmetry of a Gibbs state is described by the 7-parameter "Aristotelian group" of (3-dimensional) Euclidean motions and time translations. In the passage from $M$ to $\bar{M}$ the Euclidean group is deformed to the (stable) compact group of 4-dimensional rotations while the group of time translations is compactified to $U(1)$. Working throughout with the maximal (7-parameter) symmetry allows to write down simple explicit formulae for both finite $R$ and the "thermodynamic limit". 
On the other hand, taking $\bar{M}$ (and its universal cover, $\widetilde{M}=\mathbb{R} \times \mathbb{S}^{3}$ ) not as an auxiliary finite volume approximation but as a model of a static space-time, we can view $R$ as a (large but) finite quantity and use the above discussion as a basis for studding finite $R$ corrections to the Minkowski space formulae. It is a challenge from this second point of view to study the conformal symmetry breaking by considering massive fields in $\widetilde{M}$.

\section{Concluding Remarks}

Periodicity of (observable) GCI fields in the conformal time variable $\zeta$ suggests that their Gibbs (finite temperature) correlation functions should be (doubly periodic) elliptic functions in the conformal time differences with second period proportional to the (complexified) inverse absolute temperature. We give arguments (Theorem 3.5, Corollary 3.6) that this is indeed the case in a GCI Wightman theory. Explicit constructions are presented of elliptic 2-point functions of free fields in an even number of space-time dimensions.

If a field $\psi(\zeta, u)$ of dimension $d$ and its conjugate satisfy the strong locality property $(2.22)$, i.e., if

$$
\left(\cos 2 \pi \zeta_{12}-u_{1} \cdot u_{2}\right)^{N}\left[\psi\left(\zeta_{1}, u_{1}\right) \psi^{*}\left(\zeta_{2}, u_{2}\right)-(-1)^{2 d} \psi^{*}\left(\zeta_{2}, u_{2}\right) \psi\left(\zeta_{1}, u_{1}\right)\right]=0
$$

for $N \geqslant N_{\psi}$ then the Gibbs 2-point function $\left\langle\psi\left(\zeta_{1}, u_{1}\right) \psi^{*}\left(\zeta_{2}, u_{2}\right)\right\rangle_{q}$ has exactly two poles in a fundamental domain, centred around the origin of the $\zeta_{12}$ plane, of leading order $N_{\psi}$ at the points

$$
\zeta_{12}= \pm \alpha \quad \text { for } \quad u_{1} \cdot u_{2}=\cos 2 \pi \alpha, \quad 0 \leqslant \alpha<\frac{1}{2} .
$$

For a rank $\ell$ symmetric tensor field $\psi$ of dimension $d$ the integer $N_{\psi}$ coincides with $d+\ell$; for an irreducible spin-tensor field in $D=4$, of $S(U(2) \times U(2))$-weight $\left(d ; j_{1}, j_{2}\right)$, we have $N_{\psi}=d+j_{1}+j_{2}$.

The conformal energy mean value in an equilibrium Gibbs state (with suitably shifted vacuum energy) appears as a superposition of modular forms of different weights. Postulating this property for the photon energy (associated with the Maxwell stress tensor $F$ ) requires including (non-physical) gauge degrees of freedom (otherwise, the non-modular term $-2 G_{2}(\tau)$ contributes to (4.86)). The result is then a modular form of weight 4 (Sect. 4.4, Eq. (4.90)). The same is true for the free massless scalar field for $D=4$, while the energy mean of a $d=\frac{3}{2}$ Weyl field is a superposition of modular forms (4.68) of weight 4 and 2 (and level $\Gamma_{\theta}$ - see Appendix A, Eq. (A.15) and the text following it). The question arises whether relaxing the condition of Wightman positivity one cannot find an (indefinite metric) interacting Weyl field model whose energy mean value is a (homogeneous) modular form of weight four (as suggested by the study of chiral conformal models in $1+1$ space-time dimension). More generally, the role of modular invariance in higher dimensional conformal field models still awaits its full understanding. 


\section{Acknowledgments}

The authors' interest in the modular properties of energy distributions in higher dimensional conformal field theory was stimulated by an early suggestion of Maxim Kontsevitch. Discussions with Petko Nikolov are also gratefully acknowledged. We thank Seif Randjbar-Daemi and the Abdus Salam International Centre for Theoretical Physics in Trieste for invitation and support during a late stage of this work. Discussions with Detlev Buchholz in Göttingen led to including the addition of the present Sect. 5. We acknowledge partial support of the Alexander von Humboldt Foundation and the hospitality of the Institut für Theoretische Physik der Universität Göttingen during the final stage of this work. Our work is supported in part by the Research Training Network within the Framework Programme 5 of the European Commission under contract HPRN-CT-2002-00325 and by the Bulgarian National Council for Scientific Research under contract PH-1406.

\section{Appendix A. Basic Elliptic Functions}

In this Appendix we define the basic elliptic functions used in the paper and list their properties and relations with the conventional functions.

Recall that an elliptic function $f(\zeta)$ is a meromorpic function on $\mathbb{C}(\ni \zeta)$ which is doubly periodic. Its periods can be chosen (after rescalling by a nonzero complex constant) to be 1 and $\tau$ with $\tau \in \mathfrak{H}\left(:=\left\{\tau^{\prime} \in \mathbb{C}: \operatorname{Im} \tau^{\prime}>0\right\}\right)$. Thus, $f(\zeta)=f(\zeta+m+n \tau)$ for $m, n \in \mathbb{Z}$ and hence, $f$ is completely determined by its values in the fundamental domain $\mathfrak{D}:=\{\zeta \in \mathbb{C}: \zeta=\lambda+\mu \tau, 0 \leqslant \lambda, \mu<1\}$. By the Liouville's theorem, $f(\zeta)$ should have at least one pole in $\mathfrak{D}$ if it is nonconstant: otherwise it will be bounded nonconstant entire function in $\zeta$ which is not possible. Integrating $f(\zeta)$ and $\frac{f^{\prime}(\zeta)}{f(\zeta)}$ over the boundary $\partial \mathfrak{D}$ (or, over the shifted $\partial \mathfrak{D}+c$, if necessary) we conclude in addition (by the Cauchy theorem on one hand, and the double periodicity, on the other) that: $(i)$ the sum of the residues of the simple poles of $f$ lying in $\mathfrak{D}$ is zero and, $(i i)$ the sum of multiplicities of all zeros minus the sum of multiplicities of all poles of $f$ in $\mathfrak{D}$ is also zero. In particular, $f$ cannot have just one simple pole in $\mathfrak{D}$. Therefore, if the singular part of $f$ in $\mathfrak{D}$ has the form:

$$
\sum_{k=1}^{K} \sum_{s=1}^{S} N_{k, s} \frac{1}{\left(\zeta-\zeta_{s}\right)^{k}}
$$

for some $K, S \in \mathbb{N}, N, N_{s, k} \in \mathbb{C}, \zeta_{s} \in \mathfrak{D}(k=1, \ldots, K, s=1, \ldots, S)$, then $f$ can be represented in a finite sum:

$$
f(\zeta)=N+\sum_{k=1}^{K} \sum_{s=1}^{S} N_{k, s} p_{k}\left(\zeta-\zeta_{s}, \tau\right)
$$

where $p_{k}(\zeta, \tau)$ are, roughly speaking, equal to:

$$
p_{k}(\zeta, \tau):=\sum_{m, n \in \mathbb{Z}} \frac{1}{(\zeta+m+n \tau)^{k}} .
$$


The series (A.3) are absolutely convergent for $k \geqslant 3$ and

$$
p_{k+1}(\zeta, \tau)=-\frac{1}{k}\left(\partial_{\zeta} p_{k}\right)(\zeta, \tau) .
$$

For $k=1,2$ one should specialize the order of summation or, alternatively, add regularizing terms. In such a way we arrive at the standard Weierstrass functions [12]:

$$
\begin{aligned}
& \mathfrak{Z}(\zeta, \tau)=\frac{1}{\zeta}+\sum_{\substack{(m, n) \in \\
\in \mathbb{Z}^{2} \backslash\{(0,0)\}}}\left[\frac{1}{\zeta+m \tau+n}-\frac{1}{m \tau+n}+\frac{\zeta}{(m \tau+n)^{2}}\right], \\
& \wp(\zeta, \tau)=\frac{1}{\zeta^{2}}+\sum_{\substack{(m, n) \in \\
\in \mathbb{Z}^{2} \backslash\{(0,0)\}}}\left[\frac{1}{(\zeta+m \tau+n)^{2}}-\frac{1}{(m \tau+n)^{2}}\right] .
\end{aligned}
$$

Thus $\mathfrak{Z}(\zeta, \tau)$ and $\wp(\zeta, \tau)$ are odd and even meromorphic functions in $\zeta$, respectively, and

$$
\left(\partial_{\zeta} \mathfrak{Z}\right)(\zeta, \tau)=-\wp(\zeta, \tau), \quad\left(\partial_{\zeta} \wp\right)(\zeta, \tau)=-2 p_{3}(\zeta, \tau)
$$

Since $p_{3}(\zeta, \tau)$ is elliptic it then follows that $\wp(\zeta, \tau)$ is also elliptic. The function $\mathfrak{Z}(\zeta, \tau)$ cannot be elliptic (by the property $(i)$ above) and, in fact,

$$
\begin{aligned}
& \mathfrak{Z}(\zeta+1, \tau)=\mathfrak{Z}(\zeta, \tau)-8 \pi^{2} G_{2}(\tau), \\
& \mathfrak{Z}(\zeta+\tau, \tau)=\mathfrak{Z}(\zeta, \tau)-8 \pi^{2} G_{2}(\tau) \tau-2 \pi i
\end{aligned}
$$

where

$$
G_{2 k}(\tau)=\frac{(2 k-1) !}{2(2 \pi i)^{2 k}}\left\{\sum_{n \in \mathbb{Z} \backslash\{0\}} \frac{1}{n^{2 k}}+\sum_{m \in \mathbb{Z} \backslash\{0\}} \sum_{n \in \mathbb{Z}} \frac{1}{(m \tau+n)^{2 k}}\right\}
$$

$(k=1,2, \ldots)$ are the $G$-modular functions also playing central role in this work. Hence, $\mathfrak{Z}(\zeta, \tau)$ and $\wp(\zeta, \tau)$ are possible candidates for $p_{1}$ and $p_{2}$ and they are indeed used as basic functions in [38]. As we have explained in the introduction we prefer to work with (anti)periodic function in $\zeta$ with period 1 and on the other hand, to preserve the relation (A.4) for all $k \in \mathbb{N}$ so that this naturally fixes

$$
\begin{aligned}
& p_{1}(\zeta, \tau):=\mathfrak{Z}(\zeta, \tau)+8 \pi^{2} G_{2}(\tau) \zeta, \\
& p_{2}(\zeta, \tau):=\wp(\zeta, \tau)-8 \pi^{2} G_{2}(\tau) .
\end{aligned}
$$

For $k>1$, the above introduced $G_{2 k}(\tau)$ are modular forms of weight $2 k$ (and level 1):

$$
\frac{1}{(c \tau+d)^{2 k}} G_{2 k}\left(\frac{a \tau+b}{c \tau+d}\right)=G_{2 k}(\tau) \quad \text { for } \quad\left(\begin{array}{ll}
a & b \\
c & d
\end{array}\right) \in S L(2, \mathbb{Z}),
$$

while for $k=1$ we have instead

$$
\frac{1}{(c \tau+d)^{2}} G_{2}\left(\frac{a \tau+b}{c \tau+d}\right)=G_{2}(\tau)+\frac{i c}{4 \pi(c \tau+d)} \text {. }
$$


In applications to CFT there appear more general modular forms like

$$
F(\tau):=2 G_{2}(\tau)-G_{2}\left(\frac{\tau+1}{2}\right)
$$

which is invariant under the index 2 subgroup $\Gamma_{\theta}$ of $S L(2, \mathbb{Z})$ generated by $S$ and $T^{2}$ where $S$ is given by (1.2) and $T=\left(\begin{array}{ll}1 & 1 \\ 0 & 1\end{array}\right)$. We note that the normalization factor in the definition of the modular forms $G_{2 k}(\tau)$ (A.10) is chosen so that the coefficient to $q$ in their Fourier expansion is 1 . Then one finds that all Fourier coefficients (except the constant term) are positive integers:

$$
G_{2 k}(\tau)=-\frac{B_{2 k}}{4 k}+\sum_{n=1}^{\infty} \frac{n^{2 k-1}}{1-q^{n}} q^{n}=\frac{1}{2} \zeta(1-2 k)+\sum_{n=1}^{\infty} \sigma_{2 k-1}(n) q^{n}
$$

where $\sigma_{l}(n)=\sum_{r \mid n} r^{l}$ (sum over all positive divisors $r$ of $n$ ), $B_{l}$ are the Bernoulli numbers, and $\zeta(s)$ is the Riemann $\zeta$-function.

Let us mention also the modular transformation properties of the Weierstrass functions (A.6) and (A.5)

$$
\begin{gathered}
\frac{1}{c \tau+d} \mathfrak{Z}\left(\frac{\zeta}{c \tau+d}, \frac{a \tau+b}{c \tau+d}\right)=\mathfrak{Z}(\zeta, \tau), \\
\frac{1}{(c \tau+d)^{2}} \wp\left(\frac{\zeta}{c \tau+d}, \frac{a \tau+b}{c \tau+d}\right)=\wp(\zeta, \tau) .
\end{gathered}
$$

Thus, our $p_{1}(\zeta, \tau)(\mathrm{A} .17)$ and $p_{2}(\zeta, \tau)$ (A.18) will obey inhomogeneous modular transformation laws (as in the example of Eq. (5.25)). (This is the price for preserving the periodicity property for $\zeta \mapsto \zeta+1$.)

We will use also the Jacobi $\vartheta$-functions [25] [37]:

$$
\begin{gathered}
\vartheta(\zeta, \tau):=\sum_{n=-\infty}^{\infty} e^{\pi i\left(n^{2} \tau+2 n \zeta\right)} \equiv \vartheta_{00}(\zeta, \tau), \\
\vartheta_{\lambda \kappa}(\zeta, \tau):=e^{\pi i \tau \frac{\lambda^{2}}{4}+\pi i \lambda\left(\zeta+\frac{\kappa}{2}\right)} \vartheta\left(\zeta+\frac{\lambda \tau+\kappa}{2}, \tau\right)
\end{gathered}
$$

for $\kappa, \lambda=0,1$, which have the following properties (for $\kappa, \lambda=0,1$ ):

$$
\begin{aligned}
\vartheta_{\lambda \kappa}(\zeta+m \tau+n, \tau) & =(-1)^{m \kappa+n \lambda} e^{-\pi i\left(m^{2} \tau+2 m \zeta\right)} \vartheta_{\lambda \kappa}(\zeta, \tau), \\
\vartheta_{\lambda \kappa}(-\zeta, \tau) & =(-1)^{\lambda \kappa} \vartheta_{\lambda \kappa}(\zeta, \tau) \\
\vartheta_{\lambda \kappa}(\zeta, \tau) & =0 \Leftrightarrow \quad \Leftrightarrow \in\left(\mathbb{Z}+\frac{1-\lambda}{2}\right) \tau+\mathbb{Z}+\frac{1-\kappa}{2}
\end{aligned}
$$

(Eqs. (A.21) and (A.22) are first proven for the series (A.19) and then for the other functions (A.20); Eq. (A.23) follows from Eqs. (A.22) and (A.20) since the first one means that $\vartheta_{11}(\zeta, \tau)$ is odd in $\zeta$.) We are using in Sect. 3.2 the fact that the odd $\vartheta$-function, $\vartheta_{11}$, can be written in the form

$$
\vartheta_{11}(\zeta, \tau)=-2 \sum_{n=0}^{\infty}(-1)^{n} e^{i \pi \tau\left(n+\frac{1}{2}\right)^{2}} \sin (2 n+1) \pi \zeta .
$$


Returning to our set $\left\{p_{k}(\zeta, \tau)\right\}$ of basic elliptic functions we can rewrite (the qausielliptic) $p_{1}(\zeta, \tau)$ as

$$
\begin{aligned}
p_{1}(\zeta, \tau) & =\lim _{N \rightarrow \infty} \sum_{n=-N}^{N} \pi \operatorname{cotg}[\pi(\zeta+n \tau)]= \\
& =\sum_{n=-\infty}^{\infty}\{\pi \operatorname{cotg}[\pi(\zeta+n \tau)]+i \pi \operatorname{sgn}(n)\}= \\
& =\lim _{M \rightarrow \infty} \lim _{N \rightarrow \infty} \sum_{m=-M}^{M} \sum_{n=-N}^{N} \frac{1}{\zeta+m+n \tau},
\end{aligned}
$$

where $\operatorname{sgn}(n):=\frac{|n|}{n}$ for $n \neq 0$ and $\operatorname{sgn}(0):=0$. Indeed, first note that the second sum in Eq. (A.25) is absolutely convergent since the absolute value of the summand has a behaviour as $e^{-\pi|n| \operatorname{Im} \tau}$. Then Eq. (A.26) follows from Euler's identity:

$$
\frac{\pi \cos ^{1-\lambda} \pi \zeta}{\sin \pi \zeta}=\lim _{N \rightarrow \infty} \sum_{n=-N}^{N} \frac{(-1)^{n \lambda}}{\zeta+n} \quad(\lambda=0,1) .
$$

Finally, to obtain the first Eq. (A.25) we take the difference between both sides and observe that it is an elliptic function in $\zeta$, in accord to Eqs. (A.9) and (A.11). On the other hand, this difference is regular in $\zeta$ in the fundamental domain $\mathfrak{D}$, because of Eqs. (A.5) and (A.26), so that it is a constant which is actually zero since it is obviously an odd function in $\zeta$.

Eq. (A.25) is closely related to the general form of the elliptic correlation functions arising in the free field GCI models according to Theorem 4.1 (see Eq. (4.6)). In view of the more general situation of the "grand canonical" corelation functions in Remark 4.1 (Eq. (4.11)) we are led to introduce for $\kappa, \lambda=0,1, \tau \in \mathfrak{H}$, $\zeta \in \mathbb{C} \backslash(\mathbb{Z} \tau+\mathbb{Z})$ and $\mu \in \mathbb{R}$

$$
p_{1}^{\kappa, \lambda}(\zeta, \tau, \mu)=\sum_{n=-\infty}^{\infty}\left[\frac{\pi \cos ^{1-\lambda}[\pi(\zeta+n \tau)]}{\sin [\pi(\zeta+n \tau)]}+i \pi(1-\lambda) \operatorname{sgn}(n)\right] e^{\pi i n(2 \mu+\kappa)} .
$$

For $|n| \gg 0$ the absolute value of the summand in the above series will have a behaviour as $e^{-\pi|n| \operatorname{Im} \tau}$ and therefore, the series is convergent for every $\zeta \in$ $\mathbb{C} \backslash(\mathbb{Z} \tau+\mathbb{Z}), \mu \in \mathbb{R}$ and $\tau \in \mathfrak{H}$. It then follows that

$$
\begin{aligned}
& p_{1}^{\kappa, \lambda}(\zeta+\tau, \tau, \mu)=(-1)^{\kappa} e^{-2 \pi i \mu} p_{1}^{\kappa, \lambda}(\zeta, \tau, \mu)-\pi i(1-\lambda)\left(1+e^{-\pi i(2 \mu+\kappa)}\right), \\
& p_{1}^{\kappa, \lambda}(\zeta+1, \tau, \mu)=(-1)^{\lambda} p_{1}^{\kappa, \lambda}(\zeta, \tau, \mu) .
\end{aligned}
$$

In the case of $\kappa=\lambda=0$ we will simplify the notation setting

$$
p_{1}(\zeta, \tau, \mu):=p_{1}^{00}(\zeta, \tau, \mu) .
$$


Proposition A.1. The functions $p_{1}^{\kappa, \lambda}(\zeta, \tau, \mu)$ (A.28) $(\kappa, \lambda=0,1)$ have an analytic extension to meromorphic functions in $(\zeta, \tau, \mu) \in \mathbb{C} \times \mathfrak{H} \times \mathbb{C}$ given for $\mu+\frac{\kappa}{2} \in \mathbb{R} \backslash \mathbb{Z}$, by

$$
p_{1}^{\kappa, \lambda}(\zeta, \tau, \mu)=\frac{\left(\partial_{\zeta} \vartheta_{11}\right)(0, \tau)}{\vartheta_{1-\lambda 1-\kappa}(\mu, \tau)} \frac{\vartheta_{1-\lambda 1-\kappa}(\zeta+\mu, \tau)}{\vartheta_{11}(\zeta, \tau)}-(1-\lambda) \pi \operatorname{cotg} \pi\left(\mu+\frac{\kappa}{2}\right) .
$$

They are regular for all $\mu \in \mathbb{R}$ and

$$
p_{1}(\zeta, \tau, 0)=\frac{\left(\partial_{\zeta} \vartheta_{11}\right)(\zeta, \tau)}{\vartheta_{11}(\zeta, \tau)} \equiv p_{1}(\zeta, \tau)
$$

$p_{1}(\zeta, \tau)$ being defined by Eq. (A.11).

Proof. Let $\mu \in \mathbb{R}$ and take the difference $\Delta(\zeta, \tau, \mu)$ between the left and right hand sides of Eq. (A.31). From the properties (A.21)-(A.23) and (A.29) we find that

$$
\Delta(\zeta+m \tau+n, \tau, \mu)=(-1)^{m \kappa+n \lambda} e^{-2 \pi i m \mu} \Delta(\zeta, \tau, \mu) .
$$

(Note that the second ratio in Eq. (A.31) is chosen to obey the the quasiperiodicity property (A.33) and its pole coefficient at $\zeta=0$ is canceled by the first ratio.) On the other hand, $\Delta(\zeta, \tau, \mu)$ is analytic in $\zeta$, for fixed $\tau$ and $\mu$, outside the lattice $\mathbb{Z} \tau+\mathbb{Z} \subset \mathbb{C}$ and since it is also regular at the origin $\zeta=0$, Eq. (A.33) then implies that $\Delta(\zeta, \tau, \mu)$ is an entire bounded function in $\zeta$. By the Liouville's theorem we conclude that $\Delta(\zeta, \tau, \mu)$ does not depend on $\zeta$ and it is actually zero, again by Eq. (A.33).

Eq. (A.32) follows in the same way from Eqs. (A.8), (A.9) and (A.29). (The constant here is fixed by the behaviour for $\zeta \rightarrow 0$.)

For $k=1,2, \ldots$ we set

$$
\begin{aligned}
& p_{k+1}^{\kappa, \lambda}(\zeta, \tau, \mu)=-\frac{1}{k} \partial_{\zeta} p_{k}^{\kappa, \lambda}(\zeta, \tau, \mu), \quad p_{k}(\zeta, \tau, \mu):=p_{k}^{00}(\zeta, \tau, \mu), \\
& p_{k+1}^{\kappa, \lambda}(\zeta, \tau)=p_{k}^{\kappa, \lambda}(\zeta, \tau, 0)\left(=-\frac{1}{k} \partial_{\zeta} p_{k}^{\kappa, \lambda}(\zeta, \tau)\right), \quad p_{k}(\zeta, \tau):=p_{k}^{00}(\zeta, \tau) .
\end{aligned}
$$

Proposition A.2. Every function $p_{k}^{\kappa, \lambda}(\zeta, \tau, \mu)$, for $k=2,3, \ldots$, is uniquely characterized by the conditions:

(a) $p_{k}^{\kappa, \lambda}(\zeta, \tau, \mu)$ is a meromorphic function in $(\zeta, \tau, \mu) \in \mathbb{C} \times \mathfrak{H} \times \mathbb{C}$ and for real $\mu$, and for all $\tau \in \mathfrak{H}, k=1,2, \ldots, \kappa, \lambda=0,1$, it has exactly one pole in $\zeta$ at 0 of order $k$ and residue 1 in the domain $\{\alpha \tau+\beta: \alpha, \beta \in[0,1)\} \subset \mathbb{C}$;

(b) $p_{k}^{\kappa, \lambda}(\zeta+1, \tau, \mu)=(-1)^{\lambda} p_{k}^{\kappa, \lambda}(\zeta, \tau, \mu)$;

(c) $p_{k}^{\kappa, \lambda}(\zeta+\tau, \tau, \mu)=e^{-\pi i(2 \mu+\kappa)} p_{k}^{\kappa, \lambda}(\zeta, \tau, \mu)$.

It also obeys the property

(d) $p_{k}^{\kappa, \lambda}(-\zeta, \tau, \mu)=(-1)^{k} p_{k}^{\kappa, \lambda}(\zeta, \tau, \mu)$. 
The function $p_{1}^{\kappa, \lambda}(\zeta, \tau, \mu)$ can be fixed by the condition (d) and the relation (A.34) connecting it with the function $p_{2}^{\kappa, \lambda}(\zeta, \tau, \mu)$.

For $\mu \in \mathbb{R}$ we have the series representation

$$
p_{k}^{\kappa, \lambda}(\zeta, \tau, \mu)=\sum_{m, n \in \mathbb{Z}} \frac{e^{\pi i m(2 \mu+\kappa)} e^{\pi i n \lambda}}{(\zeta+m \tau+n)^{k}},
$$

which is absolutely convergent for $k \geqslant 3$ and $\zeta \in \mathbb{C} \backslash(\mathbb{Z} \tau+\mathbb{Z})$, and for $k=1,2$ the sum should be taken first in $n$ for $|n| \leqslant N$ as $N \rightarrow \infty$ and then in $m$ for $|m| \leqslant M \rightarrow \infty$.

Proof. Clearly the of functions defined by Eqs. (A.31) and (A.34) satisfy the conditions $(a)-(d)$ except the case of $k=1$ in $(c)$. By the argument used in the proof of Proposition A.1 it follows that $(a)-(c)$ uniquely determine the functions $p_{k}^{\kappa, \lambda}(\zeta, \tau, \mu)$ for $k \geqslant 2$. The relation (A.34) fixes the function $p_{1}^{\kappa, \lambda}(\zeta, \tau, \mu)$ up to an additive constant which is determined by the condition $(d)$.

The derivation of Eq. (A.36) is based on (A.28) and (A.27).

Eq. (A.36) implies that:

$$
\begin{aligned}
& p_{k}^{01}(\zeta, \tau)=2^{1-k} p_{k}\left(\frac{\zeta}{2}, \frac{\tau}{2}\right)-p_{k}(\zeta, \tau) . \\
& p_{k}^{10}(\zeta, \tau)=p_{k}(\zeta, 2 \tau)-p_{k}(\zeta, \tau) \\
& p_{k}^{11}(\zeta, \tau)=2^{1-k} p_{k}\left(\frac{\zeta}{2}, \frac{\tau+1}{2}\right)-p_{k}(\zeta, \tau) .
\end{aligned}
$$

\section{Appendix B. Proof of Proposition 3.4}

We begin by recalling a basic fact of the theory of formal power series

Fact B.1. Let $R$ be a commutative ring with unit and $a(q)=1+\sum_{n=1}^{\infty} a_{n} q^{n} \in$ $R \llbracket q \rrbracket$ be an infinite formal power series in a single variable $q$. Then $a(q)$ is invertible in $R \llbracket q \rrbracket$, i.e., there exists unique $b(q)=\sum_{n=0}^{\infty} b_{n} q^{n} \in R \llbracket q \rrbracket$ such that $a(q) b(q)=1$. Moreover, $b_{0}=1$ and if $a(q)$ is a complex series that is absolutely convergent and nonzero for $|q|<\lambda$ then $b(q)$ is absolutely convergent for $|q|<\lambda^{-1}$.

Proof. Noting that $\left(1+\sum_{n=1}^{\infty} a_{n} q^{n}\right)\left(\sum_{n=0}^{\infty} b_{n} q^{n}\right)=b_{0}+\sum_{n=1}^{\infty}\left(b_{n}+\sum_{k=0}^{n-1} a_{n-k} b_{k}\right) q^{n}$ one can inductively determine $b_{n}$ starting with $b_{0}=1$. If $a(q)$ is absolutely convergent and nonzero for $|q|<\lambda$ then $b(q)$ will be the Taylor series of an analytic function for $|q|<\lambda^{-1}$ so that it will be absolutely convergent there.

Continuing with the proof of the statement $(a)$ of Proposition 3.4 we note first that $\Theta_{12}$ is obtained from (3.13) (see also (1.11) (1.12)) as a formal power series in $q$ with coefficients that are polynomials, say $\Theta_{12}^{(n)}$, in $e^{ \pm \pi i \zeta_{12}}$ and $e^{ \pm \pi i \alpha}$. Thus the 
coefficient in $\Theta_{12}^{(n)}$ to $e^{m \pi i \zeta_{12}}$ (for $|m| \leqslant n$ ) will be an even trigonometric polynomial in $\alpha$ with period 1 (since $\Theta_{12}$, as an analytic function, is even and periodic with period 1 in $\zeta_{12}$ as well as in $\alpha$, according to Eqs. (A.21) and (A.22)) and hence, $\Theta_{12}^{(n)}$ is a polynomial in $\cos 2 \pi \alpha=u_{1} \cdot u_{2} \in \mathbb{C}\left[u_{1}, u_{2}\right]$. Then considering $\Theta_{12}^{(n)}$ as a polynomial in $\cos 2 \pi \alpha$ we find in the same way that its coefficients are polynomials in $\cos 2 \pi \zeta_{12}$. Summarizing, we have $\Theta_{12} \in \mathbb{C}\left[e^{ \pm 2 \pi i \zeta_{1}}, u_{1} ; e^{ \pm 2 \pi i \zeta_{2}}, u_{2}\right]$. To prove next Eq. (3.15) we observe that $\Theta_{12}^{(n)}$ is a polynomial in $\cos 2 \pi \zeta_{12}$ (with polynomial coefficients in $\cos 2 \pi \alpha$ ) which is zero for $\cos 2 \pi \zeta_{12}=\cos 2 \pi \alpha$ (since $\Theta_{12}=0$ for $\left.\zeta_{12}= \pm \alpha\right)$. It then follows that $\frac{\Theta_{12}^{(n)}}{4 \sin \pi \zeta_{+} \sin \pi \zeta_{-}} \equiv \frac{\Theta_{12}^{(n)}}{2\left(\cos 2 \pi \alpha-\cos 2 \pi \zeta_{12}\right)}$ is again a polynomial in $\cos 2 \pi \zeta_{12}$ and $\cos 2 \pi \alpha$. This and the second equality in (3.13) prove Eq. (3.15). Since $\Theta_{12}$ is even in both, $\zeta_{12}$ and $\alpha$, we have the symmetry $\Theta_{12}=\Theta_{21}$.

Now the proof of the first part of Proposition $3.4(b)$ follows from Fact B.1, Eq. (3.15) and the existence in $\mathbb{C} \llbracket e^{ \pm 2 \pi i \zeta_{1}}, u_{1} \rrbracket_{+} \llbracket e^{ \pm 2 \pi i \zeta_{2}}, u_{2} \rrbracket_{+}$of the inverse:

$$
\begin{aligned}
\frac{1}{\sin \pi \zeta_{+} \sin \pi \zeta_{-}} & =\frac{4 e^{-2 \pi i \zeta_{12}}}{1-2 \cos (2 \pi \alpha) e^{-2 \pi i \zeta_{12}}+e^{-4 \pi i \zeta_{12}}}= \\
& =4 \sum_{n=0}^{\infty} C_{n}^{1}(\cos 2 \pi \alpha) e^{-(n+1) \pi i \zeta_{12}}
\end{aligned}
$$

where $C_{n}^{k}(t)$ are the Gegenbauer polynomials already used in Sect. 4.2.

Continuing with the proof of Proposition $3.4(c)$ we note first that the symmetry of $\Omega$ follows from that of $\Theta_{j k}$. To obtain Eq. (3.17) one first derives for $m \in \mathbb{Z}$ :

$$
\Theta\left(\zeta_{12}+m \tau ; u_{1}, u_{2}\right)=e^{-2 \pi i\left(m^{2} \tau+2 m \zeta_{12}\right)} \Theta\left(\zeta_{12} ; u_{1}, u_{2}\right)
$$

using Eqs. (3.13) and (A.12). Then we have for $\lambda_{1}, \ldots, \lambda_{n-1} \in \mathbb{Z}$ :

$$
\begin{aligned}
& \Omega\left(\zeta_{12}+\lambda_{1} \tau, \ldots, \zeta_{n-1 n}+\lambda_{n-1} \tau ; u_{1}, \ldots, u_{n} ; \tau\right)= \\
& =\prod_{1 \leqslant l<m \leqslant n} \Theta\left(\sum_{j=l}^{m-1}\left(\zeta_{j j+1}+\lambda_{j} \tau\right) ; u_{l}, u_{m}\right)= \\
& =\exp \left\{-2 \pi i \sum_{1 \leqslant l \leqslant m \leqslant n-1}\left[\left(\sum_{j=l}^{m} \lambda_{j}\right)^{2} \tau+2\left(\sum_{j=l}^{m} \lambda_{j}\right)\left(\sum_{j=l}^{m} \zeta_{j j+1}\right)\right]\right\} \times \\
& \quad \times \Omega\left(\zeta_{12}, \ldots, \zeta_{n-1} ; u_{1}, \ldots, u_{n} ; \tau\right)
\end{aligned}
$$

so that expanding the sums in the latter exponent we arrive at Eq. (3.17) with a positive definite integral matrix $\left\{A_{j k}^{(n)}\right\}_{j, k=1}^{n-1}$.

To prove the Proposition $3.4(d)$ let us write (following [25]):

$$
F\left(\zeta_{12}, \ldots, \zeta_{n-1 n} ; \tau\right)=\sum_{\left(m_{1}, \ldots, m_{n-1}\right) \in \mathbb{Z}^{n-1}} F_{m_{1} \ldots m_{n-1}}^{\prime}(q) e^{i \pi \sum_{k=1}^{n-1} m_{k} \zeta_{k} k+1}
$$

where $F_{m_{1} \ldots m_{n-1}}^{\prime}(q)$ are infinite formal power series in $q^{\frac{1}{2}}$ (with coefficients in the algebra $R$ ). Then the properties (3.17) combined with the expansion (B.5) implies 
that $F_{m_{1} \ldots m_{n-1}}^{\prime}(q)$ are nonzero if $m_{k}+\varepsilon_{k}^{(1)}=0 \bmod 2$. Therefore, we can rewrite the expansion (B.5) in the form:

$$
F\left(\zeta_{12}, \ldots, \zeta_{n-1 n} ; \tau\right)=\sum_{\nu_{1} \in \mathbb{Z}+\frac{\varepsilon_{1}^{(1)}}{2}} \ldots \sum_{\substack{\nu_{n-1} \in \mathbb{Z}+\frac{\varepsilon_{n-1}^{(1)}}{2} \\ F_{\nu_{1} \ldots \nu_{n-1}}}}(q) e^{2 \pi i \sum_{k=1}^{n-1} \nu_{k} \zeta_{k k+1}}
$$

$\left(F_{\nu_{1} \ldots \nu_{n-1}}(q) \in R \llbracket q^{\frac{1}{2}} \rrbracket\right)$. Now combining the expansion (B.5) with the property (3.18) we obtain:

$$
F_{\underline{\nu}+2 N A^{(n)}(\underline{\lambda})}(q)=(-1)^{\underline{\lambda} \cdot \underline{\varepsilon}^{(2)}} q^{N \underline{\lambda} \cdot A^{(n)}(\underline{\lambda})-\underline{\nu} \cdot \underline{\lambda}} F_{\underline{\nu}}(q)
$$

where $\underline{\nu}:=\left(\nu_{1}, \ldots, \nu_{n-1}\right), \underline{\lambda}:=\left(\lambda_{1}, \ldots, \lambda_{n-1}\right), \underline{\varepsilon}^{(k)}:=\left(\varepsilon_{1}^{(k)}, \ldots, \varepsilon_{n-1}^{(k)}\right), A^{(n)}(\underline{\lambda}):=$ $\left(\sum_{k=1}^{n-1} A_{j k}^{(n)} \lambda_{k}\right)_{j=1}^{n-1}$ and $\underline{\nu} \cdot \underline{\lambda}:=\sum_{k=1}^{n-1} \nu_{k} \lambda_{k}$. Thus, we can find all the series $F_{\underline{\nu}}(q)$ if we know them for all $\underline{\nu}-\frac{\underline{\varepsilon}^{(1)}}{2}$ belonging to a finite subset $M \subset \mathbb{Z}^{n-1}$ given by the intersection of the lattice $\mathbb{Z}^{n-1}$ with a fundamental domain of its sublattice $2 N A^{(n)}\left(\mathbb{Z}^{n-1}\right)$ (here we use the fact that $A^{(n)}$ is a nondegenerate integral matrix). In fact, if we split the sum in (B.6) into two sums, the first, over the fundamental domain $L+\frac{\underline{\varepsilon}^{(1)}}{2}$ and the second, over the its translates $2 N A^{(n)}(\underline{\lambda})$ - we find (using (B.7)):

$$
F\left(\zeta_{12}, \ldots, \zeta_{n-1 n} ; \tau\right)=\sum_{\underline{\nu} \in L+\frac{\underline{\varepsilon}^{(1)}}{2}} F_{\underline{\nu}}(q) F_{\underline{\nu}}^{(N)}\left(\zeta_{12}, \ldots, \zeta_{n-1 n} ; \tau\right)
$$

where the series

$$
\begin{aligned}
& F_{\underline{\nu}}^{(N)}\left(\zeta_{12}, \ldots, \zeta_{n-1} ; \tau\right):= \\
& \quad:=\sum_{\underline{\lambda} \in \mathbb{Z}^{n-1}}(-1)^{\underline{\lambda} \cdot \underline{\varepsilon}^{(2)}} q^{N} \underline{\lambda} \cdot A^{(n)}(\underline{\lambda})-\underline{\nu} \cdot \underline{\lambda} e^{2 \pi i\left(\underline{\nu}+2 N A^{(n)}(\underline{\lambda})\right) \cdot \underline{\zeta}}
\end{aligned}
$$

$\left(\underline{\zeta}:=\left(\zeta_{12}, \ldots, \zeta_{n-1 n}\right)\right)$ are absolutely convergent to analytic functions in $\underline{\zeta}$ and $\tau$ with $\operatorname{Im} \tau>0$ according to the general theory of the theta-series. If $F$ is symmetric as a series in $\zeta_{1}, \ldots, \zeta_{n}$ then the above basic $F^{(N)}$ series can be further symmetrized in $\zeta_{k}(k=1, \ldots, n)$.

This completes the proof of Proposition 3.4.

\section{References}

[1] M.F. Atiyah, I.G. Macdonald, Introduction to Commutative Algebra. AddisonWesley, Reading, Mass., 1969

[2] R.E. Borcherds, Vertex algebras, Kac-Moody algebras, and the Monster, Proc. Nat. Acad. Sci. USA 83 (1986) 3068-3071 
[3] R.E. Borcherds, Vertex algebras. in Topological Field Theory, Primitive Forms and Related Topics (Kyoto, 1996), 35-77, Progr. Math., 160, Birkhäuser Boston, Boston, MA, 1998. q-alg/9706008

[4] H.J. Borchers, Field operators as $C^{\infty}$ functions in spacelike dimensions, Nuovo Cim. 33 (1964) 1600

[5] J. Bros, D. Buchholz, Towards a relativistic KMS condition, Nucl. Phys, B423 (1994) 291-318

[6] D. Buchholz, E. Wichmann, Causal independence and the energy-level density of states in local quantum theory, Commun. Math. Phys. 106 (1986) 321-344

[7] D. Buchholz, C. d'Antoni, R. Longo, Nuclear maps and modular structure II, Commun. Math. Phys. 129 (1990) 115-138

[8] D. Buchholz, On hot bangs and the arrow of time in relativistic quantum field theory, Commun. Math. Phys. 237 (2003) 271-288, hep-th/0301115

[9] P.A.M. Dirac, Wave equations in conformal space, Ann. Math. 37 (1936) 429-442

[10] J.S. Dowker, K. Kirsten, Elliptic functions and temperature inversion on spheres, Nucl. Phys. B638 (2002) 405-432; hep-th/0205029

[11] E. Frenkel, D. Ben-Zvi, Vertex Algebras and Algebraic Curves, AMS, 2001

[12] From Number Theory to Physics, M. Waldschmidt et al. (eds.) Springer 1992, 1995; see, in particular, Chapter 2: J.-B. Bost, Introduction to compact Riemann surfaces, Jacobians, and Abelian varieties, pp. 64-211; Chapter 3: H. Cohen, Elliptic curves, pp. 212-237; Chapter 4: Don Zagier, Introduction to modular forms, pp. 238-291

[13] F. Gursey, S. Orfanidis, Conformal invariance and field theory in two dimensions, Phys. Rev. D7 (1973) 2414-2437

[14] R. Haag, Local Quantum Physics: Fields, Particles, Algebras, Springer-Verlag 1992; 2-nd revised edition, 1996

[15] R. Haag, N. Hugenholtz, M. Winnik, On the equilibrium states in quantum statistical mechanics, Commun. Math. Phys. 5 (1967) 215-136

[16] M. Hortacsu, Explicit examples of conformal invariance, Int. Journ. Theor. Phys. 42 (2003) 49

[17] M. Hortacsu, R. Seiler, B. Schroer, Conformal symmetry and reverberations, Phys. Rev. D5 (1972) 2519-2534

[18] R. Jost, The General Theory of Quantized Fields, Amer. Math. Soc. Publ., Providence R.I. 1965

[19] V.G. Kac, Vertex Algebras for Beginners, AMS, ULS 10, Providence, R.I. 1996, 2nd ed. 1998

[20] Y. Kawahigashi, R. Longo, Classification of two-dimensional conformal nets with $c<1$ and 2-cohomology vanishing for tensor categories, Commun. Math. Phys. 244 (2004) 63-97, math-ph/0304022

[21] H.A. Kramers, G.H. Wannier, Statistics of two-dimensional frerromagnet I, II, Phys. Rev. 60 (1941) 252-262, 263-276

[22] S. Lang, Elliptic Functions, 2nd ed., Graduate Texts in Mathematics 112, Springer, N.Y. 1987

[23] M. Lüscher, G. Mack, Global conformal invariance in quantum field theory, Commun. Math. Phys. 41 (1975) 203-234

[24] G. Mack, All unitary representations of the conformal group $S U(2,2)$ with positive energy, Commun. Math. Phys. 55 (1977) 1-28

[25] D. Mumford, Tata Lectures on Theta I, II, Birkhauser, Boston et al. 1983, 1984

[26] N.M. Nikolov, Vertex algebras in higher dimensions and globally conformal invariant quantum field theory, Commun. Math. Phys. 253 (2005) 283-322, hep-th/0307235

[27] N.M. Nikolov, Ya.S. Stanev, I.T. Todorov, Four dimensional CFT models with ra- 
tional correlation functions, J. Phys. A: Math. Gen. 35 (2002) 2985-3007, hepth/0110230

[28] N.M. Nikolov, Ya.S. Stanev, I.T. Todorov, Globally conformal invariant gauge field theory with rational correlation functions, Nucl. Phys. B 670 [FS] (2003) 373-400, hep-th/0305200

[29] N.M. Nikolov, I.T. Todorov, Rationality of conformally invariant local correlation functions on compactified Minkowski space, Commun. Math. Phys. 218 (2001) 417436, hep-th/0009004

[30] N.M. Nikolov, I.T. Todorov, Conformal quantum field theory in two and four dimensions, in: Proceedings of the Summer School in Modern Mathematical Physics, eds. B. Dragovich, B. Sazdović, Belgrade 2002, pp. 1-49

[31] B. Schroer, Braided structure in 4-dimensional quantum field theory, Phys. Lett. B506 (2001) 337-343, hep-th/0012021

[32] I.E. Segal, Causally oriented manifolds and groups, Bull. Amer. Math. Soc. 77 (1971) 958-959

[33] I.E. Segal, Covariant chronogeometry and extreme distances. III Macro-micro relations, Int. J. Theor. Phys. 21 (1982) 851-869

[34] R.F. Streater, A.S. Wightman, PCT, Spin and Statistics, and All That, Princeton Univ. Press, Princeton, N.J. 2000

[35] I.T. Todorov, Infinite dimensional Lie algebras in conformal QFT models, in: A.O. Barut, H.-D. Doebner, (eds.) Conformal Groups and Related Symmetries. Physical Results and Mathematical Background, Lecture Notes in Physics 261, pp. 387-443, Springer, Berlin et al. 1986

[36] A. Uhlmann, Remarks on the future tube, Acta Phys. Pol. 24 (1963) 293; The closure of Minkowski space. ibid. pp. 295-296.

[37] M. Yoshida, Hyprgeometric Functions, My Love, Vieweg, Braunshweig/Wiesbaden 1997 (see, in particular, Chapter II. Elliptic curves, pp. 29-59)

[38] Y. Zhu, Modular invariance of characters of vertex operator algebras, J. Amer. Math. Soc. 9:1 (1996) 237-302 